
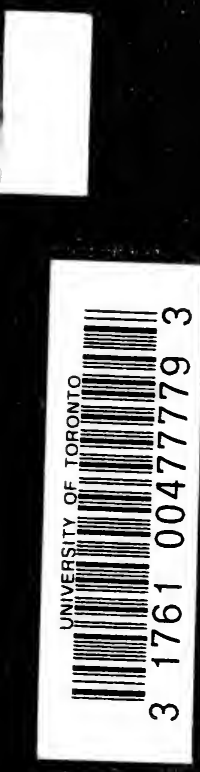
Digitized by the Internet Archive in 2007 with funding from Microsoft Corporation 


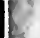

$E^{2}$

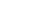

$$
\text { . }
$$



NEW MATHEMATICAL PASTIMES 


\section{CAMBRIDGE UNIVERSITY PRESS}

C. F. Clay, Manager

LONDON : FETTER LANE, E.C. 4

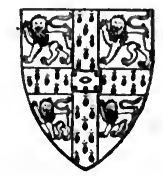

NEW YORK : THE MACMILLAN CO. BOMBAY

CALCUTTA MACMILLAN AND CO., LTD. MADRAS )

TORONTO : THE MACMILLAN CO. OF CANADA, LTD.

TOKYO : MARUZEN-KABUSHIKI-KAISHA

ALI. RIGHTS RESFRVEI) 


\title{
NEW \\ MATHEMATICAL PASTIMES
}

\author{
BY \\ Major P. A. MACMAHON, R.A. \\ D.Sc., Sc.D., LL.D., F.R.S. \\ ST JOHN'S COLLEGE, CAMBRIDGE
}

CAMBRIDGE

AT THE UNIVERSITY PRESS

I 92 I 


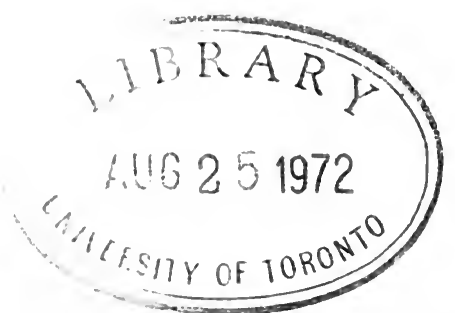

...; he put together a piece of joinery, so crossly indented and whimsically dove-tailed; a cabinet so variously inlaid; such a piece of diversified mosaic ; such a tessellated pavement without cement ; ...

Edmund Burke, 1774, on American Taxation.

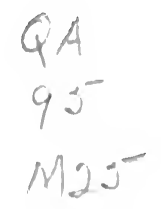




\section{PREFACE}

EDwards. You are a philosopher, Dr Johnson. I have tried too in my time to be a philosopher, but I don't know how, cheerfulness was always breaking in.

BOSWELL'S Life of Johnson.

THE author of this book has, of recent years, devoted much 1 time and thought to the development of the subject of 'Permutations and Combinations' with which all students are familiar. He has been led, during that time, to construct, for use in the home circle, various sets of pieces, of elementary geometrical shapes based upon these ideas, and he now for the first time brings them together with the object of introducing, in a wider sphere, what he believes to be a pleasant by-path of mathematics which has almost entirely escaped the attention of the well-known writers upon Mathematical Recreations and Amusements. The book differs in toto from their works because everything that it contains, with scarcely an exception, is the invention of the author.

It is not a bringing together of materials derived from wholly different ideas. From beginning to end it proceeds along one defined path from which it never diverges. One continuous thread of thought runs through it from cover to cover.

Indeed, in view of the excellent collections of mathematical recreations that have proceeded from the pens of Ed. Lucas, W. W. Rouse Ball, W. Ahrens, H. E. Dudeney and others it would appear that there is no room for another book upon the lines upon which these have been written. I would make particular reference to the work in two volumes of W. Ahrens of Magdeburg and draw attention to the Bibliography which it contains. It involves nearly eight hundred titles. This must have necessitated much research. He has included a large number of works upon magic squares and upon the knight's tour and other chess-board amusements. These have been omitted from the short list appended to this book, which had been almost completed when the list of Ahrens came into view. Many of the books appear to be rare, as they could not be found in the library of the British Museum. 
Part I deals with sets of pieces of exactly the same size and shape, but differently coloured, numbered or otherwise distinguished upon combinatory principles in such wise that no two pieces of a set are identical. It is shewn that such sets lend themselves to a great variety of pastimes, and the reader who will take the trouble to construct sets and employ suitable colours will find that he is truly in a kaleidoscope of constantly changing colour effect, the attractiveness of which will fully repay him for his trouble. The designs obtained from some of the contact systems frequently possess beautiful symmetry.

It has not been found possible to produce the book in colour, and as the author has himself invariably investigated the sets in colours he must confess to a feeling of disappointment at the appearance of the pages.

In colours fresh, originally bright,

Preserve its portrait and report its fate:

The Complaint.

Briefly, Part I may be described as generalised dominoes.

Part II follows Part I in a natural manner, for it is a mere transformation of it. The sets of Part I have the same shape but are differently coloured. Those of Part II have the same colours but are differently shaped. The transformations can be carried out always in an infinite number of ways and give ample scope for taste and ingenuity. One merely requires squared paper (in the millimetre unit by preference), ruler and compasses to be able to design and construct a means of endless amusement.

How rich the prospect! and for ever new!

And newest to the man who views it most;

For newer still in infinite succeeds.

The Consolation.

Part III follows quite naturally upon Part II because the schemes of transformation prove, after a little consideration, to be a comprehensive method of designing repeating patterns for decorative work. Here we have pieces of the same size and shape which can be employed to completely cover a pavement or other flat surface. The only repeating patterns that were known in early times appear to have been the equilateral triangle, square and regular hexagon, the only regular polygons which possess the 'repeating' property. Great advances were made in 
the middle ages by the Arabian and Moorish architects, and in many of their buildings-ex. gr. the Alhambra at Granadaelaborate repeating patterns, based upon the square and its derivatives, are in evidence and are most effective. Each of these can be labelled with base and contact system in the classification in this book. Repeating patterns are in constant view in the home in parquet floors, carpets, paper-hangings, apparel, woven fabrics, etc.

An attempt has been made to interest the reader while drawing as little as may be upon his knowledge of geometry. If he will study Part III after having become familiar with the transformations of Part II he will be able to make designs for home work to his heart's content.

Beauty of form as depending upon symmetry of some kind is brought forward as being necessarily an important object of the designer. An elementary discussion of this precedes definite rules for obtaining symmetry.

The fact that the number of different patterns is unlimited, in each of the categories, leaves much to the judgment and fancy of the designer who can give free play to his imagination.

The patterns usually met with in public edifices and private homes are on hard and fast lines, shewing curiously little variety and cleverness in view of the fact that the theme is one of infinite scope.

The subject may be regarded, it is thought, as an important recreation because the construction of the designs and assemblages possesses a distinct fascination.

What we admire we praise; and when we praise

Advance it into notice, that its worth

Acknowledg'd, others may admire it too.

The Tusk.

The subject of Part III has been carried much further than appears in these recreations, by the author and others. A work, entirely devoted to it, is in hand and may shortly appear.

P. A. M.

September, I921. 


\section{TABLE OF CONTENTS}

\section{PART I}

\section{PASTIMES BASED UPON SIMPLE GEOMETRICAL FORMS}

ART.

I Preliminary observations upon dominoes .

PAGE

2-10 Equilateral Triangle Pastimes. Set of 24 pieces with 4 colours repeatable. The different contact systems. The set of ro pieces. The set of 13 pieces.

I I-I 3 The set of 20 triangles involving 5 colours not repeatable. The contact systems and boundary types. . . . . . 16

14-15 The set of 12 which involves a particular colour . . . 20

I6 The set of 8 which involves 4 colours . . . . . 22

17-19 Square Pastimes. The set of 24 which involves 3 colours repeatable . . . . . . . . . . . 23

20-22 The set of 20 which is only symmetrical in 2 colours . . 26

23-25 A set of 16 symmetrical in 2 colours . . . . . . 28

26-27 A set of 15 . . . . . . . . . . . . . 33

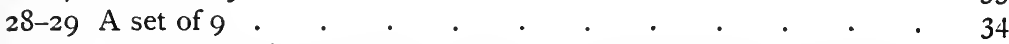

30 A set of 24 involving $;$ colours not repeatable but having a particular colour upon each piece . . . . . . . 36

3 I Varieties of boundary types . . . . . . . . 38

32-33 Right-angled Triangle Pastimes. A set of 24 involving 4 colours. Types of internal structure . $\quad . \quad \cdot \quad \cdot \quad \cdot \quad \cdot \quad \cdot 39$

34-35 A Cube Pastime. $\Lambda$ set of 30 involving 6 colours not repeatable. A set of 8 which is 'contained' in any one piece. Reciprocity. Analogy with the Triangle and Square . . . . .

36 Regular Hexagon Pastimes. A set of 24 involving 6 colours not repeatable but every piece shewing one particular colour. Three figures of assemblage . . . . . . .

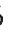
2 3

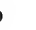
3 6 8

\section{PART II}

\section{THE TRANSFORMATION OF PART I}

37 The general idea of transformation explained. The two natures of transformation . . . . . . . . .

38-45 The Equilateral Triangle. Transformation of the Pastimes nos. 2-IO of Part I with diagrams . . . . . . . 53

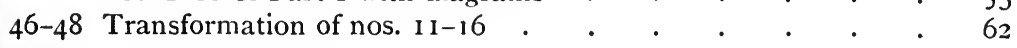

49 Alteration of compartment boundaries that must precede the transformation of the Right-angled Triangle Pastimes . . . 
50-54 The Square. Transformations of nos. 17-22 . . . . 68

55-57 Transformations of nos. 23-25 with a large number of specimen boundaries

58 A transformation of no. 30 with diagrams. $. \quad . \quad$.

\section{PART III}

\section{THE DESIGN OF 'REPEATING PATTERNS' FOR DECORATIVE WORK}

59 General ideas shewing how the subject arises naturally from Parts I and II . . . . . . . . . . . 80

60-64 Elementary notions concerning patterns . . . . .

8 I

65 Removal of restrictions upon design . . . . . . .

66-69 The Triangle Base. The two contact systems and methods of assembling the patterns. Principles upon which symmetry in the patterns can be secured. Specimen patterns and assemblages. The 'Helmet' pattern. 'Aspects'.

84

70-7I The Square Base. The category that arises from the first contact system with numerous examples. Assemblages shewing different numbers of aspects for the same pattern

91

72-73 Further principles which enable symmetry to be secured

74-75 A second contact system with examples of patterns and assemblages . . . . . . . . . . . 96

76-78 Third and fourth contact systems with examples . . . 98

79-80 Fifth contact system. Remarks upon symmetry. The equilateral pentagon pattern . . . . . . . . 100

81 Special study of the equilateral pentagon . . . . . 102

82-84 The Regular Hexagon Base. The two contact systems with remarks upon symmetry and numerous examples . . . 106

$8 ;$ Concluding general remarks. The evolution of the pattern, which is a combination of the regular octagon and square, from the square base. The colouring of assemblages of repeating patterns .

S6 The construction of the various pastimes . . . . . 


\section{PART I}

\section{PASTIMES BASED UPON SIMPLE GEOMETRICAL FORMS}

Come track with me this little vagrant rill, Wandering its wild course from the mountain's breast.

Doubleday.

1. The amusements to which this book is devoted are played with a number of cards or pieces which involve or are based upon certain regular or other polygons which are distinguished upon the sides with certain colours or numbers, in such wise that for a given number of colours and for given conditions of their occurrence there is one piece for every possible arrangement of the colours upon the sides.

The principal existing amusement which embodies this idea

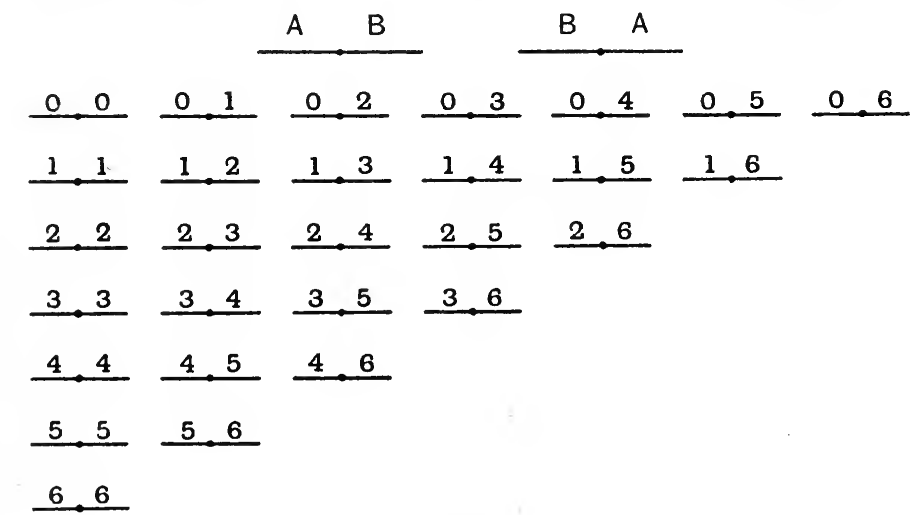

Fig. I.

is the game of dominoes which in various varieties is known all over the world.

The pieces here consist essentially of straight lines-each piece being a line as $A B$ in fig. I.

In the most common set seven numbers are employed

$$
\text { o, I, 2, 3, 4, 5, } 6
$$

and these are arranged in every possible way upon the two M. P. 
halves $A, B$ in such wise that the pieces $A-B$ and $B-A$ (fig. I) are regarded as identical.

We thus obtain 28 pieces as numbered in fig. I.

For convenience, in actual practice, the pieces are broadened so as to consist of two equal squares joined about a side, each square being devoted to a number.

Dudeney, Lucas and others have shewn that, apart from the different games, Draw, Matadore, Cyprus, etc., the pieces may be placed together so as to fulfil certain conditions and thus lend themselves to a great variety of patiences and puzzles.

Other sets of dominoes have been popular at different times and in different places; such for instance as employ ten or thirteen different numbers so as to proceed to the double-nine and double-twelve pieces respectively.

\section{EQUILATERAL TRIANGLE PAS'TIMES}

A scheme, analogy pronounced so true:

Analogy, man's surest guide below.

The Complaint.
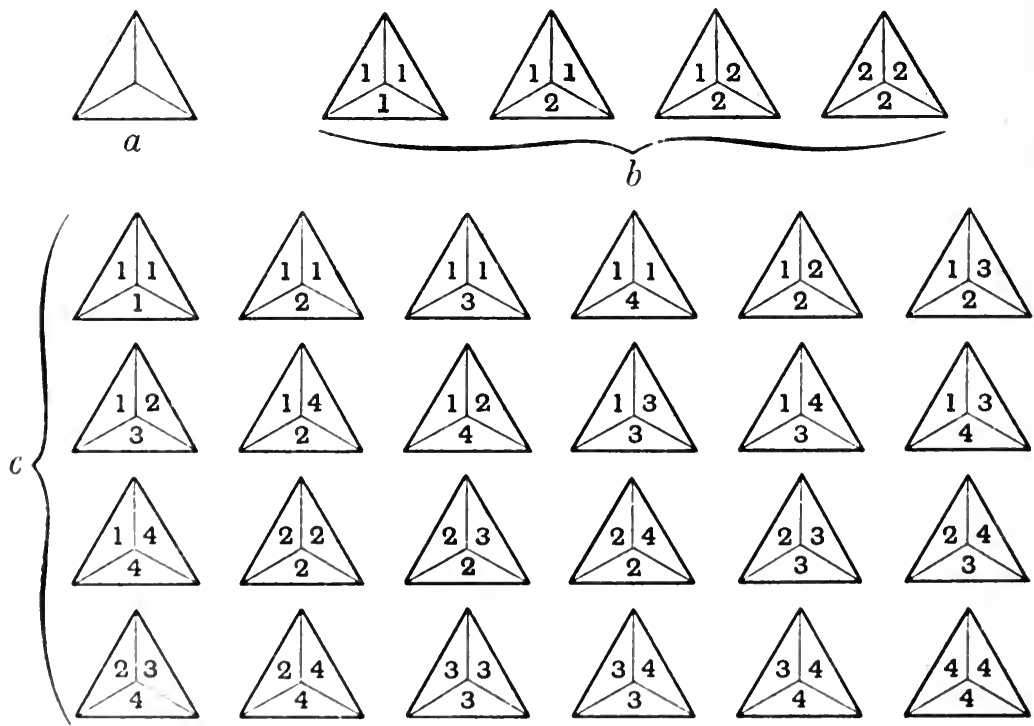

Fig. 2.

2. In order to develop this principle take an equilateral triangle (fig. $2 a$ ) and join the angular points to the centre of the 
circumscribing circle. This point is also the point of intersection of the three perpendiculars from the angular points upon the opposite sides and each perpendicular has the point as one of its points of trisection. The construction divides the triangle into three equal and similar parts. These parts we will call compartments of the triangle.

If we employ two colours, denoting them by the numbers I, 2 and, as in dominoes, allow repetitions of the same numbers on the same triangle we obtain the four pieces of fig. $2 b$.

Three colours yield eleven and four colours twenty-four pieces*. The twenty-four pieces are set forth arranged in a convenient order in fig. $2 c$.

* The problem of enumerating the number of ways of colouring the sides of a regular polygon of $m$ sides with $n$ colours, repetitions of colour on the sides of the polygon being permitted, may be studied by means of the Theory of Cyclical Permutations which has been very clearly set forth by Netto in his Combinatorik (Leipzig, Verlag von B. G. Teubner, 1901). If upon a set of polygons the $n$ colours are repeated $\alpha, \beta, \gamma, \ldots$ times respectively, where any of the numbers $\alpha, \beta, \gamma, \ldots$ may be zero and $\alpha, \beta, \gamma, \ldots$ do not contain any common factor greater than unity, the number of different polygons of the set is

$$
\frac{(n-1) !}{a ! \beta ! \gamma ! \ldots},(n ! \text { means the factorial of } n \text {, sometimes written } \underline{n})
$$

but if the numbers $\alpha, \beta, \gamma, \ldots$ involve a common factor $d$, where $d$ is a prime number, and $\alpha=d \alpha_{1}, \beta=d \beta_{1}, \gamma=d \gamma_{1}, \ldots n=d n_{1}$, the number of different polygons of the set is

$$
\frac{(n-1) !}{a ! \beta ! \gamma ! \ldots}+\frac{n-n_{1}}{n} \cdot \frac{\left(n_{1}-1\right) !}{\alpha_{1} ! \beta_{1} ! \gamma_{1} ! \ldots} \text {. }
$$

The results are more complicated when the common divisor $d$ is a composite number but are given (l.c.).

Utilising these results we find that the enumerations are for

$$
\begin{aligned}
& \text { Triangle } \frac{1}{3} n\left(n^{2}+2\right), \\
& \text { Square } \frac{1}{4} n(n+1)\left(n^{2}-n+2\right), \\
& \text { Pentagon } \frac{1}{5} n\left(n^{4}+4\right), \\
& \text { Hexagon } \frac{1}{6} n(n+1)\left(n^{4}-n^{3}+n^{2}+2\right), \\
& \text { Heptagon } \frac{1}{7} n\left(n^{6}+6\right) .
\end{aligned}
$$

The mathematical reader will be able to establish that when the polygon has $p$ sides, $p$ being a prime number, the enumeration is given by

$$
\frac{\mathrm{I}}{p} n\left(n^{p-1}+p-\mathrm{I}\right) \text {. }
$$

When repetitions of colour are not permitted the enumeration, in the case of a regular polygon of $m$ sides, is

$$
\frac{\mathrm{I}}{m} \frac{n !}{(n-m) !} \text {. }
$$

If one particular colour is to occur upon each polygon the enumeration is given by

$$
\frac{(n-1) !}{(n-m) !} \text {. }
$$


These may be set up into a regular hexagon as in fig. 3, a circumstance which supplies a useful starting-point for the study of the system of triangles.

IVhen we assemble the pieces so as to form the hexagon we may adopt some principle of contact between the compartments of different triangles. The most obvious one is to insist that a

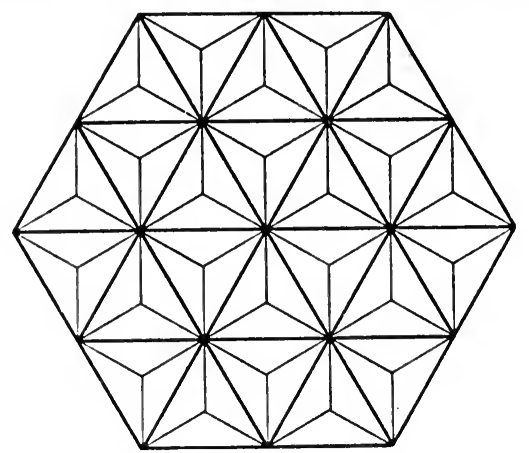

Fig. 3.

compartment shall lie adjacent to a compartment similarly numbered or coloured. With this single condition the number of arrangements is so large that it proves to be possible to impose other conditions affecting the compartments which lie upon the exterior boundary of the hexagon.

Meditation here

May think down hours to moments.

The Tisk.

In order to realise what may be the nature of these boundary conditions we must observe that since each of the 24 triangles possesses 3 compartments there are altogether 72 compartments. The four colours are symmetrically involved so that each must appear upon one-fourth of 72 or upon 18 compartments. By reason of the specified contact condition a particular colour must occur an even number of times inside the hexagon and since 18 is an even number it follows that a particular colour must occur an even number of times upon the boundary. We see that there are 12 boundary compartments so that any particular colour must occur upon the boundary a number of times denoted by one of the even numbers

$$
\mathrm{O}, 2,4,6,8,10, \mathrm{I} 2 .
$$


Thence it follows that, considering all four colours, the occurrence upon the boundary must be according to one of the eight schemes:

Colours

\begin{tabular}{r|r|r|r} 
I & 2 & 3 & 4 \\
\hline I2 & 0 & 0 & 0 \\
IO & 2 & 0 & 0 \\
8 & 4 & 0 & 0 \\
8 & 2 & 2 & 0
\end{tabular}

\begin{tabular}{l|l|l|l} 
I & 2 & 3 & 4 \\
\hline 6 & 4 & 2 & 0 \\
6 & 2 & 2 & 2 \\
4 & 4 & 4 & 0 \\
4 & 4 & 2 & 2
\end{tabular}

The fifth of these types indicates that the colours occur upon the boundary 6, 4, 2, o times respectively and the type is looked upon as the same when the colours are merely interchanged; so
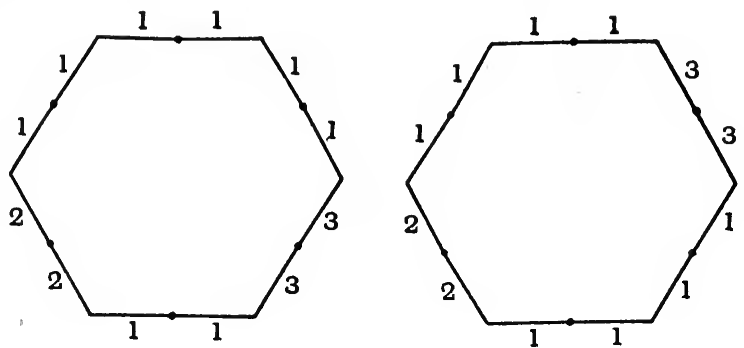

Fig. 4.

that the 24 arrangements of the numbers $6,4,2,0$, are all of the same type.

Each type except the first, in which all the boundary compartments are of the same colour, involves several varieties of
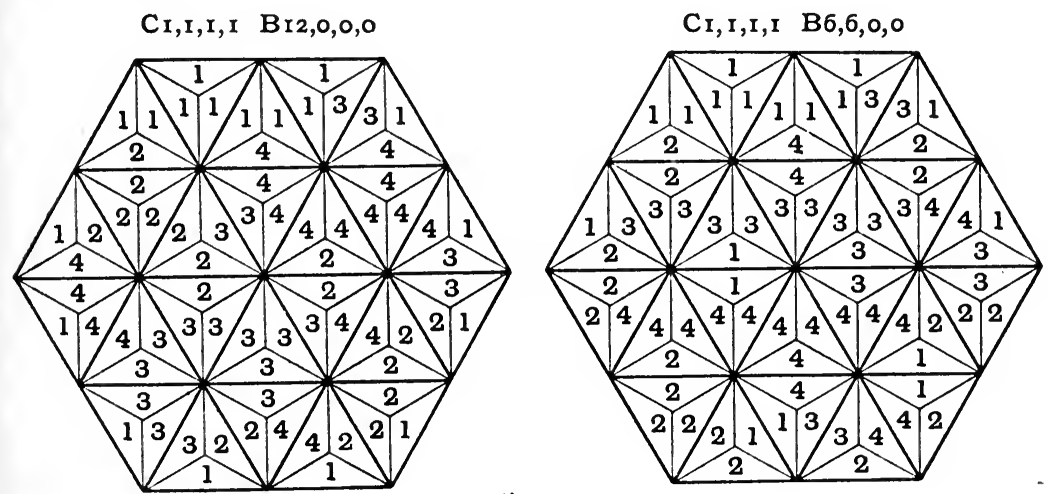

Fig. 5 . 
boundary depending upon the way in which the given boundary colours are arranged.

Thus for the fourth type two varieties are as in fig. 4 .

With the stated condition of contact inside the hexagon each of the eight types can be actually set up and many varieties of types. Some of these are difficult to obtain and make considerable demands upon the skill and patience. It is not known if any of the varieties are in reality impossible boundary conditions.

Two examples are given in fig. 5 .

They are framed upon what we will call the First Contact

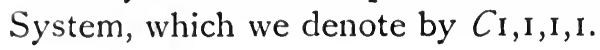

So from the first eternal order ran,

And creature link'd to creature, man to man....

The link dissolves, each seeks a fresh embrace, Another love succeeds, another race.

Essay on Man.

3. We may adopt other contact conditions inside the hexagon. We have four colours at disposal, I, 2, 3, 4 suppose.

In the first contact sy'stem we have

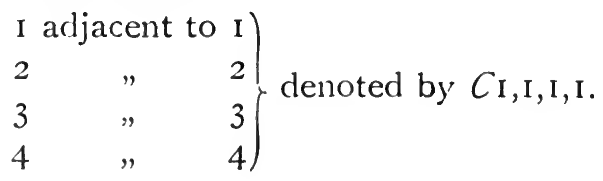

We take as our second contact system

$$
\left.\begin{array}{lll}
\text { I } & \text { adjacent to } & \text { I } \\
2 & \# & 2 \\
3 & " & 4
\end{array}\right\} \text { denoted by } C \text { I, I, 2, }
$$

so that one pair of 3 and 4 compartments lie as in fig. 6 .

As regards the colours I, 2, we know, from what has been said above, that each must appear an even number of times upon the boundary. The third condition necessitates the colours 3,4 appearing an equal number of times inside the hexagon. It follows that each must appear the same number of times upon the boundary, but this number may be even or uneven.

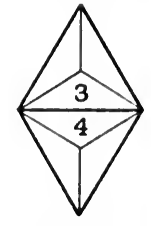

Fig. 6 . 
The possible types of boundary are now 16 in number:

\begin{tabular}{|c|c|c|c|c|}
\hline Colours & I & 2 & 3 & 4 \\
\hline & I2 & $\mathrm{O}$ & 0 & 0 \\
\hline & 10 & 2 & o & 0 \\
\hline & 8 & 4 & $\mathrm{O}$ & 0 \\
\hline & 6 & 6 & 0 & 0 \\
\hline & 10 & 0 & I & I \\
\hline & 8 & 2 & I & I \\
\hline & 6 & 4 & I & I \\
\hline & 8 & 0 & 2 & 2 \\
\hline
\end{tabular}

\begin{tabular}{l|l|l|l}
$\mathbf{I}$ & 2 & 3 & 4 \\
\hline 6 & 2 & 2 & 2 \\
4 & 4 & 2 & 2 \\
6 & 0 & 3 & 3 \\
4 & 2 & 3 & 3 \\
4 & 0 & 4 & 4 \\
2 & 2 & 4 & 4 \\
2 & 0 & 5 & 5 \\
0 & 0 & 6 & 6
\end{tabular}

and there are varieties of every type except the first.

Most of these types and many varieties have been actually set up. Probably every type is possible but nothing is known
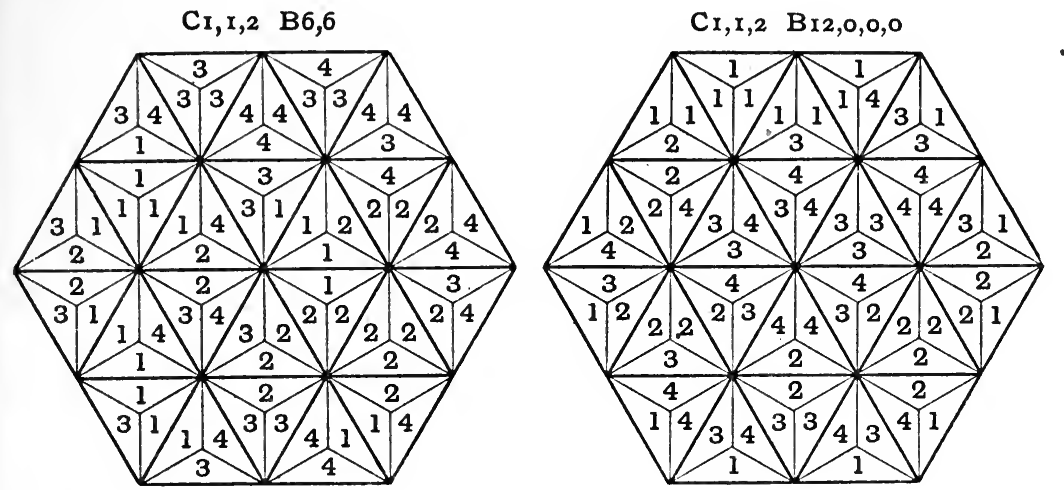

Fig. 7 .

concerning the possibility of the numerous varieties except that it is certain that a great number of these can be arranged.

Two examples are given in fig. 7 .

Arithmetick would erre exceedingly,

Forgetting to divide and multiply;

Geometry would lose the altitude,

The crassie longitude and latitude;

And musick in poore case would be o'er throwne,

But that the goose quill pricks the lessons downe.

TAYLOR's Works, I630.

4. For a third contact system we take

I adjacent to 2

$3 \quad " 4$ 
that is to say the colours I, 3 lie up against the colours 2, 4 respectively. We denote it by $C_{2,2}$.

From what has been said above it is clear that the colours I, 2 must occur the same number of times upon the boundary and also the colours 3,4 .

The types are four in number:

\begin{tabular}{ll|l|l|l} 
Colours & I & $\mathbf{2}$ & 3 & 4 \\
\cline { 2 - 4 } & 6 & 6 & $\mathrm{O}$ & $\mathrm{O}$ \\
5 & 5 & $\mathrm{I}$ & $\mathrm{I}$ \\
& 4 & 4 & 2 & 2 \\
3 & 3 & 3 & 3
\end{tabular}

and every type possesses varieties.
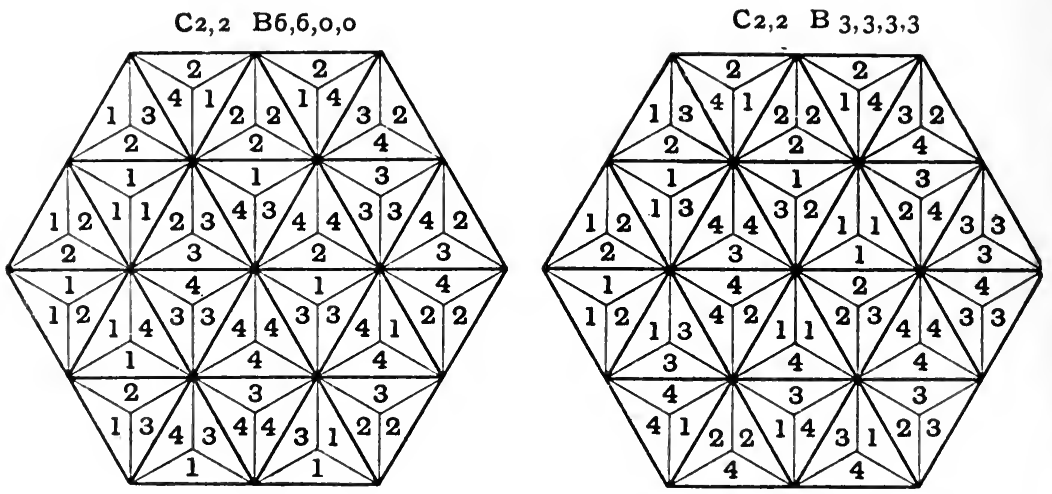

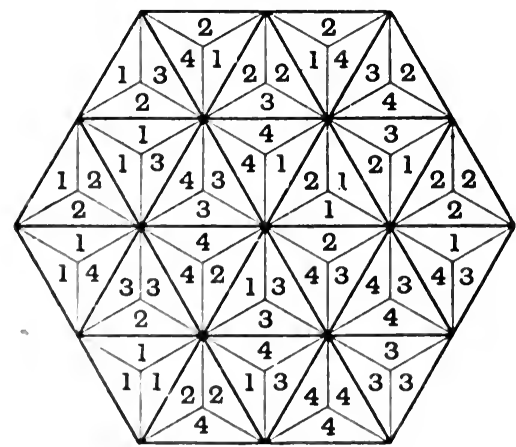

$\mathrm{C}_{2,2} \mathrm{~B}_{4,4,2,2}$

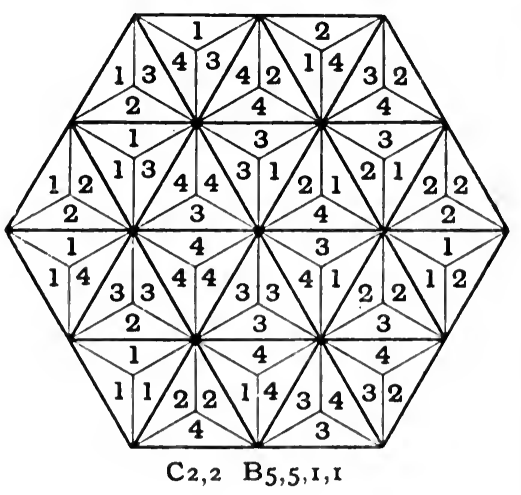

Fig. 8. 
It will be noticed that since the triangles are symmetrical in the four colours, $2,2 \mid 4,4$ is not regarded as a type distinct from $4,4 \mid 2,2$.

These types and many varieties can be set up.

As to the possibility of particular varieties the usual doubt remains.

A number of examples are given in fig. 8 .

5. We have thus, in the case of four colours, three systems of conditions of inside contact.

It is convenient to denote these by

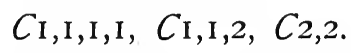

For any number of colours a system is determined by the number of pairs of colours that are associated. The number of systems is the number of ways in which the number, which expresses how many colours are in use, can be made up of ones and twos.

For

$$
\text { I, 2, 3, 4, 5, 6, 7, 8, 9, 10, ... colours }
$$

the systems are $1,2,2,3,3,4,4,5,5,6, \ldots$ in number.

In other words, to obtain the number of systems we add I or 2 to the number of colours, according as that number is uneven or even, and then divide by 2 .

The scheme of triangles that has just been studied may be realised also by taking instead of four colours the blank and one, two, three pips upon the compartments. It then constitutes a
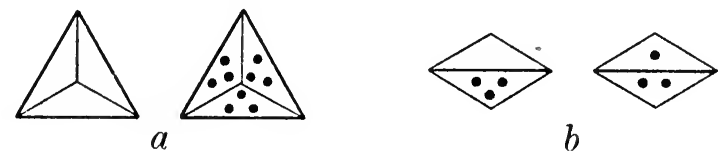

$b$

Fig. 9.

set of triangular dominoes proceeding from treble blank to treble three as in fig. $9 a$.

The first contact system is similar to that adopted for the ordinary game of dominoes, whilst the third system is of the nature of that obtaining in the game of Matadore.

In fact for the third system we may take, as in fig. $9 b$,

blank compartment adjacent to three-pip, one-pip " $\quad$ two-pip, 
so that the condition has the statement that the sum of the pips in adjacent compartments must be three.

Good mother, how shall we find a pig if we do not look about for it? Will it run off o' the spit, into our mouths, think you as in Lubberland, and cry we we?

B. Jonson, Barth. Fair, 1II. 2.

6. We can select, from the complete set of 24 , less numerous sets which are interesting to examine.

There is a set of I I triangles which involves only three specified colours as in fig. IO, but this set cannot be assembled into any interesting shape; so that we attempt to frame an additional condition with the object of reducing the number of triangles to IO.

It will be noticed that Io out of the set of I I are altered by the interchange of the colours I and 2. The triangle which is
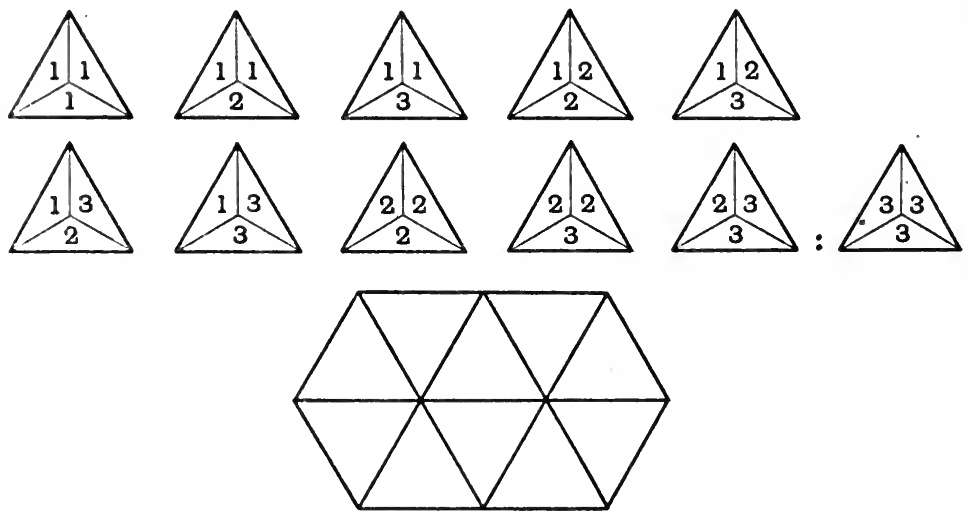

Fig. I0.

not altered by the interchange is the one which has all three compartments with the colour 3 .

We therefore make a new condition to the effect that every triangle is to be altered by the interchange of the colours I, 2 .

We thus obtain ro pieces which can be put together into the hexagonal shape in the lower part of fig. Io.

It is important to bear in mind that this set is not symmetrical in three colours, but notwithstanding that there is only symmetry in regard to the colours 1,2 the set is interesting, as will appear. 
Examination of the Io pieces shews that colours I, 2, 3 occur upon II, I I, 8 compartments respectively.

There are eight boundary compartments.

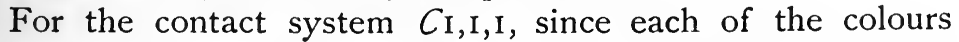
must occur an even number of times inside, it follows that upon the boundary the colours $\mathrm{r}, 2,3$ must occur numbers of times which are uneven, uneven, even respectively.

We obtain the types, six in number:

Colours

\begin{tabular}{l|l|l}
$\mathbf{I}$ & $\mathbf{2}$ & $\mathbf{3}$ \\
\hline 7 & $\mathrm{I}$ & $\mathrm{O}$ \\
5 & 3 & 0 \\
5 & $\mathrm{I}$ & 2
\end{tabular}

\begin{tabular}{l|l|l}
$\mathbf{I}$ & $\mathbf{2}$ & $\mathbf{3}$ \\
\hline 3 & 3 & 2 \\
3 & $\mathbf{I}$ & 4 \\
$\mathbf{I}$ & $\mathbf{I}$ & 6
\end{tabular}

and varieties of each type.

Examples are given in fig. I I.
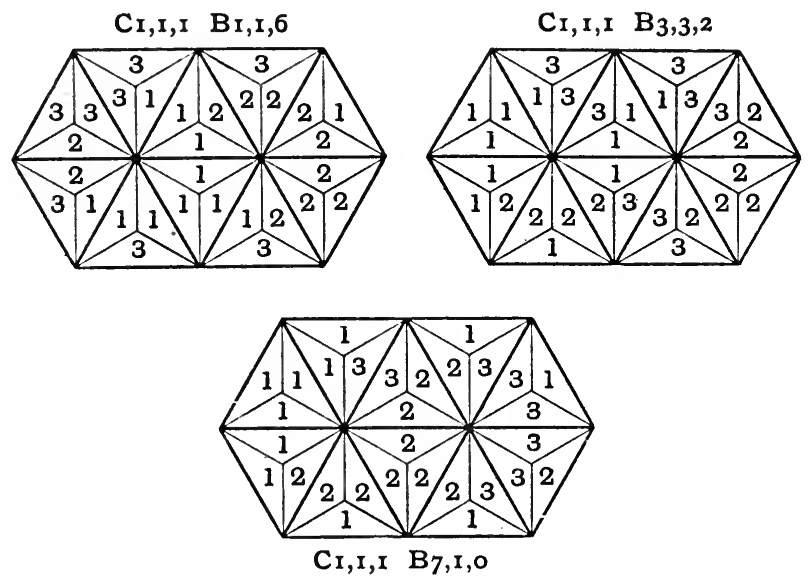

Fig. I I.

The contact system in which $\mathrm{I}$ is adjacent to 2 and 3 adjacent to 3 is different from that in which $\mathrm{I}$ is adjacent to $\mathrm{I}$ and 2 to 3 because of the want of symmetry in these colours. We will denote these by $C_{2,1}$ and $C_{1,2}$ respectively.

7. In the case of $C_{2}, \mathrm{I}$ the colours I, 2 must occur an equal number of times on the boundary and the colour 3 an even number of times not exceeding 6 . The limit 6 is necessary because only 6 of the ro pieces involve the colour 3 . 
We have now four types:

and varieties as usual.

\begin{tabular}{lll:l} 
Colours & I & $\mathbf{2}$ & $\mathbf{3}$ \\
\cline { 2 - 4 } & 4 & 4 & 0 \\
& 3 & 3 & 2 \\
& 2 & 2 & 4 \\
& I & I & 6
\end{tabular}

Examples are given in fig. 12.

An example of every type is given as an indication that probably in other pastimes all types are possible. The reader however will notice that other considerations may rule out certain types, as in fact is seen to be the case in this particular pastime.
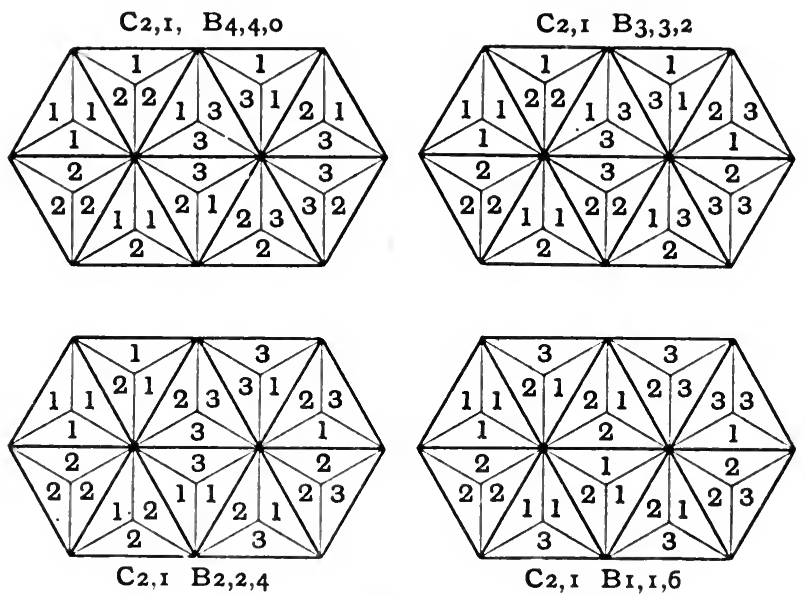

Fig. 12.

8. In the case of the remaining contact system $C_{1,2}$, vi\%. $I$ to $I$ and 2 to 3 , we find colour $\mathrm{I}$ must occur an uneven number of times upon the boundary while colour 2 , since it occurs inside just as often as colour 3 , must occur three more times on the boundary than colour 3 .

Three types arise:

\begin{tabular}{ll|ll} 
Colours & I & $\mathbf{2}$ & 3 \\
& 1 & 5 & 2 \\
& 3 & 4 & 1 \\
& 5 & 3 & 0
\end{tabular}

Examples are given in fig. 13 . 
Here we see that more than one variety of the type $B 5,3,0$ can be arranged.
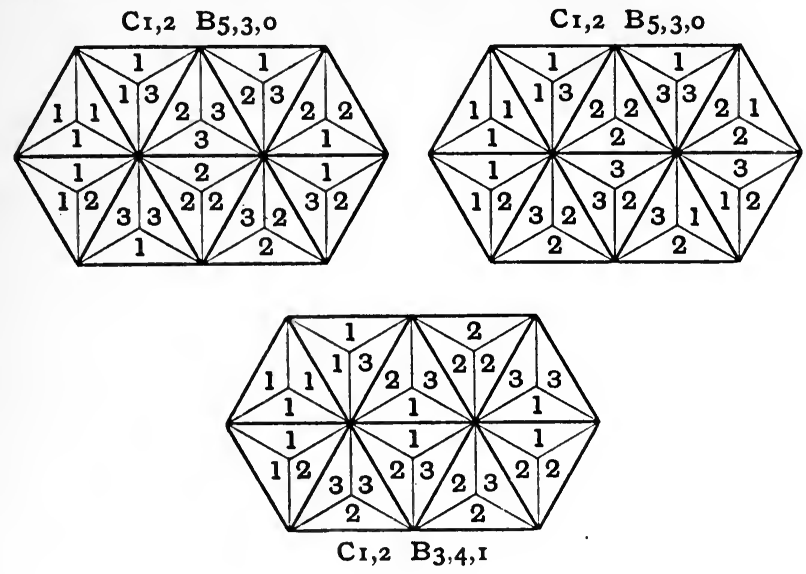

Fig. I3.

9. From the complete set of 24 triangles we now isolate the set containing all of those which involve one particular colour, say the colour 4. There are thirteen as in fig. I4 which can be
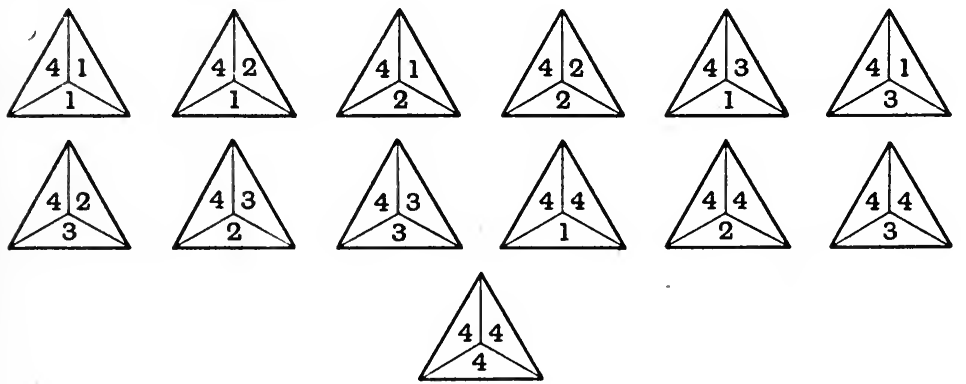

Fig. 14 .

assembled into the form of a semi-regular hexagon or blunted triangle as in fig. I 5.

Observe that the set is symmetrical in the three colours I, 2, 3 but that it is not symmetrical in four colours.

There are nine boundary compartments.

The colours $\mathrm{I}, 2,3,4$ occur in $7,7,7,18$ compartments respectively.

For the contact system $C_{\mathrm{I}, \mathrm{I}, \mathrm{I}, \mathrm{I}}$ the boundary conditions 
are that the colours I, 2, 3 must each occur an uneven $n$ umber of times and 4 an even number.

- There are thus six types:

\begin{tabular}{cc|c|c|c} 
Colours & I & 2 & 3 & 4 \\
\cline { 2 - 4 } & I & I & I & 6 \\
3 & I & I & 4 \\
3 & 3 & I & 2
\end{tabular}

\begin{tabular}{l|l|l|l}
$\mathrm{I}$ & 2 & 3 & 4 \\
\hline 5 & $\mathrm{I}$ & $\mathrm{I}$ & 2 \\
5 & 3 & $\mathrm{I}$ & $\mathrm{O}$ \\
3 & 3 & 3 & 0
\end{tabular}

and the usual varieties.

The type 7, I, I, O is seen at a glance to be impossible.

Examples are given in fig. I 5 .
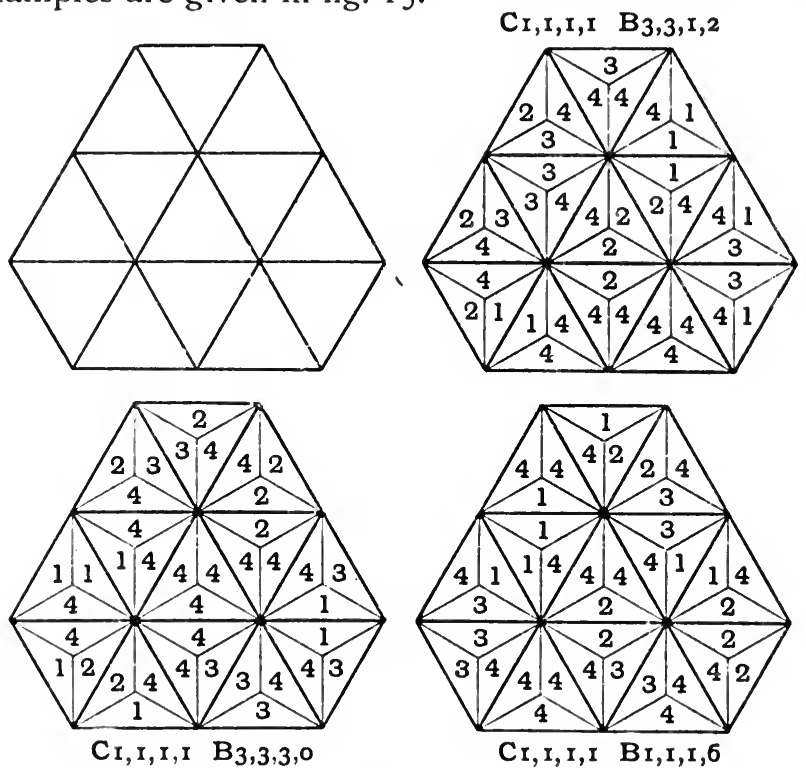

Fig. I5.

To guide my course aright

What mound or steady mere is offered to my sight?

Drayton, Polyolb. I. p. 569 .

10. For the contact system $C_{1,1,2, \text { viz. : }}$

$$
\begin{aligned}
& \text { I to I, } \\
& 2,2, \\
& 3,4,
\end{aligned}
$$

we find as one boundary condition that the colour 4 must occur on the boundary I I more times than colour 3, an impossibility 
since there are only nine boundary compartments. Hence the system $C_{\mathrm{I}, \mathrm{I}, 2}$ is impossible.

For the system $C_{\mathrm{I}, 2, \mathrm{I}}$, viz. :

$$
\begin{aligned}
& \text { I to I, } \\
& 2,3, \\
& 4,4,
\end{aligned}
$$

we find as the boundary conditions

I must occur an uneven number of times,

2 as many times as 3 ,

4 an even number of times,

leading to the types, ten in number:

Colours

\begin{tabular}{l|ll|l} 
I & 2 & 3 & 4 \\
\hline I & I & I & 6 \\
3 & $O$ & 0 & 6 \\
I & 2 & 2 & 4 \\
3 & I & I & 4 \\
I & 3 & 3 & 2
\end{tabular}

\begin{tabular}{l|ll|l}
$\mathbf{I}$ & 2 & 3 & 4 \\
\hline 3 & 2 & 2 & 2 \\
5 & $\mathrm{I}$ & $\mathrm{I}$ & 2 \\
$\mathrm{I}$ & 4 & 4 & $\mathrm{O}$ \\
3 & 3 & 3 & 0 \\
5 & 2 & 2 & $\mathrm{O}$
\end{tabular}

Examples are given in fig. I6.
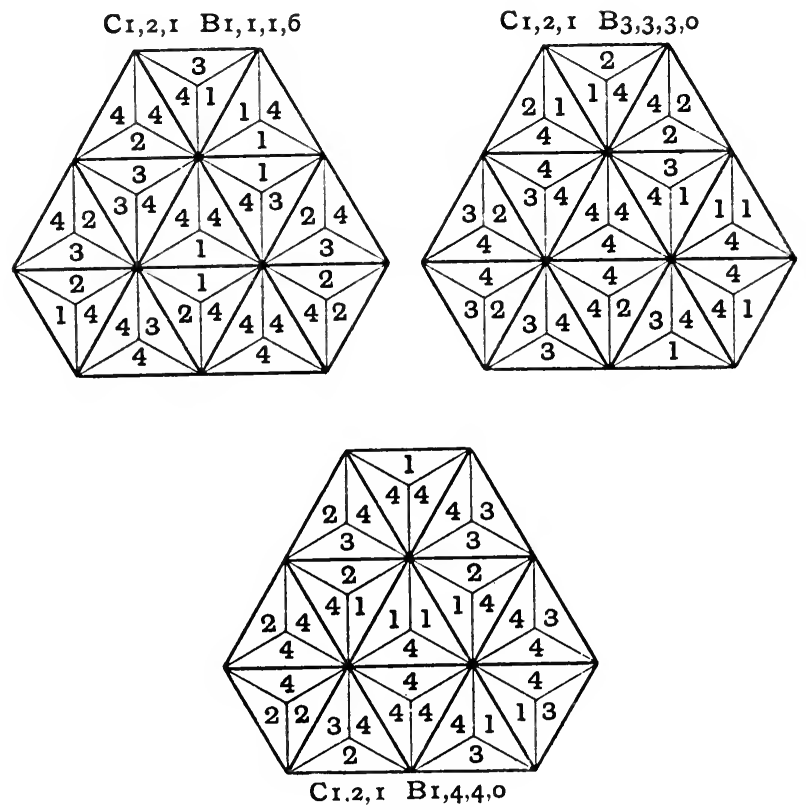

Fig. I6. 
This band dismiss'd, behold another crowd Preferr'd the same request, and lowly bow'd.

The Temple of Fame.

11. The next set of triangles is concerned with the equilateral triangle and five colours, three different colours appearing in the compartments of each triangle. This design leads to the
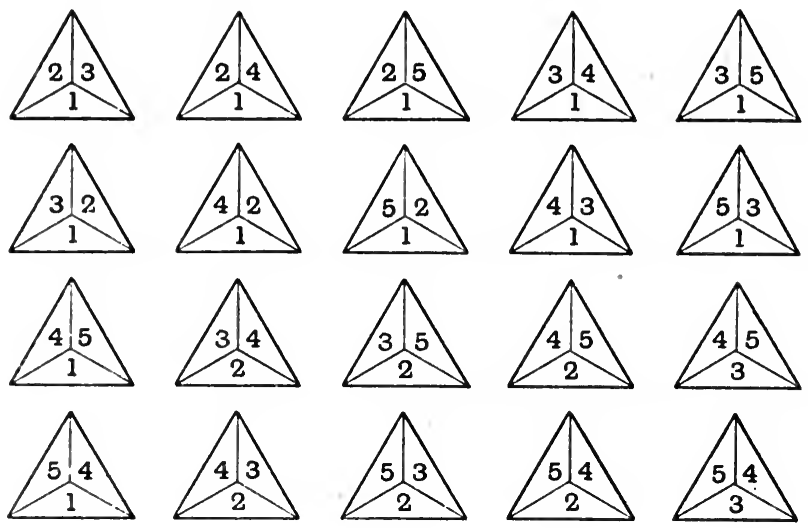

Fig. 17.

twenty triangles, as in fig. 17 , which can be arranged in the semi-regular decagon of fig. I 8 .

There are 12 boundary compartments. Altogether there are

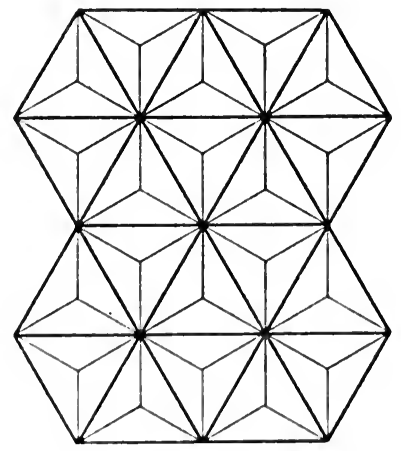

Fig. 18.

6o compartments and 12 of these must belong to each colour as each of the five colours is symmetrically involved. 


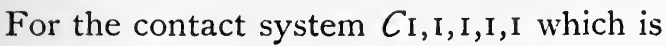

$$
\begin{aligned}
& \text { I to I, } \\
& 2 " 2, \\
& 3 \Rightarrow 3, \\
& 4 ” 4, \\
& 5 \Rightarrow 5,
\end{aligned}
$$

\begin{tabular}{|c|c|c|c|c|c|c|c|c|c|c|}
\hline Colours & $\mathbf{I}$ & 2 & 3 & 4 & 5 & $\mathbf{I}$ & 2 & 3 & 4 & 5 \\
\hline & I2 & 0 & 0 & 0 & 0 & 6 & 4 & 2 & 0 & 0 \\
\hline & 10 & 2 & o & 0 & 0 & 6 & 2 & 2 & 2 & 0 \\
\hline & 8 & 4 & 0 & 0 & 0 & 4 & 4 & 4 & 0 & 0 \\
\hline & 8 & 2 & 2 & 0 & $\mathrm{O}$ & 4 & 4 & 2 & 2 & 0 \\
\hline & 6 & 6 & 0 & 0 & 0 & 4 & 2 & 2 & 2 & 2 \\
\hline
\end{tabular}

we gather that each colour must occur an even number of times upon the boundary.

We have therefore the types, ten in number:

with varieties of each type except the first.

The existence of the first type is somewhat remarkable as there are only just sufficient compartments of a particular colour to go completely round the boundary.

It will be noted that the 20 triangles are naturally arranged
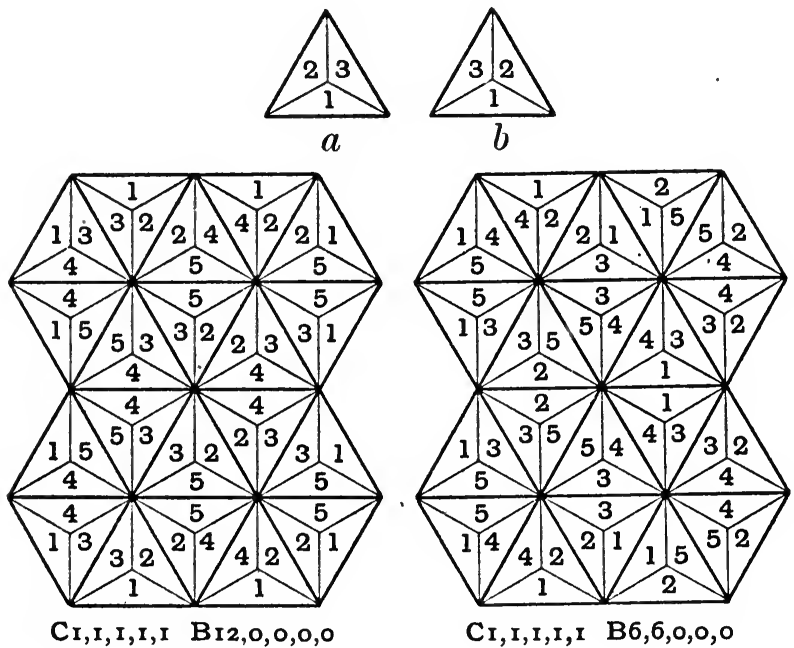

Fig. 19.

M. P. 
in pairs, because to every triangle (as fig. I9a) there can be associated the complementary (as fig. 19b).

The latter, read counterclockwise, is the same as the former read clockwise. This circumstance supplies a clue to the setting up of some of the boundary types. It is left to the reader to take advantage of this hint.

Some examples are given in fig. I9.

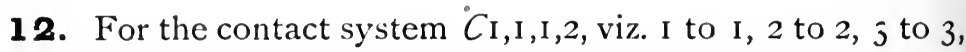
4 to 5 , the colours I, 2, 3 must each occur an even number of times upon the boundary and the colours 4,5 the same number of times.

The boundary types are 23 in number:

\begin{tabular}{rrr:rrr} 
Colours & I & 2 & 3 & 4 & 5 \\
\cline { 2 - 6 } & I 2 & 0 & 0 & 0 & 0 \\
IO & 2 & 0 & 0 & 0 \\
8 & 4 & 0 & 0 & 0 \\
8 & 2 & 2 & 0 & 0 \\
6 & 6 & 0 & 0 & 0 \\
6 & 4 & 2 & 0 & 0 \\
4 & 4 & 4 & 0 & 0 \\
IO & 0 & 0 & 1 & 1
\end{tabular}

\begin{tabular}{lllll} 
I & 2 & 3 & 4 & 5 \\
\hline 8 & 2 & 0 & I & I \\
6 & 4 & 0 & 1 & 1 \\
6 & 2 & 2 & 1 & 1 \\
4 & 4 & 2 & 1 & 1 \\
8 & 0 & 0 & 2 & 2 \\
6 & 2 & 0 & 2 & 2 \\
4 & 4 & 0 & 2 & 2 \\
4 & 2 & 2 & 2 & 2
\end{tabular}

\begin{tabular}{llll|ll} 
I & 2 & 3 & 4 & 5 \\
\hline 6 & 0 & 0 & 3 & 3 \\
4 & 2 & 0 & 3 & 3 \\
2 & 2 & 2 & 3 & 3 \\
4 & 0 & 0 & 4 & 4 \\
2 & 2 & 0 & 4 & 4 \\
2 & 0 & 0 & 5 & 5 \\
0 & 0 & 0 & 6 & 6
\end{tabular}

with varieties of every type except the first.

Examples are given in fig. 20.

$\mathrm{C}_{1, \mathrm{I}, \mathrm{I}, 2}$ Bo, 0,0,6,6

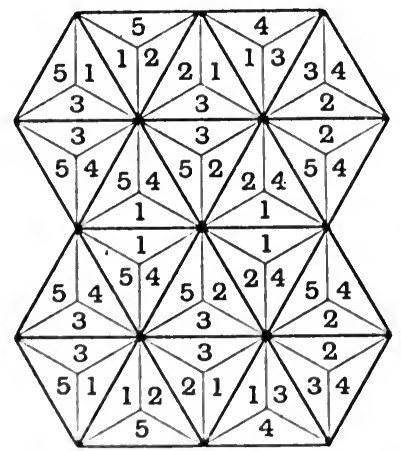

$\mathrm{C}_{1,1,1,2} \mathrm{BI}_{12,0,0,0,0}$

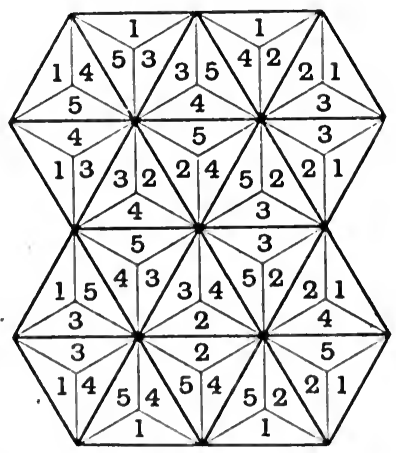

Fig. 20 . 


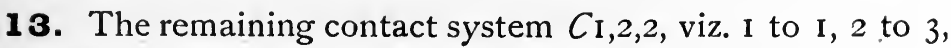
4 to 5 , yields the sixteen types:

\begin{tabular}{|c|c|c|c|c|c|c|c|c|c|c|c|c|c|c|}
\hline Colours & I & 2 & 3 & 4 & 5 & I & 2 & 3 & 4 & 5 & I & 2 & 3 & 4 \\
\hline & I2 & $\overline{0}$ & 0 & $\overline{\mathrm{O}}$ & $\overline{\mathrm{O}}$ & $\overline{4}$ & $\overline{4}$ & 4 & $\overline{0}$ & $\overline{0}$ & $\overline{2}$ & 3 & $\overline{3}$ & 2 \\
\hline & 10 & I & I & 0 & o & 4 & 3 & 3 & I & I & 0 & 6 & 6 & 0 \\
\hline & 8 & 2 & 2 & 0 & 0 & 4 & 2 & 2 & 2 & 2 & 0 & 5 & 5 & I \\
\hline & 8 & I & I & I & I & 2 & 5 & 5 & o & $\mathrm{O}$ & 0 & 4 & 4 & 2 \\
\hline & 6 & 3 & 3 & 0 & o & 2 & 4 & 4 & I & $I$ & o & 3 & 3 & 3 \\
\hline
\end{tabular}

with varieties of each type except the first.

Examples, which include two varieties of $B \circ, 3,3,3,3$, are given in fig. $2 \mathrm{I}$.

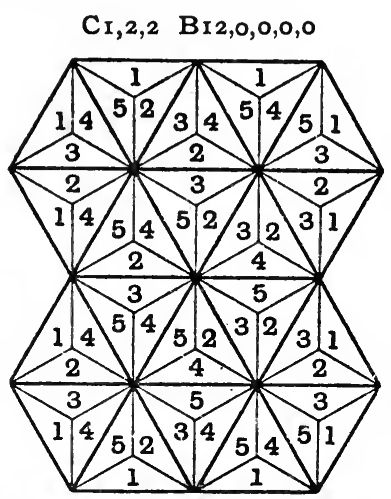

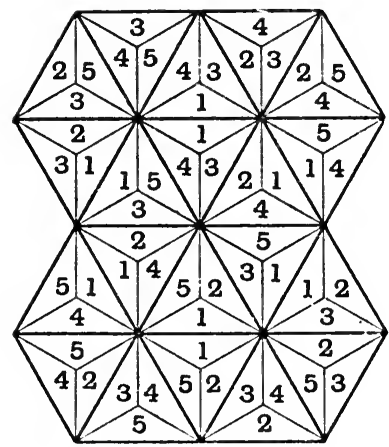

$\mathrm{Cr}_{1,2,2} \mathrm{Bo}, 3,3,3,3$

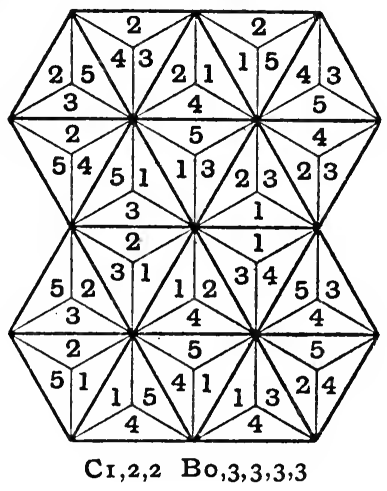

Fig. 2I.

This set is prolific of types, the three systems of contact yielding altogether 49 . 
Experiments with these will be found by many to be very interesting.

Eum odi sapientem qui sibi non sapit:

hee is an ill cooke that cannot licke his own fingers.

Withals' Dict., Ed. 1634.

14. We can divide the complete set into two portions:

(i) those which involve a particular colour, twelve in number ;

(ii) the remainder, eight in number, which involve only four colours I, 2, 3, 4 .

The first set of 12 is symmetrical in the four colours $1,2,3,4$.

There are 36 compartments which occur $6,6,6,6,12$ times for the five colours $\mathrm{I}, 2,3,4,5$ respectively.

The members of the set may be assembled in either of the forms of fig. 22, each of which has Io boundary compartments.
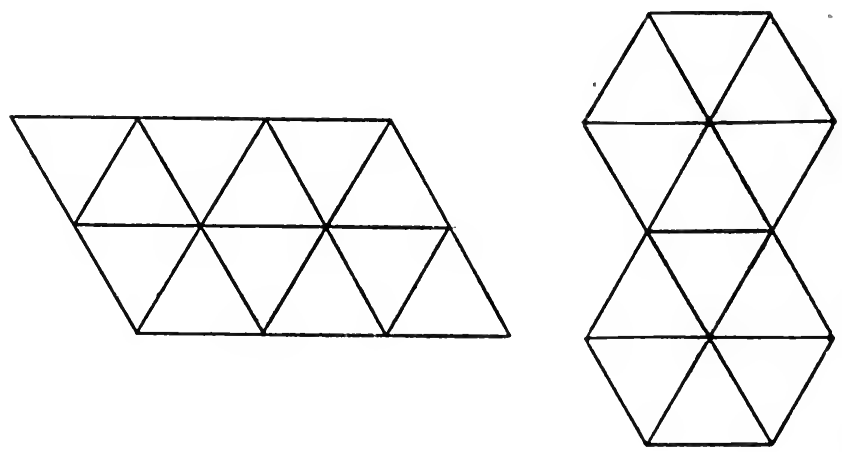

Fig. 22.

For the contact system $C_{\mathrm{I}, \mathrm{I}, \mathrm{I}, \mathrm{I}, \mathrm{I}}$ each colour must appear an even number of times upon the boundary, so that the types are fifteen in number:

Colours

\begin{tabular}{c|c|c|c|c} 
I & $\mathbf{2}$ & $\mathbf{3}$ & $\mathbf{4}$ & $\mathbf{5}$ \\
\hline $\mathrm{O}$ & $\mathrm{O}$ & $\mathrm{O}$ & $\mathrm{O}$ & $\mathrm{IO}$ \\
2 & $\mathrm{O}$ & $\mathrm{O}$ & $\mathrm{O}$ & 8 \\
4 & $\mathrm{O}$ & $\mathrm{O}$ & $\mathrm{O}$ & 6 \\
2 & 2 & $\mathrm{O}$ & $\mathrm{O}$ & 6 \\
$\mathrm{C}$ & $\mathrm{O}$ & $\mathrm{O}$ & $\mathrm{O}$ & 4
\end{tabular}

\begin{tabular}{ll|l|l|l} 
I & $\mathbf{2}$ & 3 & 4 & 5 \\
\hline 4 & 2 & $\mathrm{O}$ & $\mathrm{O}$ & $\mathbf{4}$ \\
2 & 2 & 2 & $\mathrm{O}$ & 4 \\
6 & 2 & $\mathrm{O}$ & $\mathrm{O}$ & 2 \\
4 & 4 & $\mathrm{O}$ & $\mathrm{O}$ & 2 \\
4 & 2 & 2 & $\mathrm{O}$ & 2
\end{tabular}

\begin{tabular}{l|l|l|l|l} 
I & 2 & 3 & 4 & 5 \\
\hline 2 & 2 & 2 & 2 & 2 \\
6 & 4 & 0 & 0 & 0 \\
6 & 2 & 2 & 0 & 0 \\
4 & 4 & 2 & 0 & 0 \\
4 & 2 & 2 & 2 & 0
\end{tabular}

Examples are given in fig. 23 . 
Certain of these types exist for only one of the two boundary shapes, as it is seen at once that the first of the types cannot exist for the parallelogram, while it does exist for the double hexagon.

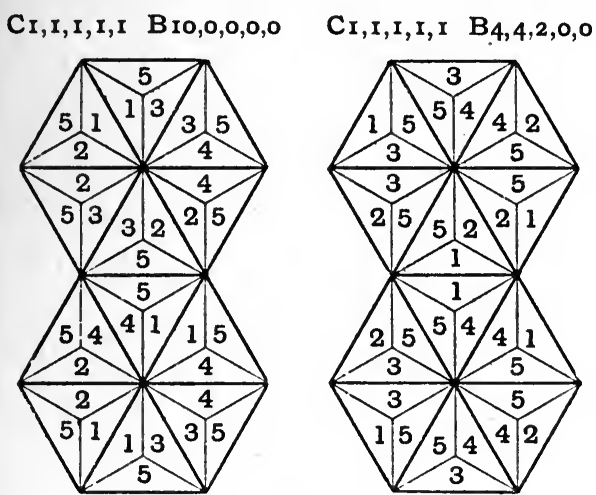

Fig. 23.

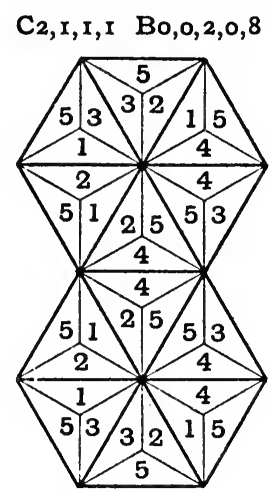

Fig. 24 .

15. For the contact system $C_{2, I, I, I}$ we find that the colours I, 2 must occur equally often and the colours $3,4,5$ each an even number of times. The types are 30 in number:

\begin{tabular}{|c|c|c|c|c|c|c|c|c|c|c|c|c|c|c|}
\hline Colours & I 2 & 3 & 4 & 5 & & I 2 & 3 & 4 & 5 & & 23 & 3 & & 5 \\
\hline & & 0 & 0 & 0 & 2 & 22 & 4 & 0 & 2 & & \begin{tabular}{l|l} 
I & 4
\end{tabular} & 4 & 4 & \\
\hline & 44 & 2 & 0 & 0 & 2 & 22 & 2 & 2 & 2 & & & o & o & 10 \\
\hline & 44 & 0 & 0 & 2 & 2 & 22 & 2 & 0 & 4 & & 0 & 2 & 0 & 8 \\
\hline & 33 & 4 & 0 & 0 & I & I I & o & 0 & 8 & & o & 4 & 0 & 6 \\
\hline & 33 & 0 & 0 & 4 & I & I I & 2 & 0 & 6 & & 0 & 2 & 2 & \\
\hline & 33 & 2 & 2 & 0 & I & I I & 4 & 0 & 4 & & 0 & 6 & & \\
\hline & 33 & 0 & 2 & 2 & & I I & 2 & 2 & 4 & & O & 4 & & \\
\hline & 22 & 6 & 0 & 0 & I & I I & 6 & 0 & 2 & & 0 & 6 & 2 & \\
\hline & 22 & 0 & 0 & 6 & I & I I & 4 & 2 & 2 & 0 & 0 & 4 & 4 & \\
\hline & 22 & 4 & 2 & 0 & I & I I I & 6 & 2 & 0 & 0 & & & & \\
\hline
\end{tabular}

An example is given in fig. 24 .

Some of these are probably non-existent for one or both forms of boundary.

We have further the contact systems

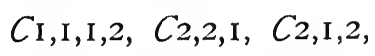

which may be left to the reader to examine.

How this geare will cotton I know not.

True Tragedie of Ric. III, I594. 
16. The second set of eight pieces puts up into the parallelogram of fig. 25 with eight boundary compartments.

The set is symmetrical in four colours. Each colour occurs on six compartments. The types for the different contact systems are

$$
C_{1,1,1, I}
$$

Colours
$\mathrm{C}_{2, \mathrm{I}, \mathrm{I}}$

\begin{tabular}{ll|l|l} 
I & 2 & 3 & 4 \\
\hline 4 & 4 & 0 & O \\
3 & 3 & 2 & 0 \\
2 & 2 & 4 & 0 \\
2 & 2 & 2 & 2 \\
I & I & 6 & 0 \\
I & I & 4 & 2 \\
O & O & 6 & 2 \\
O & 0 & 4 & 4
\end{tabular}

$\mathrm{C}_{2,2}$ \begin{tabular}{ll|ll}
1 & 2 & 3 & 4 \\
\hline 4 & 4 & 0 & 0
\end{tabular} $\begin{array}{llll}3 & 3 & \text { I } & \text { I }\end{array}$ \begin{tabular}{ll|ll}
2 & 2 & 0 & 0
\end{tabular}

Examples are given in fig. 25.

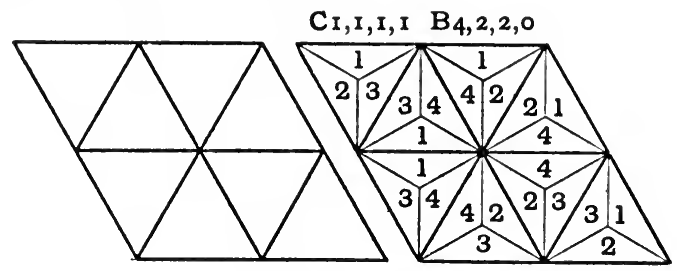

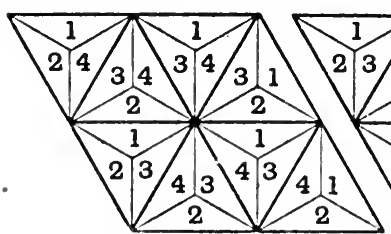

$\mathrm{C}_{2,2} \mathrm{~B}_{4,4,0,0}$

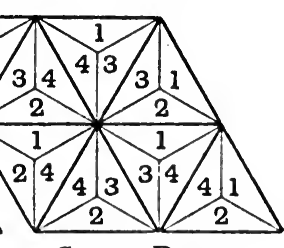

$\mathrm{C}_{2,1, \mathrm{I}} \mathrm{B}_{4,4}, \mathrm{O}$,

Fig. 25 . 


\section{SQUARE PASTIMES}

As a stream descending

From his fair heads to sea, becomes in trending

More puissant.

G. TOOKE's Belides.

17. Taking next the square, divide it into four equal and similar compartments by drawing the two diagonals and, as usual, regard each compartment as the location of a colour or number. Fixing upon three colours and allowing repetitions of colour in the compartments of the same square we obtain twenty-four different pieces as in fig. 26 which can obviously be arranged in a rectangle $6 \times 4$.

The 24 squares involve $4 \times 24$ or 96 compartments and, since each of the three colours is involved symmetrically, each must appear upon one-third of 96 or 32 compartments. 32 is an even

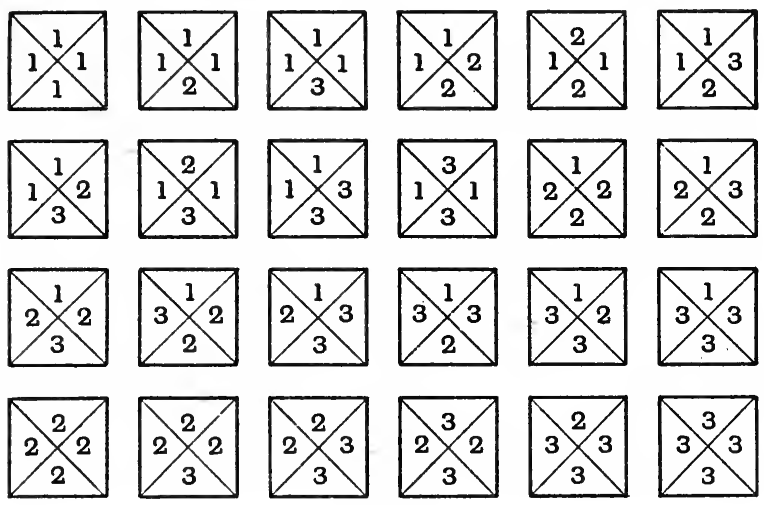

Fig. 26.

number and the rectangle has 20 boundary compartments so

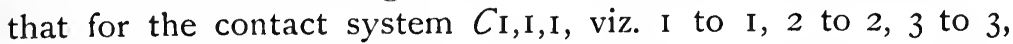
each colour must appear an even number of times upon the boundary. We have therefore the I4 types of boundary:

\begin{tabular}{cccc} 
Colours & I & 2 & 3 \\
\cline { 2 - 3 } 20 & 0 & 0 \\
I8 & 2 & 0 \\
16 & 4 & 0 \\
& 16 & 2 & 2 \\
& 14 & 6 & 0
\end{tabular}

\begin{tabular}{lrr} 
I & 2 & 3 \\
\hline I 4 & 4 & 2 \\
I2 & 8 & 0 \\
I2 & 6 & 2 \\
I2 & 4 & 4 \\
IO & 10 & 0
\end{tabular}

$\begin{array}{rrr}\text { I } & \mathbf{2} & \mathbf{3} \\ \text { IO } & 8 & 2 \\ \text { IO } & 6 & 4 \\ 8 & 8 & 4 \\ 8 & 6 & 6\end{array}$

with varieties of each type except the first. 
Most of these types have been actually arranged. Some of them are by no means easy but all are believed to be possible. Very little is known about the varieties of the different types.

The reader will not have much difficulty in setting up the first type $B 20,0,0$, with one colour everywhere upon the boundary, but he will find that thought and ingenuity are both required.

For as the precious stone diacletes, though it have many rare and excellent sovraignties in it, yet loseth them all, if it be put into a dead man's mouth.

Braith, Engl. Gent. p. 273.

An arrangement of type $B \mathrm{IO}, \mathrm{IO}, \mathrm{O}$ is given in fig. 27 .

CI, I, I Bro,10,0

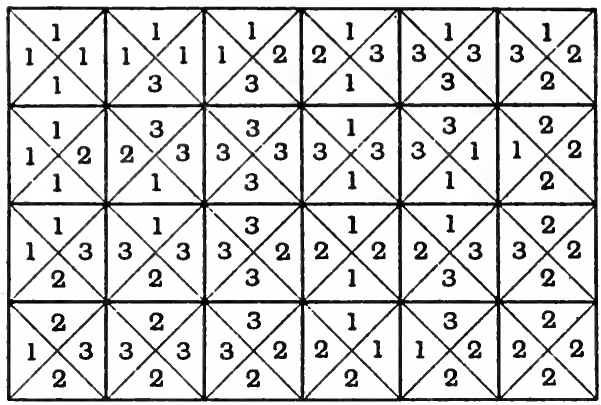

Fig. 27.

18. For the contact system $C_{\mathrm{I}, 2}$, viz. I to I, 2 to 3 , the colour I must occur an even number of times upon the boundary, whilst colours 2 and 3 must occur equally often.

We have the eleven types:

$\begin{array}{cccc}\text { Colours } & \text { I } & \mathbf{2} & \mathbf{3} \\ & 2 \mathrm{O} & \mathrm{O} & \mathrm{O} \\ \text { I } 8 & \text { I } & \text { I } \\ \text { I6 } & 2 & 2 \\ \text { I } 4 & 3 & 3 \\ \text { I } 2 & 4 & 4 \\ \text { IO } & 5 & 5\end{array}$

\begin{tabular}{rrr} 
I & 2 & 3 \\
\hline 8 & 6 & 6 \\
6 & 7 & 7 \\
4 & 8 & 8 \\
2 & 9 & 9 \\
0 & 10 & I0
\end{tabular}

with the usual varieties. Every type is believed to be a possible arrangement and two examples are given in fig. 28 .

Come on, sir frier, picke the locke,

This gere doth cotton hansome.

Troub. Reign of King John, p. I. 
$\mathrm{Cr}, 2 \mathrm{B20}, 0,0$

(1)

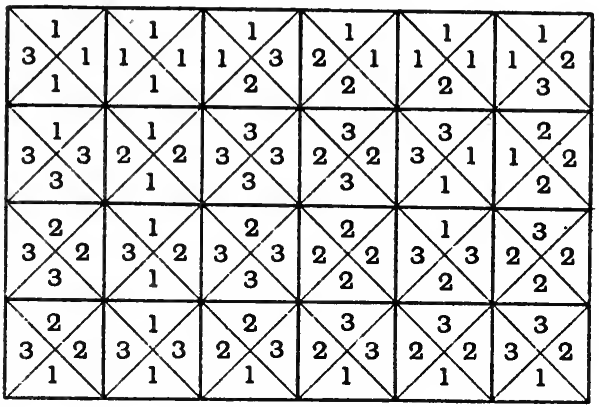

$\mathrm{Cr}, 2 \mathrm{Br}, 4,4$

Fig. 28.

Pleased with the same success, vast numbers press'd Around the shrine and made the same request.

The Temple of Fame.
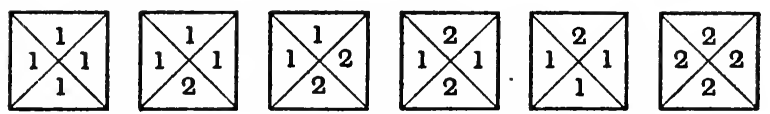

Fig. 29.

19. We may select from the complete set of 24 squares a lesser number upon definite principles.

If we are restricted to two colours we have only six squares as in fig. 29 which fit into a rectangle three squares by two.

The case is trivial from the present point of view, but since transformations of these diversions are effected in Part II of the book it is well not to leave it unexamined. 
Briefly the results are that for the contact system $C_{\mathrm{I}, \mathrm{I}}$ only one boundary type exists, viz. $B 6,4$, and for the contact system $C_{2}$ only one, viz. $B 5,5$.

Reflection, reason, still the ties improve, At once extend the interest and the love.

Essay on Man.

20. To make a more useful selection we may in the first place discard all squares which remain unaltered when the colours 2, 3 are interchanged. We thus discard the four squares of fig. 30 and are left with a set of 20 squares which can be assembled into a rectangle $5 \times 4$.
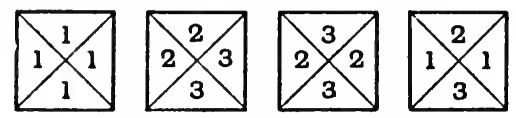

Fig. 30.

The set is not symmetrical in three colours, but only in the colours 2, 3 .

There are 18 boundary compartments and the colours I, 2, 3 occur in the set $26,27,27$ times respectively.

We see that on the boundary the colours must occur for the system $C_{\mathrm{I}, \mathrm{I}, \mathrm{I}}$

$$
\begin{aligned}
& \text { I an even number of times. } \\
& 2 \text { " uneven ", } \\
& 3 \text {, uneven }
\end{aligned}
$$

\begin{tabular}{|c|c|c|c|c|c|c|c|c|}
\hline Colours & I & 2 & 3 & I & 2 & 3 & I & 2 \\
\hline & 16 & I & I & 6 & 7 & 5 & 2 & I I \\
\hline & I 4 & 3 & I & 6 & 9 & 3 & 2 & 13 \\
\hline & I 2 & 3 & 3 & 6 & I I & I & 2 & 15 \\
\hline & I 2 & 5 & I & 4 & 7 & 7 & O & 9 \\
\hline & IO & 5 & 3 & 4 & 9 & 5 & O & I I \\
\hline & IO & 7 & I & 4 & I I & 3 & O & I 3 \\
\hline & 8 & 5 & 5 & 4 & I3 & I & O & 15 \\
\hline & 8 & 7 & 3 & 2 & 9 & 7 & O & I 7 \\
\hline & 8 & 9 & I & & & & & \\
\hline
\end{tabular}

There are 25 boundary types:

and the usual varieties. 
An example of the type $B 0,9,9$ is given in fig. $3 \mathrm{I}$.

\section{$\mathrm{Cl}_{\mathbf{I}, \mathbf{I}, \mathbf{I}}$ Bo,9,9}

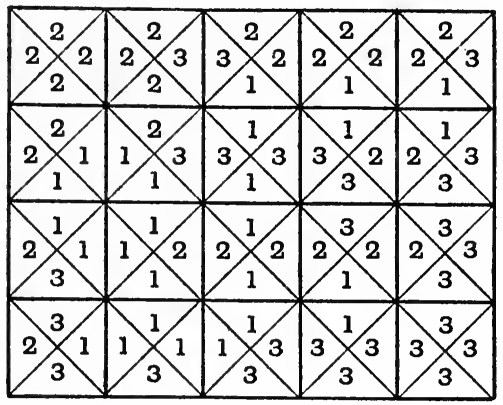

Fig. 3I.

21. For the contact system $C_{2}, \mathrm{I}$, viz. I to 2 and 3 to 3 , we find that colour 2 must occur once oftener than I upon the boundary and 3 an uneven number of times; this leads to nine types:

\begin{tabular}{cccc} 
Colours & $\mathbf{I}$ & $\mathbf{2}$ & $\mathbf{3}$ \\
\cline { 2 - 4 } & 8 & 9 & $\mathrm{I}$ \\
& 7 & 8 & 3 \\
& 6 & 7 & 5 \\
5 & 6 & 7 \\
4 & 5 & 9
\end{tabular}

\begin{tabular}{ccc} 
I & $\mathbf{2}$ & $\mathbf{3}$ \\
\hline 3 & 4 & I I \\
2 & 3 & I 3 \\
I & 2 & I 5 \\
O & I & I 7
\end{tabular}

These prove to be difficult to arrange, and some varieties of types appear not to be possible.

Hard or difficile be those thynges that be goodly or honest.

TAVERnER's Adagies, D. 5.

And when he had taryed there a long time for a convenable wind, at length it came about even as he desired.

HolinsHED'S Chron. I 577.

In view of Part II of this book it is desirable to obtain symmetrical boundaries for choice and one such example is given in fig. 32 for the type $B 2,3,13$.

22. For the contact system $C_{\mathrm{I}, 2}$, viz. I to $\mathrm{I}, 2$ to 3 , which exists by reason of the want of symmetry in three colours, we 
find colour I must occur an even number of times upon the boundary and colour 2 just as often as 3 .

$\mathrm{C}_{2, \mathrm{I}} \mathrm{B}_{2,3, \mathrm{I}}$

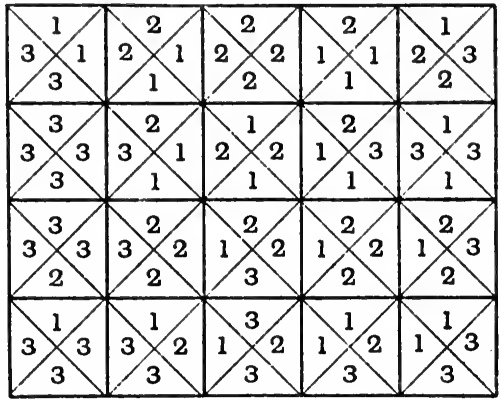

Fig. 32 .

There are ten types:

\begin{tabular}{llll} 
Colours & I & 2 & 3 \\
\cline { 2 - 4 } & I 8 & 0 & 0 \\
I6 & I & I \\
I4 & 2 & 2 \\
I2 & 3 & 3 \\
IO & 4 & 4
\end{tabular}

\begin{tabular}{lll} 
I & 2 & 3 \\
\hline 8 & 5 & 5 \\
6 & 6 & 6 \\
4 & 7 & 7 \\
2 & 8 & 8 \\
0 & 9 & 9
\end{tabular}

with varieties possibly of all except the first.

As an example a setting of $B \mathrm{I} 8,0,0$ is given in fig. 33 .

$\mathrm{C}_{1,2} \quad \mathrm{BI} 8,0,0$

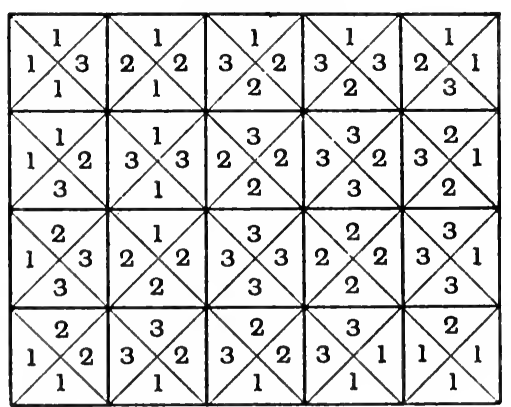

Fig. 33.

23. To reduce further the number of squares and, in the first place, with the object of obtaining a set of 16 we adopt as a discarding principle the rejection of all squares which involve a particular colour exactly twice and upon opposite compartments 
of the square. The reasons which prompt this rejection will appear in Part II, as it will be shewn therein that such pieces are inconvenient and even impracticable in the further developments. We thus reject the four squares of fig. $34 a$ and we are left with the 16 pieçes of fig. $34 b$.

The set is symmetrical in the two colours 2,3 but not in the three colours. They form up naturally into a square $4 \times 4$, which has 16 boundary compartments.

The colours I, 2, 3 occur upon $20,22,22$ compartments respectively.
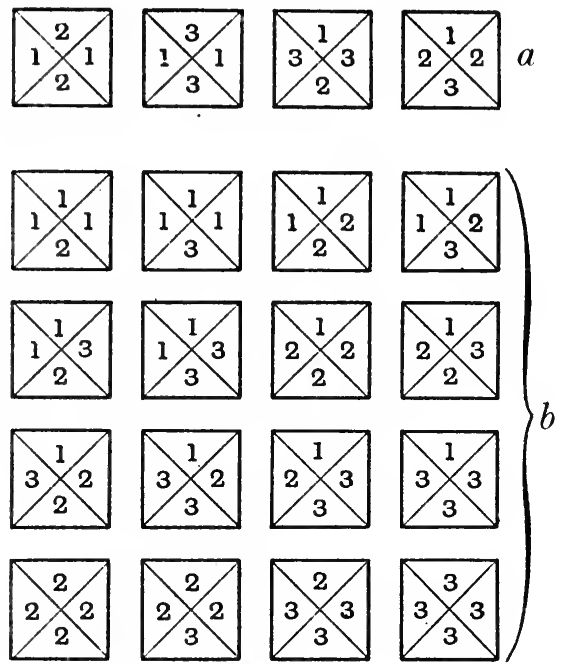

Fig. 34.

For the contact system $C_{\text {I,I, I }}$ each colour must appear an even number of times upon the boundary. We are led to 25 types of boundary:

Colours

\begin{tabular}{lll} 
I & 2 & 3 \\
\hline I6 & O & 0 \\
I4 & 2 & 0 \\
I2 & 4 & 0 \\
I2 & 2 & 2 \\
IO & 6 & 0 \\
IO & 4 & 2 \\
8 & 8 & 0 \\
8 & 6 & 2 \\
8 & 4 & 4
\end{tabular}

\begin{tabular}{rrr} 
I & 2 & 3 \\
\hline 6 & 10 & 0 \\
6 & 8 & 2 \\
6 & 6 & 4 \\
4 & 12 & 0 \\
4 & 10 & 2 \\
4 & 8 & 4 \\
4 & 6 & 6 \\
2 & 14 & 0 \\
2 & 12 & 2
\end{tabular}

with the usual varieties. 
It is not known how many of these exist.

He hath a person, and a smooth dispose

To be suspected.

Othello, I. iii.

As examples we have the three arrangements of fig. 35 in the first of which, $C_{\mathrm{I}, \mathrm{I}, \mathrm{I}} B_{\mathrm{I}} 6,0,0$, symmetry is apparent*.

\section{$\mathrm{C}_{\mathrm{I}, \mathrm{I}, \mathrm{I}} \mathrm{BI} 6,0,0$}

\begin{tabular}{|c|c|c|c|}
\hline $1 / 3$ & $1 / 3 / 2$ & $2 / 2 / 3$ & \\
\hline $1 / 3 / 3$ & $3 / 3 / 3$ & $3 / 2$ & \\
\hline $1 / 2 / 3$ & $3 / 3$ & $2 / 2$ & \\
\hline $\int_{1}^{2} / 2$ & $2 / 3$ & $32 / 1$ & \\
\hline
\end{tabular}

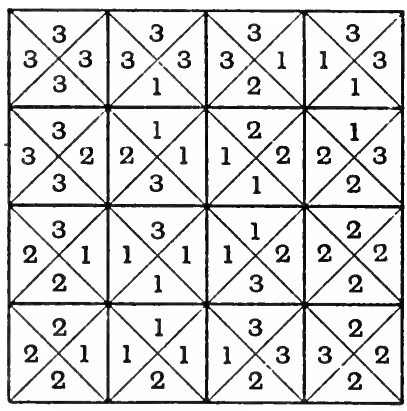

$\mathrm{CI}_{\mathrm{I}, \mathrm{I}, \mathrm{I}} \quad \mathrm{Bo}, 8,8$

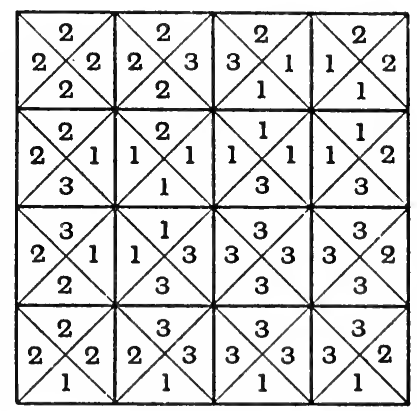

$\mathrm{C}_{\mathrm{I}, \mathrm{I}, \mathrm{I}} \quad \mathrm{B}_{4, \mathrm{I} 2, \mathrm{O}}$

Fig. 35 .

24. For the contact system $C_{2}, \mathrm{I}$, viz. I to 2,3 to 3 , the colour 2 must occur upon the boundary a number of times greater by two than the number of times I occurs, while colour 3 must occur an even number of times. The possible types are

and varieties.

\begin{tabular}{|c|c|c|c|c|c|c|}
\hline Colours & I & 2 & 3 & $\mathbf{I}$ & 2 & 3 \\
\hline & 7 & 9 & $\mathrm{O}$ & 3 & 5 & \\
\hline & 6 & 8 & 2 & 2 & 4 & IC \\
\hline & 5 & 7 & 4 & I & 3 & $\mathrm{I}_{2}$ \\
\hline & 4 & 6 & 6 & O & 2 & \\
\hline
\end{tabular}

* If any compartment be reflected through the centre of the whole square (the pointimagre) its colour if $\mathrm{I}$ is unchanged, but, if 2 or 3 , is changed to 3 or 2 respectively. 
Examples are given in fig. 36 .

$\mathrm{C}_{2, \mathrm{I}} \mathrm{Bo}, 2, \mathrm{I} 4$

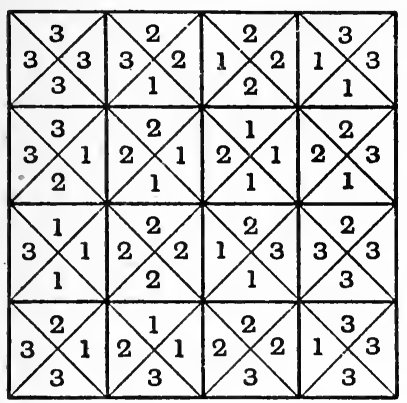

$\mathrm{C}_{2, \mathrm{I}} \mathrm{B}_{2,4}$, 10

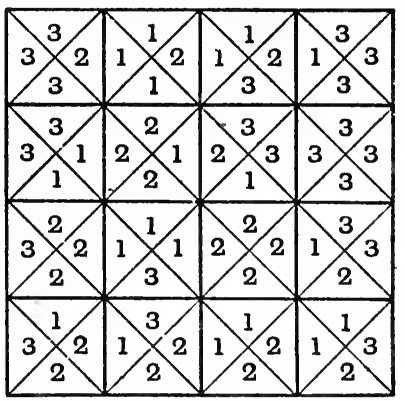

Fig. 36 .

25. For the contact system $C_{\mathrm{I}, 2}$, viz. I to I, 2 to 3 , we must have colour I an even number of times upon the boundary and colour 2 as often as 3 .

The types are nine in number:

\begin{tabular}{rrrrrrr} 
Colours & I & $\mathbf{2}$ & 3 & I & 2 & 3 \\
\cline { 2 - 6 } & I6 & O & 0 & 6 & 5 & 5 \\
& I4 & I & I & 4 & 6 & 6 \\
& I2 & 2 & 2 & 2 & 7 & 7 \\
IO & 3 & 3 & 0 & 8 & 8 \\
8 & 4 & 4 & & &
\end{tabular}

Examples are given in fig. 37.

$\mathrm{C}_{\mathrm{I}, 2} \mathrm{~B}_{\mathrm{I}} 6, \mathrm{O}, \mathrm{O}$

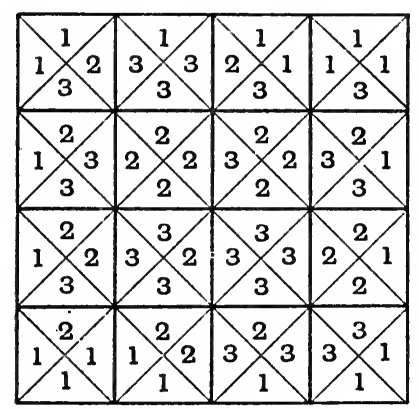

$\mathrm{CI}_{\mathrm{I}, 2} \mathrm{Bo}, 8,8$

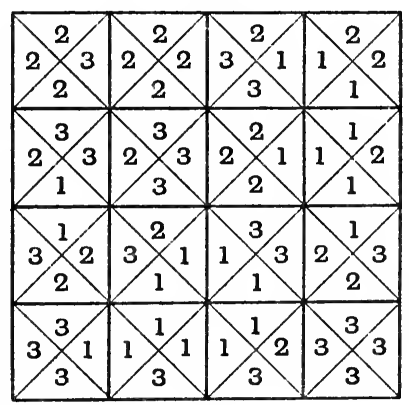

Fig. 37 . 
This set of pieces has been dealt with at some length because it possesses a large number of boundaries which are symmetrical in colour. Also because it lends itself particularly to the developments of Part II.

Yes, Sir, I study here the mathematics And distillation.

B. Jonson, Alch. iv. i.

The numbers $1,2,3, \ldots$, which have been used in connexion with the various sets, have been merely symbols for colours.

No use has been made of their arithmetical properties.

A single example will shew that it is possible to denote the colours by numbers and to call in the aid of arithmetic, in certain cases, to define the contact system that is before us.

In the last contact system dealt with above, viz.

$$
\begin{aligned}
& \text { I to I, } \\
& 2,3,
\end{aligned}
$$

substitute for the numbers $\mathrm{I}, 2,3$,

$$
\text { the numbers } \mathrm{I}, \mathrm{O}, 2 \text {, }
$$

so that the contacts in question are $\mathrm{I}, \mathrm{I}$ and $\mathrm{O}, 2$ as in fig. $38 a$ and we may define the conditions of contact to be that the sum of the numbers in adjacent compartments is 2 . Clearly we

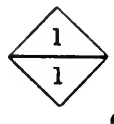

$a$

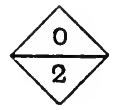

Fig. 38 .

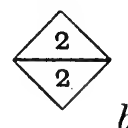

$b$

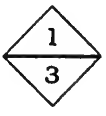

could increase each of the numbers $\mathrm{I}, \mathrm{O}, 2$ by the same number with the same result. Thence the simplest way of carrying out the idea is merely to interchange the numbers 1,2 so that the contacts are 2, 2 and 1,3 as in fig. $38 b$.

The sum of the numbers in adjacent compartments is then 4 .

In the case of five colours $1,2,3,4,5$ and the contact. system

$$
\begin{aligned}
& \text { I to I, } \\
& 2 \Rightarrow 3, \\
& 4 \Rightarrow 5,
\end{aligned}
$$

we similarly change the numbers

$$
\begin{aligned}
& \text { I, } 2,3,4,5 \\
& 3, I, 5,2,4
\end{aligned}
$$


respectively, and define the contact system to be such that the sum of the numbers in adjacent compartments is 6 .

26. There is an interesting set involving $I 5$ pieces which is derived from the set of 16 pieces, dealt with above, by omitting the pieces in fig. $39 a$ and adding the piece in fig. $39 b$.

This is a 15 -piece set which may be defined as involving the pieces of the original set of 24 :

(i) which have not more than three compartments of any piece of the same colour ;

(ii) which do not involve any piece which has exactly two compartments of the same colour and those opposite compartments.

The pieces will form up into a rectangle $5 \times 3$ as shewn by the figured rectangle in fig. $39 c$.

$$
\mathrm{CI}_{\mathrm{I}, \mathrm{I}, \mathrm{I}} \mathrm{BI} 6,0,0
$$
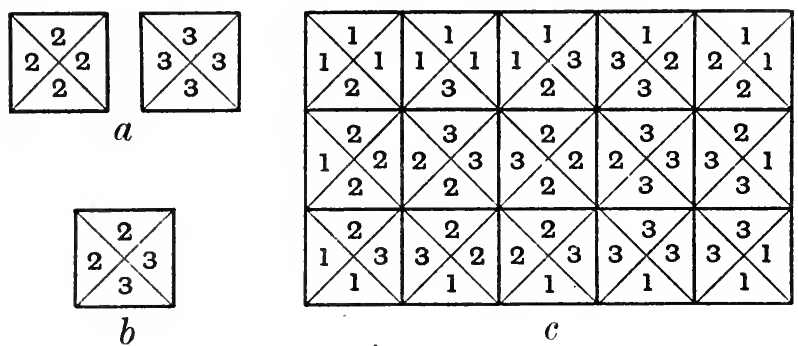

Fig. 39.

To analyse the set we note that it is symmetrical in the three colours and that the compartments coloured I, 2, 3 occur each 20 times.

There are 16 boundary compartments.

For the contact system $C_{\mathrm{I}, \mathrm{I}, \mathrm{I}}$ each colour must occur an even number of times upon the boundary.

The types are therefore

\begin{tabular}{cc|c|c} 
Colours & I & 2 & 3 \\
\cline { 2 - 3 } & I6 & O & 0 \\
I4 & 2 & 0 \\
I2 & 4 & 0 \\
I2 & 2 & 2 \\
IO & 6 & 0
\end{tabular}

\begin{tabular}{c|c|c} 
I & 2 & 3 \\
\hline IO & 4 & 2 \\
5 & 8 & 0 \\
8 & 6 & 2 \\
8 & 4 & 4 \\
6 & 6 & 4
\end{tabular}

ten in number.

M. P. 
27. For the contact system $C_{1,2}$, viz. I to 1,2 to 3 , the colour I must occur an even number of times upon the boundary and the colours 2, 3 equally often.

The types are

\begin{tabular}{|c|c|c|c|c|c|c|}
\hline \multirow[t]{6}{*}{ Colours } & I & 2 & 3 & I & 2 & 3 \\
\hline & 16 & 0 & o & 6 & 5 & ( \\
\hline & I 4 & I & I & 4 & 6 & 6 \\
\hline & I 2 & 2 & 2 & 2 & 7 & 7 \\
\hline & 10 & 3 & 3 & 0 & 8 & 8 \\
\hline & 8 & 4 & 4 & & & \\
\hline
\end{tabular}

nine in number.

All the types for both contact systems can probably be arranged and in many varieties.

Above, fig. $39 c$, is given an example of the type $B \mathrm{I} 6,0,0$ for

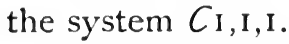

In fig. 40 is given one of the type $B_{14,1,1}$ for the system $C_{1,2}$.

$\mathrm{C}_{1,2} \mathrm{BI}_{4, \mathrm{I}, \mathrm{I}}$

\begin{tabular}{|c|c|c|c|c|}
\hline 1) 2 & $3 / 2$ & $3 / 3 / 3$ & $2 / 3$ & \\
\hline $2 / 3$ & $2 / 2 / 3$ & $2 / 3$ & $3 / 3$ & \\
\hline & $2 / 3$ & $2 / 2 / 2$ & & \\
\hline
\end{tabular}

Fig. 40.

28. A 9-piece set can be formed of the six pieces each of which involves three compartments of the same colour and also the three pieces each of which has two colours twice represented on either side of a diagonal. The set is given in fig. $4 \mathrm{I}$ assembling into a square $3 \times 3$.
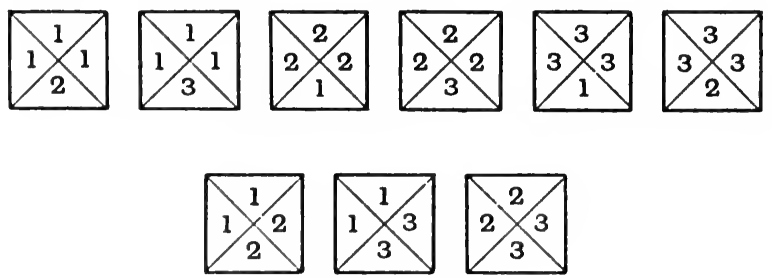

Fig. 4I. 
The set is symmetrical in three colours, each colour occurring in 12 compartments.

There are 12 boundary compartments.

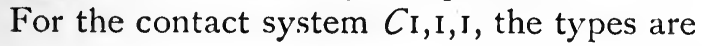

five in number.

\begin{tabular}{clll} 
Colours & $\mathbf{I}$ & $\mathbf{2}$ & $\mathbf{3}$ \\
\cline { 2 - 4 } & $\mathbf{8}$ & 4 & 0 \\
8 & 2 & 2 \\
6 & 6 & 0 \\
6 & 4 & 2 \\
4 & 4 & 4
\end{tabular}

29. For the contact system $C_{1,2}$, viz. I to $I, 2$ to 3 , the types are

five in number.

\begin{tabular}{llll} 
Colours & $\mathbf{I}$ & $\mathbf{2}$ & 3 \\
\cline { 2 - 4 } & 8 & 2 & 2 \\
& 6 & 3 & 3 \\
& 4 & 4 & 4 \\
& 2 & 5 & 5 \\
& 0 & 6 & 6
\end{tabular}

An arrangement of type $B 8,2,2$ for the system $C_{\mathrm{I}, 2}$ is given in fig. $42 a$, the boundary colours exhibiting symmetry about the diagonal $B D$.

$\mathrm{C}_{1,2} \mathrm{~B} 8,2,2$

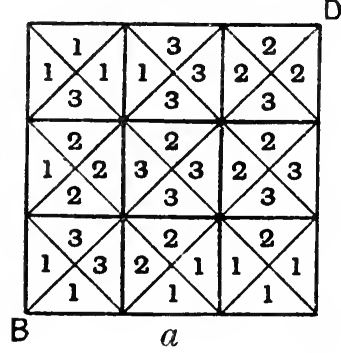

$\mathrm{C}_{1, \mathrm{I}, \mathrm{I}} \mathrm{B} 8,2,2$

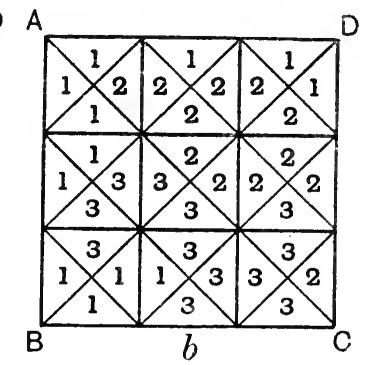

Fig. 42.

One of type $B 8,2,2$ for the system $C_{\mathrm{I}, \mathrm{I}, \mathrm{I}}$ is given in fig. $42 \mathrm{~b}$ in which considering the colour symmetry about the diagonal $A C$, it will be noticed that colour 2 appears along $D C$ in the same way as colour 3 appears along $B C$.

It is this kind of symmetry that leads, for this contact system, to the greatest symmetry in the transformations of Part II. 
30. In order to design a set based upon the square and involving five colours we adopt as a principle that every square is to involve four of the colours; there are to be no repetitions of colour in the compartments of the same square.

Four different colours give rise to six squares because four different objects have just six permutations in circular procession. We can select four colours out of five in five different ways so that we can obtain $5 \times 6$ or 30 different squares involving the five colours. There is little doubt that this would be an interesting set, but in this book we have limited ourselves to sets containing not more than twenty-four pieces and we can reduce the set of 30 as required by importing the condition that every piece is to involve one specified colour, say the colour I We thus get the set of twenty-four given in fig. 43 for which the rectangle $6 \times 4$ is available.

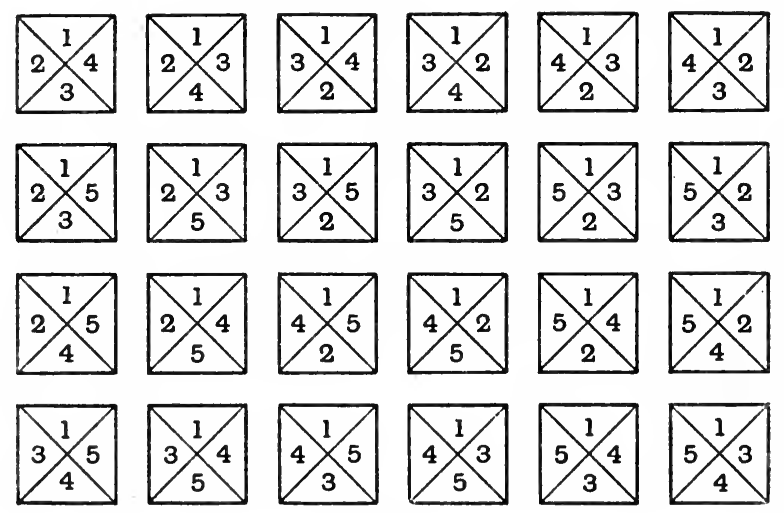

Fig. 43 .

The colours I, 2, 3, 4, 5 are involved upon $24, \mathrm{I} 8, \mathrm{I} 8, \mathrm{I} 8, \mathrm{I} \&$ compartments respectively. These are all even numbers and a: the number of boundary compartments is 20 , we have as condition that each colour must appear, in the contact system

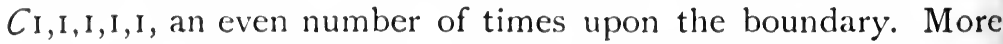
over each angular point of the rectangle must exhibit two different colours, so that a particular colour cannot appear upor the boundary more than 16 times. The number of boundary 
types is very large; it is not necessary to write them all down. They proceed for the colours

\begin{tabular}{lccccc}
\multirow{3}{*}{ from } & I & $\mathbf{2}$ & 3 & 4 & $\mathbf{5}$ \\
\cline { 2 - 5 } & I & 4 & 0 & 0 & 0 \\
to & $\vdots$ & $\vdots$ & $\vdots$ & $\vdots$ & $\vdots$ \\
& 0 & 6 & 6 & 4 & 4
\end{tabular}

Two examples are given in fig. 44 .

$\mathrm{C}_{\mathrm{I}, \mathrm{I}, \mathrm{I}, \mathrm{I}, \mathrm{I}} \mathrm{B}_{\mathrm{I} 4,0,0,4,2}$

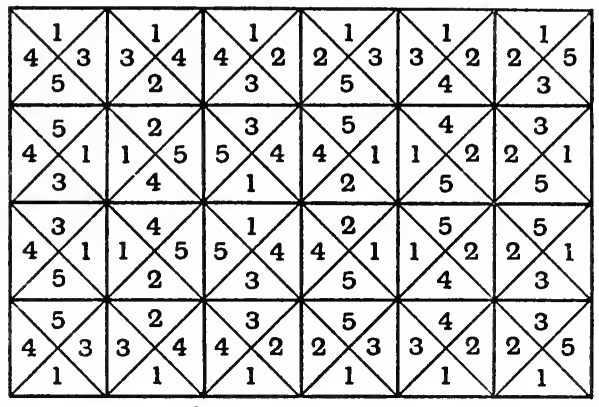

(1)

$\mathrm{C}_{\mathrm{I}, \mathrm{I}, \mathrm{I}, \mathrm{I}, \mathrm{I} \quad \mathrm{B} 6,6,2, \mathrm{O}, 6}$

Fig. 44.

Since there is only symmetry in four colours, four other contact systems are available:

\begin{tabular}{|c|c|c|c|}
\hline $\mathrm{C}_{2, \mathrm{I}, \mathrm{I}, \mathrm{I}}$ & $\mathrm{C}_{\mathrm{I}, 2, \mathrm{I}, \mathrm{I}}$ & $\mathrm{C}_{2,2, \mathrm{I}}$ & $\mathrm{C}_{1,2,2}$ \\
\hline I to 2 & I to I & I to 2 & I to I \\
\hline $3 » 3$ & 2,3 & $3 » 4$ & $2 " 3$ \\
\hline 4,4 & 4,4 & 5,5 & 4,5 \\
\hline $5 》 5$ & $5 " 5$ & & \\
\hline
\end{tabular}

The reader will know how to set forth the large numbers of boundary types that arise from these systems of contacts. 
31. Allusion has been made to varieties of the different types.

These should be chosen, for trial, so as to be symmetrical or semi-symmetrical about some axis passing through the centre of the figure. Thus in the case of the rectangle in fig. $45 a$ the chosen axis may be $A B$, or $C D$ or any other axis $E F$ passing through the centre of the figure.

If just two equally numerous colours are upon the boundary we may, for the axis $A B$, take just half of the compartments of each colour on each side of $A B$ and arrange them symmetrically.

Or we may make the compartments to the left of $A B$ any we please and then take a compartment, to the right, of the colour I when the corresponding compartment to the left has the colour 2.

Such arrangements for the axes $A B, E F$ might be as in fig. $45 b$ and $c$.
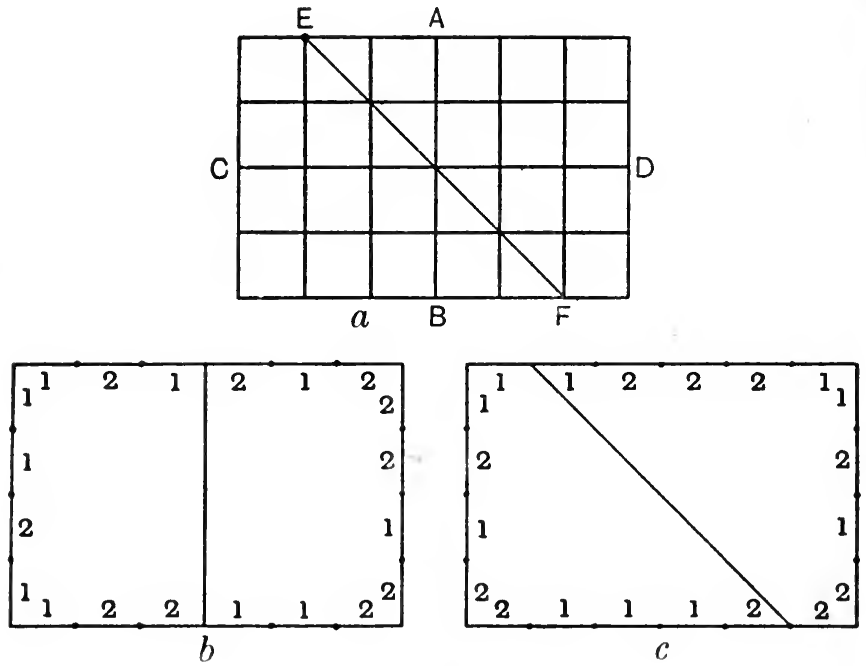

Fig. 45 .

These are extreme cases of what should be attempted.

The simplest case would be to place all the compartments of one of the colours on the same side of the axis chosen; and the simplest should always be chosen in the first place. When symmetrical boundaries are not practicable the interest is greatly diminished. It will be found that symmetrical boundaries lend themselves particularly to the transformations of Part II. 


\section{RIGHT-ANGLED TRIANGLE PASTIMES}

It cottons well, it cannot choose but beare A pretty napp.

Family of Love, D. 3 b.

32. Take a right-angled triangle, which is half of a square, as in fig. $46 a$.

Find its centre $O$ by taking $C O$ equal to two-thirds of the perpendicular drawn from $C$ upon $A B$. Then the three straight lines $C O, A O, B O$ divide the triangle into three compartments of equal areas.

If we have three colours and each triangle is to have three different colours in its compartments we obtain six different triangles which can be assembled, with the contact system $C_{\mathrm{I}, \mathrm{I}, \mathrm{I} \text {, }}$ in the form of fig. $46 b$ with any chosen colour monopolising the boundary.
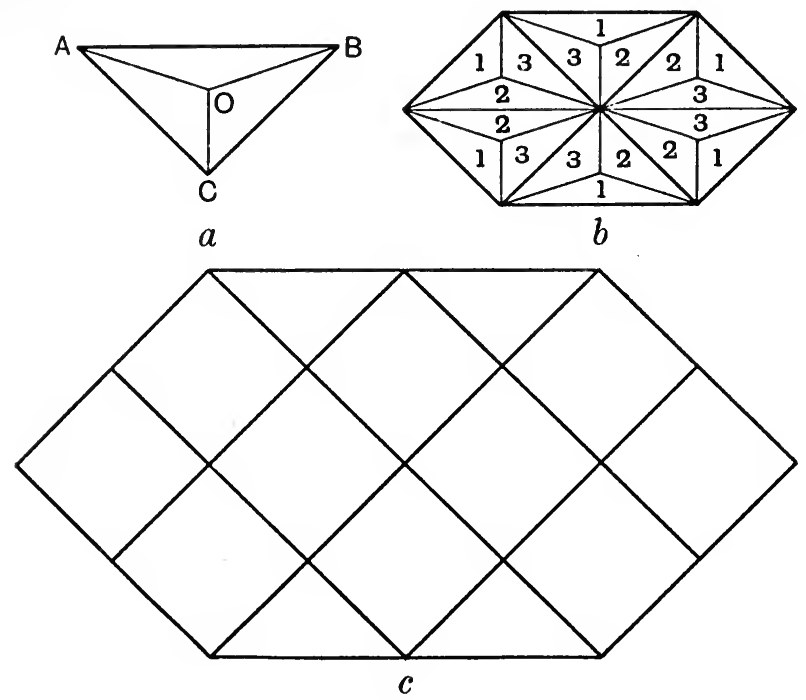

Fig. 46.

If we are given four colours we are led to 24 different triangles which can be arranged in the hexagonal form of fig. $46 c$, a figure consisting of four triangles and ten squares.

Two triangles may fit into any one of these squares with the long sides either vertical or horizontal. This is a new feature which adds interest to the study of the pieces. 
This is exhibited by two arrangements for the contact

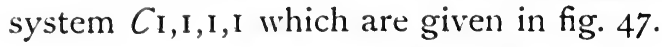
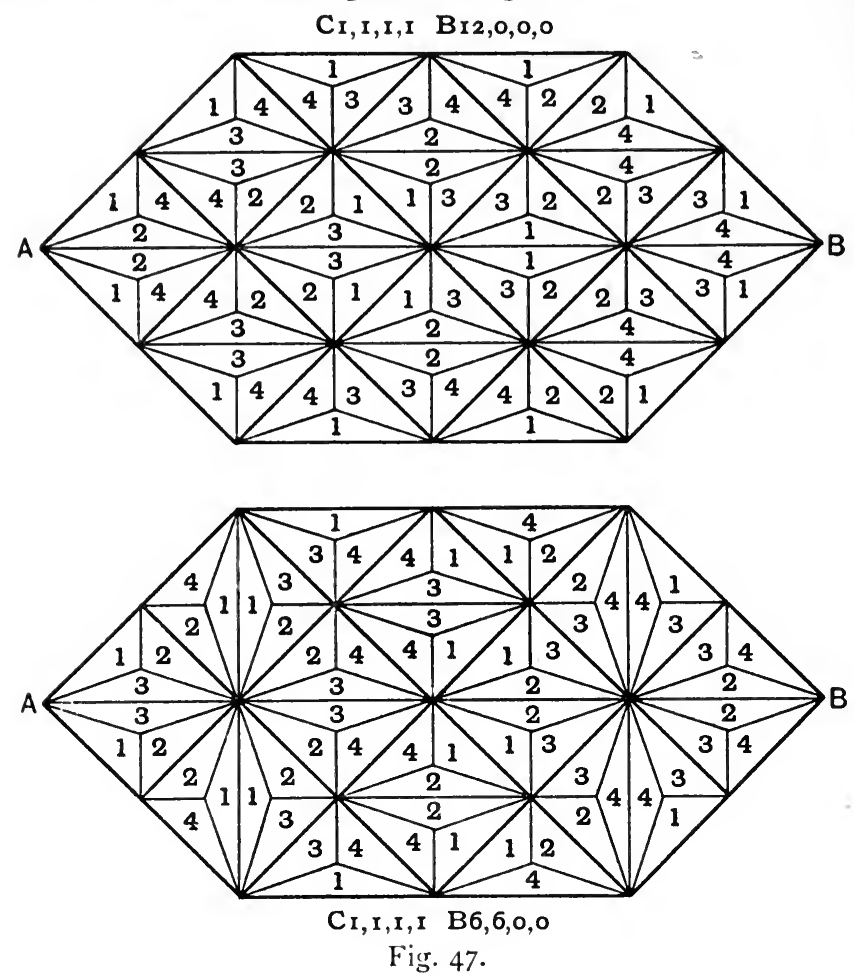

The first is symmetrical in colour about the axis $A B$.

The second differs from the first both in type of boundary and in internal structure.

In the first the triangles have two aspects, and in the second four aspects. In order to deal with the internal structure by type we may suppose the type to depend upon the numbers of triangles which have their long sides horizontal and vertical respectively. We might then describe the first arrangement by

and the second by

$$
\text { b'12,0,0,0 A24,0, }
$$

$$
B 6,6,0,0 \quad A 16,8 \text {. }
$$

Altogether there are 72 compartments, 24 long ( $L$ for long) and 48 short ( $S$ for short). Since there is symmetry in four colours, each colour appears upon $6 L$ and $12 S$ compartments. 
Upon the boundary there are $4 L$ and $8 S$. Hence for the

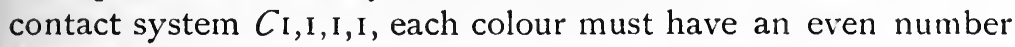
of $L$ and an even number of $S$ compartments upon the boundary.

So that as regards $L$ we have for

$$
\begin{array}{llllll}
\text { Colours } & \mathbf{I} & \mathbf{2} & \mathbf{3} & 4 & \\
\cline { 2 - 6 } & 4 & 0 & 0 & 0 & \\
2 & 2 & 0 & 0 & \text { two types ; }
\end{array}
$$

and as regards $S$ we have for

$$
\begin{array}{llllll}
\text { Colours } & \text { I } & \mathbf{2} & \mathbf{3} & 4 & \\
& 8 & 0 & 0 & 0 & \\
6 & 2 & 0 & 0 & \\
4 & 4 & 0 & 0 & \\
4 & 2 & 2 & 0 & \\
2 & 2 & 2 & 2 & \text { five types ; }
\end{array}
$$

with numerous varieties both in internal structure and in boundary.
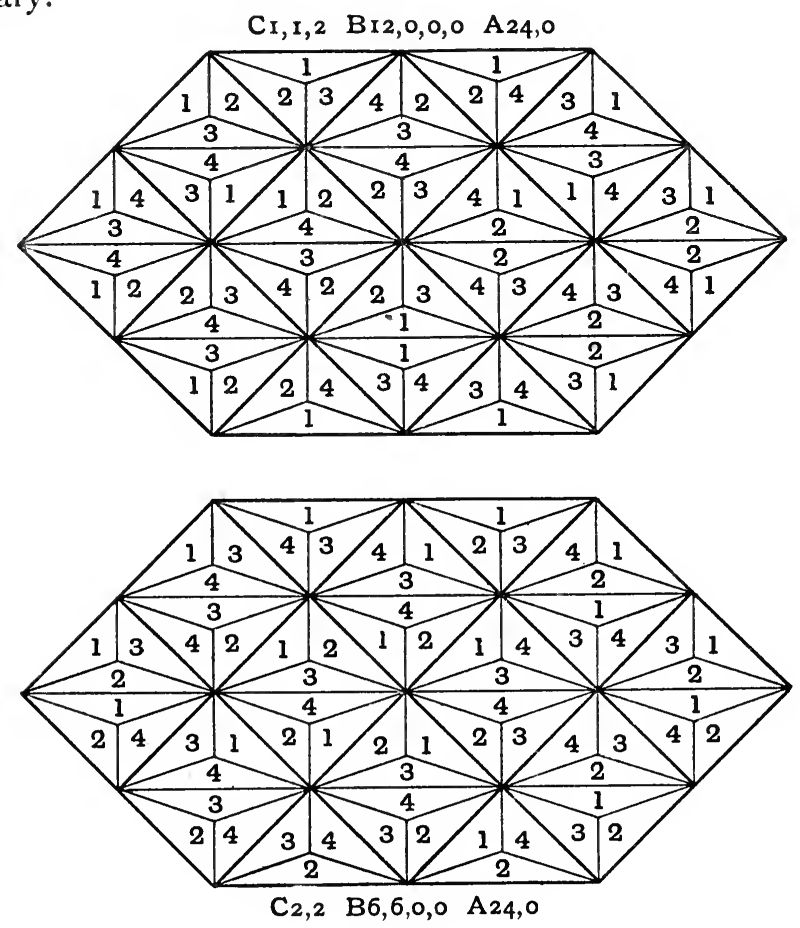

Fig. 48. 
We must presume that each of the $L$ types may occur with each of the $S$ so that there may be ten types in all.

Also of internal structure we may have the eleven types $A 240, A 22,2, A 20,4, A$ I 8,6, $A$ i6,8, $A$ I4,10, $A$ i 2, I 2,
$A_{\text {I0, I } 4, A 8,16, A 6,18, A 4,20,}$ and of every type of boundary except one and of every type of internal structure except one there may be varieties.

In regard to other contact systems, there is no reason why the $L$ compartments should have the same system as the $S$, because they do not clash at all.

33. Other systems available for both $L$ and $S$ are

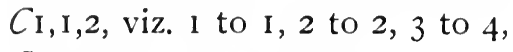

and

$$
\text { C2,2, viz. I to } 2,3 \text { to } 4 \text {. }
$$

Assemblages for the systems (for both $L$ and $S$ ) $C_{\mathrm{I}, \mathrm{I}, 2, C_{2,2}}$ are given in fig. 48 .

\section{THE CUBE P.ASTIME}

34. A cube has six faces, twelve edges and eight summits.

If we are allowed six different colours in order to colour the faces each with a different colour, we find that we can make 30 differently coloured cubes.

It is a well-known rule, applicable to any regular solid, that in order to ascertain the number of different cubes or other solids that can be made by colouring the faces with different colours it is merely necessary to divide the factorial of the number of faces by twice the number of edges. Thus in the case of the cube we have

$$
\frac{6 \times 5 \times 4 \times 3 \times 2 \times 1}{2 \times 12}=30 .
$$

So also in the case of the tetrahedron, composed of four equilateral triangles, which has four faces and six edges we have

$$
\begin{gathered}
4 \times 3 \times 2 \times 1 \\
2 \times 6
\end{gathered}=2,
$$

and so on for any regular solid.

IVe now construct these 30 cubes and, denoting the colours by numbers, we represent any such cube in a diagram as in 
fig. $49 a$, the cube being supposed resting upon a table and viewed from above.

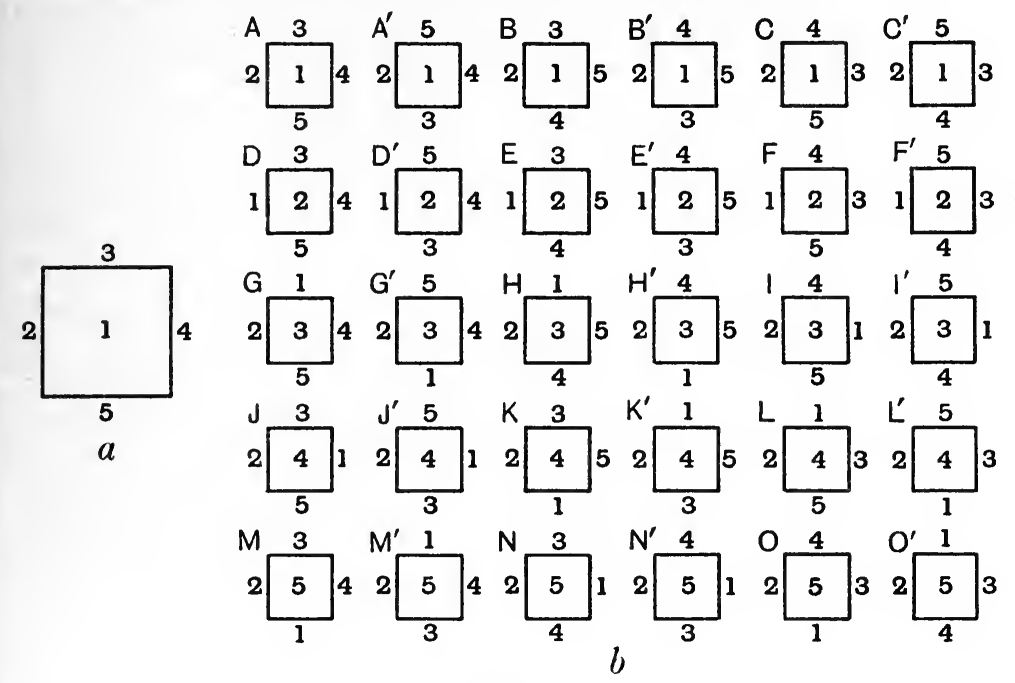

Fig. 49 .

Thus we are looking straight down upon the uppermost face coloured I and see the edges of the four vertical faces which are numbered 2, 3, 4, 5 respectively: the faces are read always in clockwise order.

The face numbered 6 is not visible, so that the number 6 does not appear in the diagram but is regarded as being present but obscured from view by the mass of the cube.

This being understood the 30 cubes are as given in fig. $49 b$.

They are conveniently denoted by 5 capital letters and by the same letters dashed, because they naturally arrange themselves into I 5 pairs of cubes.

For example the cubes denoted by $G, G^{\prime}$ have the same pairs of opposite faces; the faces coloured I, 2, 3 are opposite to those coloured $5,4,6$ respectively in both cubes. If the colours upon any pair of opposite faces of one of the cubes be interchanged the other cube is produced.

Looking vertically down upon the cubes the colours read clockwise on the one are identical with the colours read counterclockwise upon the other; the readings having reference of course to the vertical faces. 
The above cubes are called 'associated cubes.'

It is not obvious or even very easy to construct a Pastime from these 30 cubes. They can be assembled into a block having the dimensions $2 \times 3 \times 5$ and we can make selections from the whole number in many ways; for instance if we can select intelligently either 8 or 27 of these they can be assembled into large cubes. Moreover we have four different contact systems at our disposal which, following the practice of other

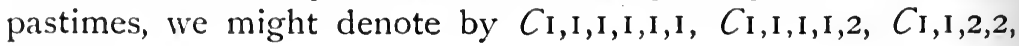
C2,2,2.

It is now some years since Colonel Julian R. Jocelyn communicated to the present writer the fact that he could select eight cubes

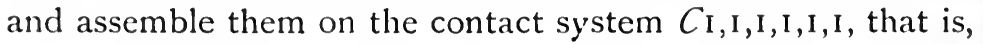

$$
\text { I to I, } 2 \text { to } 2,3 \text { to } 3,4 \text { to } 4,5 \text { to } 5,6 \text { to } 6 \text {, }
$$

so as to produce a cube of twice the linear dimensions which is a faithful copy in colours of any given member of the set of thirty cubes.

Suppose that it is desired to thus produce the cube denoted by $A$.

The two cubes $A, A^{\prime}$ have the opposite pairs I $-6,2-4,3-5$.

Reject from the complete set all the cubes which have any pair of these opposites and it will be found that we are left with the following 16 , viz:

$$
\begin{aligned}
& E, F, H, I, K, L, N, O, \\
& E^{\prime}, F^{\prime}, H^{\prime}, I^{\prime}, K^{\prime}, L^{\prime}, N^{\prime}, O^{\prime} .
\end{aligned}
$$

These may be further divided into two sets, each of eight cubes, viz. :

first set

second set

$$
\begin{aligned}
& K, L, F^{\prime}, E^{\prime}, H^{\prime}, O^{\prime}, I, N, \\
& K^{\prime}, L^{\prime}, F, E, H, O, I^{\prime}, N^{\prime},
\end{aligned}
$$

which are connected with the cubes $A, A^{\prime}$ respectively.

The first set may be assembled, with the contact system

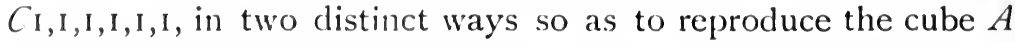
on twice the linear scale. The first solution is shewn in fig. $50 a$, the second solution in fig. 506 , the result, in each case, being as in fig. $50 \mathrm{c}$.

The two solutions are connected in an interesting manner. A straight line which passes from any summit of a cube through 
the centre of the cube to another summit is a diagonal of the cube. There are four diagonals. Each of the eight cubes which compose the large cube is diagonally opposite to another cube.

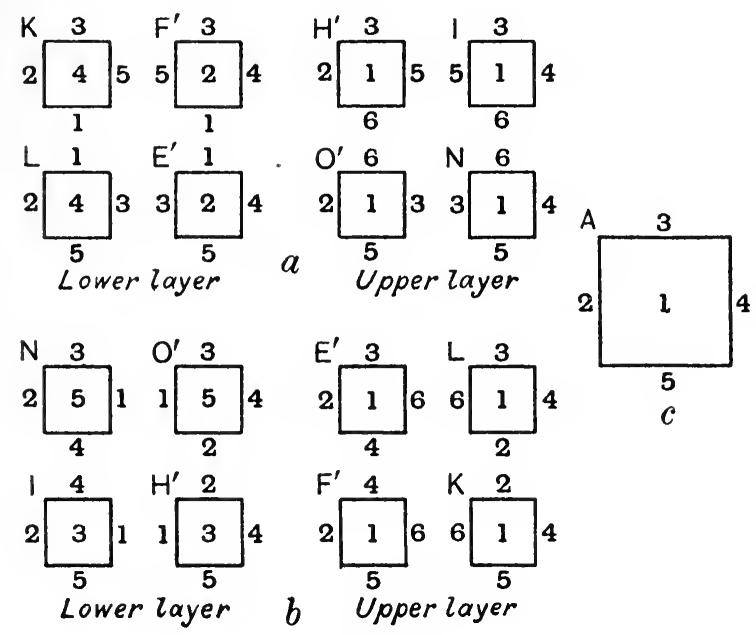

Fig. 50 .

We see that in each solution there is $n o$ change in the diagonally opposite pairs. In the first solution, for example, we have the pair $F^{\prime} O^{\prime}$ corresponding to the pair $O^{\prime} F^{\prime}$ in the second. All that has happened is that the components of the pair have become interchanged.

The eight cubes in either case involve 48 faces and of these exactly half, viz. 24, are boundary faces. The remaining 24 are inside faces. It is a remarkable circumstance that the 24 boundary faces in the first solution are inside faces in the second and vice versa.

The geometry of the solutions can be further studied by taking advantage of the fact that the six centres of the six faces of a cube are the summits of a regular octahedron*. The geometrical reader may be interested in following up this point.

In order to make an interesting property of the set of 30 cubes clear, it is convenient to speak of the large cube $A$ as containing each of the component cubes $K, L, F^{\prime}, E^{\prime}, H^{\prime}, O^{\prime}, I, N$.

* Proceedings of the London Mathematical Society, Vol. 24 , p. 145, 1893 . 
In this language the complete results may be set forth as follows :

\begin{tabular}{|c|c|c|c|c|c|}
\hline \multicolumn{3}{|c|}{$A$ contains $K L F^{\prime} E^{\prime} H^{\prime} O^{\prime} I Y$} & \multicolumn{3}{|c|}{$A^{\prime}$ contains $F E K^{\prime} L^{\prime} I^{\prime} N^{\prime} \cdot H O$} \\
\hline$B$ & , & $M O^{\prime} F D^{\prime} G^{\prime} L I^{\prime} J$ & $B^{\prime}$ & , & $F^{\prime} D M^{\prime} O I J^{\prime} G L^{\prime}$ \\
\hline C & $"$ & $H^{\prime} G D^{\prime} E K M^{\prime} J N^{\prime}$ & $C^{\prime}$ & " & $D E^{\prime} H G^{\prime} J^{\prime} N K^{\prime} M$ \\
\hline$D$ & , & $L K C^{\prime} B^{\prime} G M I^{\prime} N^{\prime}$ & $D^{\prime}$ & & $C B L^{\prime} K^{\prime} I N G^{\prime} M^{\prime}$ \\
\hline$E$ & ", & $O^{\prime} M C A^{\prime} H K I J^{\prime}$ & $E^{\prime}$ & & $C^{\prime} A O M^{\prime} I^{\prime} J H^{\prime} K^{\prime}$ \\
\hline$F$ & $"$ & $G H^{\prime} A^{\prime} B L O \Gamma^{\prime} N$ & $F^{\prime}$ & & $A B^{\prime} G^{\prime} H J N^{\prime} L^{\prime} O^{\prime}$ \\
\hline$G$ & , & $C B^{\prime} O^{\prime} N F K^{\prime} D J$ & $G^{\prime}$ & & $O N^{\prime} C^{\prime} B D^{\prime} J^{\prime} F^{\prime} K$ \\
\hline$H$ & & $C^{\prime} A^{\prime} L J F^{\prime} M^{\prime} E N$ & $H^{\prime}$ & & $L^{\prime} J^{\prime} C A E^{\prime} N^{\prime} F M$ \\
\hline$I$ & & $B^{\prime} A E D^{\prime} K^{\prime} M L O$ & $I^{\prime}$ & & $E^{\prime} D B A^{\prime} L^{\prime} O^{\prime} K M^{\prime}$ \\
\hline J & , & $B C E^{\prime} F^{\prime} H O G M$ & $J^{\prime}$ & & $E F B^{\prime} C^{\prime} G^{\prime} M^{\prime} H^{\prime} O$ \\
\hline$K$ & & $G^{\prime} I^{\prime} A C E N D O$ & $K^{\prime}$ & & $A^{\prime} C^{\prime} G I D^{\prime} O^{\prime} E^{\prime} N^{\prime}$ \\
\hline$L$ & & $N^{\prime} M^{\prime} B A I F H D$ & $L^{\prime}$ & & $B^{\prime} A^{\prime} N M H^{\prime} D^{\prime} I^{\prime} F^{\prime}$ \\
\hline$M$ & & $J L^{\prime} D E I C^{\prime} H^{\prime} B$ & $M^{\prime}$ & & $D^{\prime} E^{\prime} J^{\prime} L H B^{\prime} I^{\prime}$ \\
\hline$N$ & & $L^{\prime} H K$ & $N^{\prime}$ & & $D F^{\prime} A^{\prime} C H^{\prime} K^{\prime} G^{\prime} L$ \\
\hline$O$ & $"$ & $A^{\prime} B^{\prime} J K G^{\prime} E^{\prime} I F$ & $O^{\prime}$ & $"$ & $J^{\prime} K^{\prime} A B I^{\prime} F^{\prime} G$ \\
\hline
\end{tabular}

The property for observation is that if any cube $X$ contains the cube $Y$, then reciprocally the cube $Y$ contains the cube $X$.

For example $K$ being in the set of eight which compose $A$, it will be found on inspection of the results that $A$ is in the set which compose $K$.

The first result, which specifies the cubes contained by $A$, shews at once the eight cubes, each of which contains $A$.

The geometry of the octahedron referred to above reveals the reason for this reciprocity.

It is probable that much more remains to be discovered concerning the properties of the set of 30 cubes. The writer has no doubt that clever readers will find out other selections and contact systems which have escaped his own observation.

As a Pastime it is not difficult to assemble any given set of eight cubes if the cube to be assembled is known beforehand; but it is not nearly so easy if this knowledge be withheld from the solver. It is not however, in that case, a mere matter of chance; there is ample opportunity, as in all of these Pastimes, for the exercise of thought and cleverness.

35. The circumstance, that it is possible to select eight cubes from the complete set which, on a defined contact system, 
reproduce on a larger scale a particular cube selected from the complete set, has its parallel in the Triangle and Square Pastimes in the earlier pages of this book. Thus if we take the set of 20 equilateral triangles associated with five colours, not repeatable, we find that four triangles may be selected which will reproduce on twice the scale any chosen member of the set. Not only so, but nine triangles may be selected so as to reproduce it on three times the scale and sixteen triangles may be chosen so as to reproduce it on four times the scale, as in fig. 5 I where arrangement $a$ reproduces triangle $b$ on four times the scale.

Also in the case of the set of 24 right-angled triangles connected with four colours, not repeatable, we can select four triangles with the same result as in fig. 5 I $c$ and $d$. The same property is possessed by the squares associated with non-repeatable colours as shewn in fig. $5 \mathrm{I} e$ and $f$.

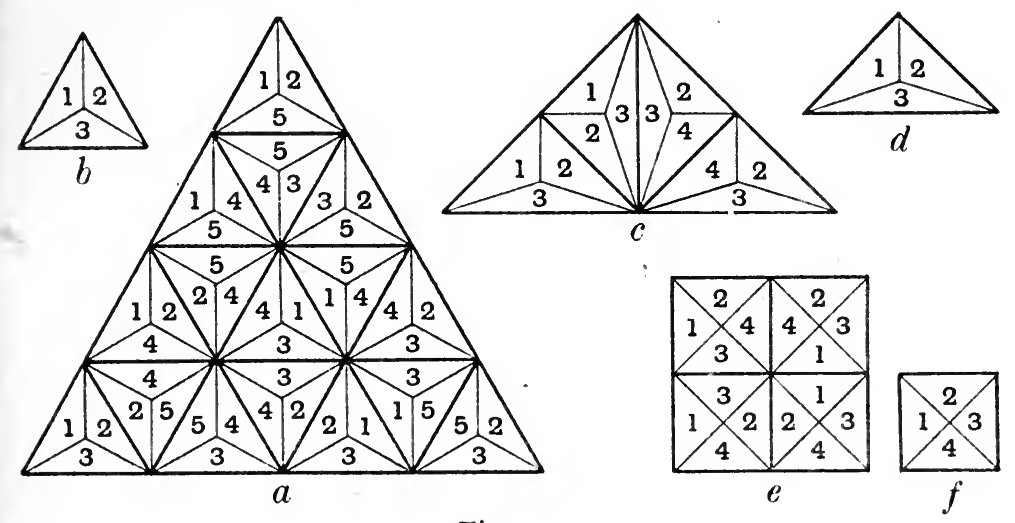

Fig. $5 \mathrm{I}$.

\section{PASTIMES BASED UPON THE REGULAR HEXAGON}

As our degrees are in order distant,

So the degrees of our strengths are discrepant.

HEYwOod's Spider and Flie, 1556.

36. The compartments of the hexagon in the first row of fig. 52 are numbered in circular order. We must define some law with regard to the numbers, or to the colours which they may denote, which leads to a convenient number of hexagons. If we have six different colours upon each hexagon we are led to a set of 120 because, placing a particular colour, say 6 , upper- 
most, we can permute the remaining colours in $5 \times 4 \times 3 \times 2 \times 1$ different ways.

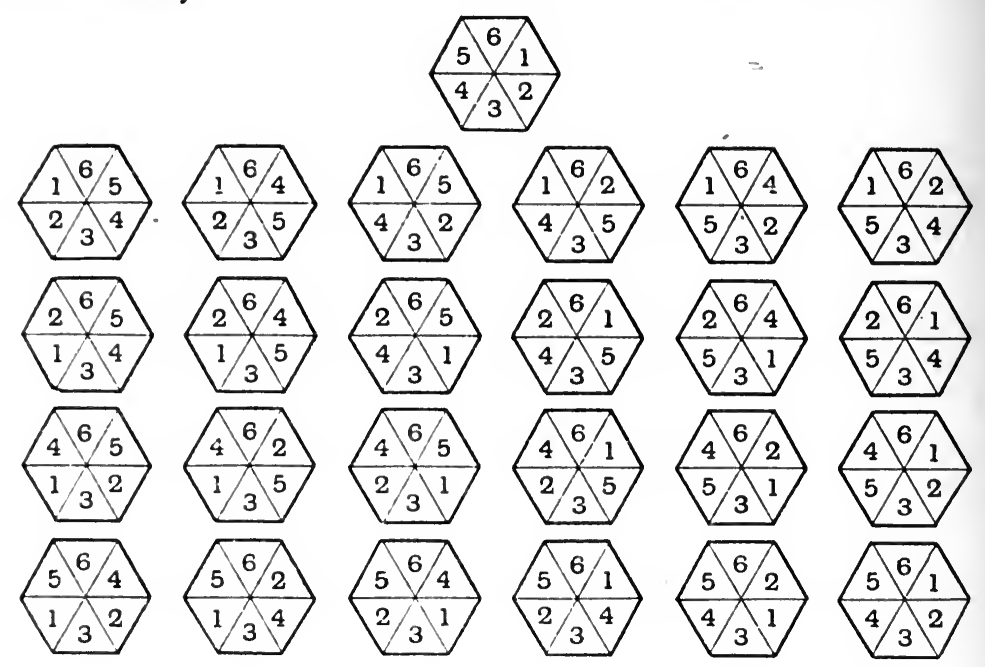

Fig. 52.

If however we not only place the colour 6 uppermost but also another colour, say 3, downmost, the remaining colours can only be placed in $4 \times 3 \times 2 \times$ I or 24 different permutations.

We shall then have the convenient set of 24 as in fig. 52 (last four rows).

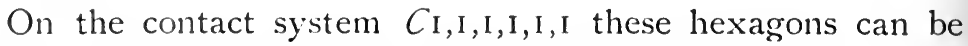
arranged in the shapes shewn on a smaller scale in fig. 53.

'The forms of assemblage marked I, II, III have 38,40 and 36 boundary compartments respectively.

The set of 24 hexagons involves the compartments coloured I, 2, 3, 4, 5, 6, each 24 times. It follows that for the contact

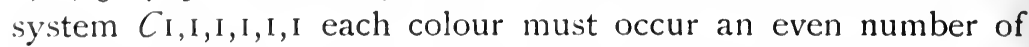
times upon the boundary in each of the three assemblages.

The two colours denoted by 3,6 are in an exceptional position because they are opposite upon each piece.

If we consider any piece in either of the three assemblages and join the centres of its 3, 6 compartments, this line produced both ways will always pass through 3 and 6 compartments (alternately) and through no other until the two opposite boundaries are reached. Further, any straight line joining the 
centres of 3 and 6 compartments of any other piece in the assemblage must be parallel to the foregoing line.

It follows at once that, since the boundary is made up of lines going in three different directions, one of these directions must involve the colours 3 and 6 exclusively.

We examine the boundaries of the assemblages I, III and discover that the colours 3,6 together must occupy either 16 or

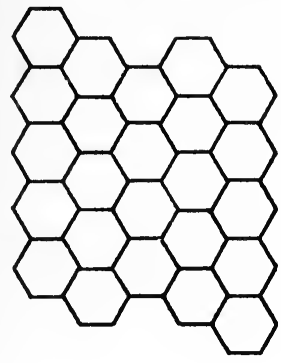

1

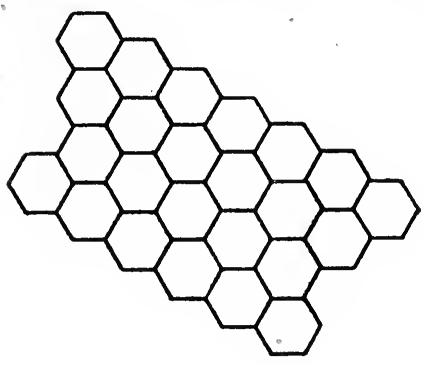

II

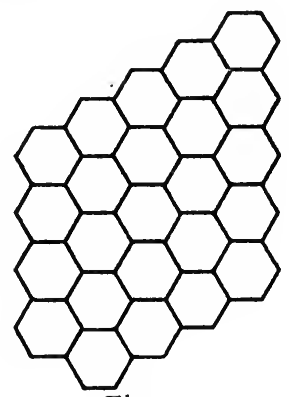

Fig. 53 .

Io boundary compartments and each must occur not less than four times. In the case of assemblage II the colours 3, 6 together must occupy either 8 or 16 boundary compartments and each must occur not less than twice. We have therefore types depending upon these two colours as follows:

\begin{tabular}{|c|c|c|c|c|}
\hline & & & & \\
\hline Colours & 3 & 6 & 3 & \\
\hline & I 2 & 4 & 4 & \\
\hline & IO & 6 & 6 & \\
\hline & 8 & 8 & 4 & \\
\hline
\end{tabular}

\begin{tabular}{rrrr}
\multicolumn{4}{c}{ II } \\
3 & 6 & 3 & 6 \\
\hline I 4 & 2 & 4 & I2 \\
I2 & 4 & 2 & I 4 \\
IO & 6 & 6 & 2 \\
8 & 8 & 4 & 4 \\
6 & IO & 2 & 6
\end{tabular}

III

\begin{tabular}{rrrr}
3 & 6 & 3 & 6 \\
\hline 12 & 4 & 4 & I2 \\
I0 & 6 & 6 & 4 \\
8 & 8 & 4 & 6 \\
6 & 10 & &
\end{tabular}

whilst, in each of the three assemblages, the colours $1,2,4,5$ must each occur an even number of times upon the boundary. 


\section{PART II}

\section{THE TRANSFORMATION OF PART I}

- $\quad$ Kind neighbours ; mutual amity prevails ;

Sweet interchange of rays, received, return'd ;

Enlight'ning, and enlighten'd! All, at once,

Attracting, and attracted! Patriot-like,

None sins against the welfare of the whole;

But their reciprocal, unselfish aid

Affords an emblem of millennial love. The Consolation.

37. In Part I we have had before us sets of triangles, squares, etc., which, as regards a particular set, are all of the same size and shape, but are differently coloured or numbered; and we have seen that a particular set of pieces may be set up into a square, rectangular, hexagonal or other shape so that certain contact laws inside the boundary of the figure are satisfied. For a particular set we have an associated outline inside of which the whole of the pieces are arranged. The form of the boundary of the figure is composed of straight lines and does not alter however the relative positions of the pieces may change. Although the geometry of the figure does not vary, the boundary is differently coloured or numbered for each type of boundary and for each variety of type.

The question now before us is the transformation of the set of pieces so that they will no longer be of the same shape and differently coloured or numbered. It proves to be possible to effect this so that the pieces are of different shapes, and are not differently coloured, numbered or otherwise distinguished. In fact, instead of having a set of pieces of the same shape and differently coloured we construct a set of different shapes but of the same colour.

The boundary of the assembled pieces now varies in shape with each type and variety but is not otherwise distinguished.

Studia prima la scienza, e poi seguitá la pratica nata da essa scienza. LEONARDO DA VINCI.

Suppose that we have two equilateral triangular pieces as in fig. $54 a$ and that the contact system involves a compartment of colour I being adjacent to a compartment of the same colour. 
At present the boundary of the compartment concerned is the straight line $A B$. Is it possible to substitute another boundary which will still allow the two compartment boundaries to lie up against one another?

In fig. $54 b$ bisect $A B$ in $E$ and $O B$ in $D$. Draw $A F$ at right angles to $A C$. Join $D E$ and produce to meet $A F$ in $F$.

Take $A F D B$ as a new compartment boundary in both triangles and it will be found that by rotations of the left-hand triangle clockwise about $B$ and of the right-hand one counter-clockwise about $A$, the two new boundaries will lie perfectly up against one another, as shewn in fig. $54 C$ where $A F D B$ is the common boundary of pieces which are still of the same size and shape.
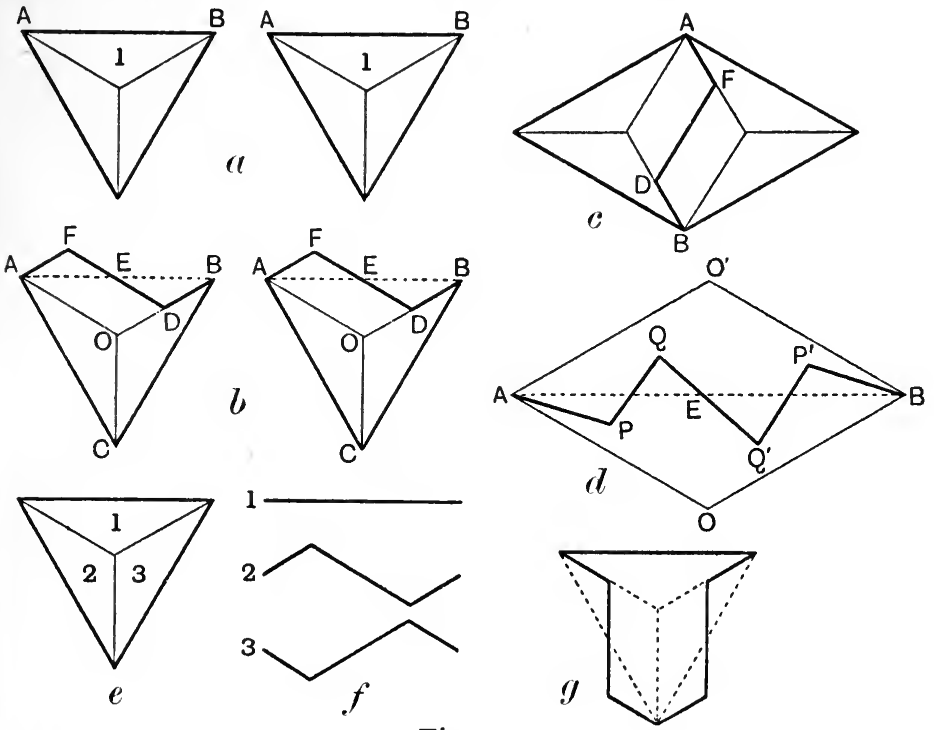

Fig. 54 .

A compartment originally coloured I will always fit as desired after the transformation provided that no compartment of a different colour is transformed in the same way.

It will be noticed that the transformation does not alter the area of the piece and that the colour $I$ is no longer necessary. There is no limit to the number of ways in which the alteration of a compartment boundary can be carried out.

Consider the parallelogram $A O B O^{\prime}$ in fig. $54 d$ formed by two adjacent compartments and any point $P$ inside; also the corre- 
sponding point $P^{\prime}$ found by joining $P E$ and producing it an equal distance to $P^{\prime}$.

Also select any other point $Q$ inside and find the corresponding point $Q^{\prime}$. Then taking $A P Q Q^{\prime} P^{\prime} B$ as the common boundary of the two pieces we see that the two pieces will be of the same size and shape and will fit into one another perfectly.

Any number of such points $P, Q$ may be taken either upon the boundary of the parallelogram or inside of it, and an appropriate compartment boundary will result.

In particular the points may be infinite in number so that a curve is produced by joining them.

It follows that the transformed boundary may be determined in infinite variety.

Suppose then that we have any number of colours and the first contact system in which each compartment is adjacent to one similarly coloured, we are able to select any convenient boundaries to associate with the colours and one of these may be the straight line, the original compartment boundary. The shape of each compartment of each piece may thus be transformed and the pieces themselves given various curious shapes depending upon the choice of compartment boundaries.

The boundary shapes that are found to be the most suitable for the equilateral triangle are not necessarily the best for the square, hexagon, etc.

To give in this place one example, suppose that fig. $54 e$ is one of a set of triangles, we may associate with the colours the boundaries as in fig. $54 f$ transforming the piece to the colourless shape of fig. $54 g$.

If, in the coloured or untransformed set of triangles, the type be such that the colour I is to monopolise the boundary, it will not be changed because the boundary associated with the colour I has not been altered. But if the type of boundary be other than that which has been specified the boundary of the setting will exhibit some of the new compartment boundaries that have been introduced. Thus the pieces instead of setting up always into the same shape, differently coloured or marked, set up into different colourless shapes.

This circumstance adds much to the interest of the pastime of fitting the pieces into shapes which have been previously determined, by the methods of Part I, to be possible. 
38. In the case of the second kind of contact we have, say, a colour I lying invariably up against a colour 2 and, whatever may be the number of colours, we must arrange such a transformation of compartment boundaries that the colour I will lie up against colour 2 but will not lie up against any of the colours I, $3,4,5, \ldots$.

Taking, as before, the equilateral triangle as an illustration, we must arrange for the compartment boundary of colour I to fit into the boundary we associate with colour 2 , taking care that the two boundaries differ from one another.

If to compartment I we add on a projecting piece $A K B$ (fig. $55 a$ ), equal and similar to $A O B$ so that the angle $K A C$ is a right angle, and cut out the compartment 2 altogether, it is clear that the projecting piece $A K B$ will exactly fit into the recess $A J B$, and that the combined area of the transformed triangles will not be changed.

The figure $55 b$ shews the piece $C A K B$ fitting the piece $A K B C^{\prime}$. The conditions are satisfied because the compartment boundaries $A K B, A J B$ are quite different.

We must now discover the most general transformation of this nature. It is clear that the area of each triangle (but not the combined area) will usually be altered by the transformation.

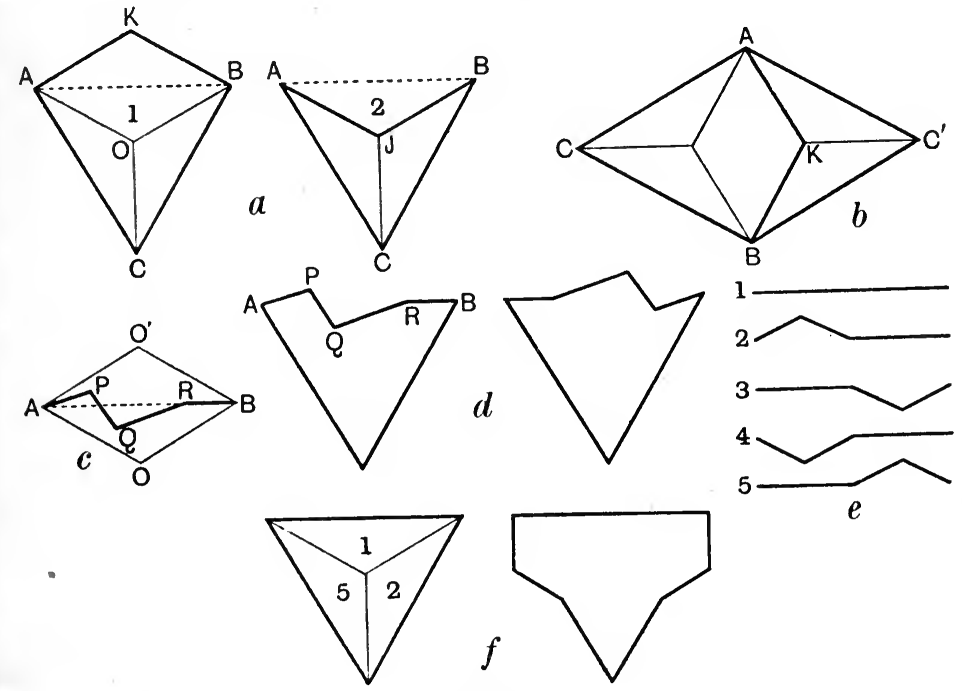

Fig. 55 . 
As will appear, the area of each triangle is only exceptionally unaltered.

Take, as before, the parallelogram of the compartments $O A O^{\prime} B$, fig. $55 c$. We can select in the area any number of points we please, which may lie if we like either upon the boundaries of the parallelogram or upon $A B$, and join them by straight lines. We shall then have suitable boundaries to the compartments as shewn in fig. $55 d$.

A necessary precaution is to avoid the kind of symmetry about the middle point of $A B$ that always exists in transformations connected with the first kind of contact.

When the number of points selected $P, Q, R, \ldots$ is infinite we are led to boundaries wholly or partially curved.

We take separate pairs of compartment boundaries for the different associated pairs of colours in the contact system.

Each compartment of each triangle is thus transformed*.

As an example, suppose that we have five colours and that the contact system is $C_{\mathrm{I}, 2,2}$ :

$\begin{array}{lll}\text { I } & \text { adjacent to I } \\ 2 & " & 3 \\ 4 & " & 5\end{array}$

we may select the boundaries as in fig. $55 e$, obtaining the transformation shewn in fig. $55 \mathrm{f}$.

We are now in a position to transform the pieces of the various pastimes described in Part I. We can also transform any type of boundary as soon as we have determined the variety of type that is to be employed. We have then the problem of fitting the pieces into the boundary, which usually demands both patience and thought.

In the transformation of the pieces there is ample scope for taste and judgment. The simplest straight-line forms should generally be chosen as it is easier to construct them accurately than those which involve circular arcs. Very acute angles should be avoided as far as possible so that the pieces may not be too fragile. This presents a real difficulty which however may be practically surmounted by the exercise of ingenuity.

* It is not essential for the compartment boundary to lie entirely within the parallelogram of compartments in this or in the case of the first kind of contact. The condition however is in inost cases advisable and may be recommenderl. 
Quel che è nuovo è sempre bello.

Goldoni.

39. The transformation of the 24 pieces of Pastime no. 2, four colours on a triangle with repetitions allowed, for the contact system $C_{\mathrm{I}, \mathrm{I}, \mathrm{I}, \mathrm{I}}$ may be carried out in the following manner. Taking the boundaries as in fig. 56 we get the pieces as in fig. 57. In fig. 58 these are shewn assembled inside a boundary of regular hexagonal shape.

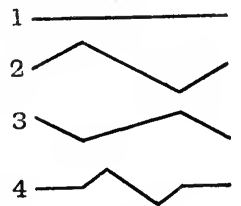

Fig. 56.
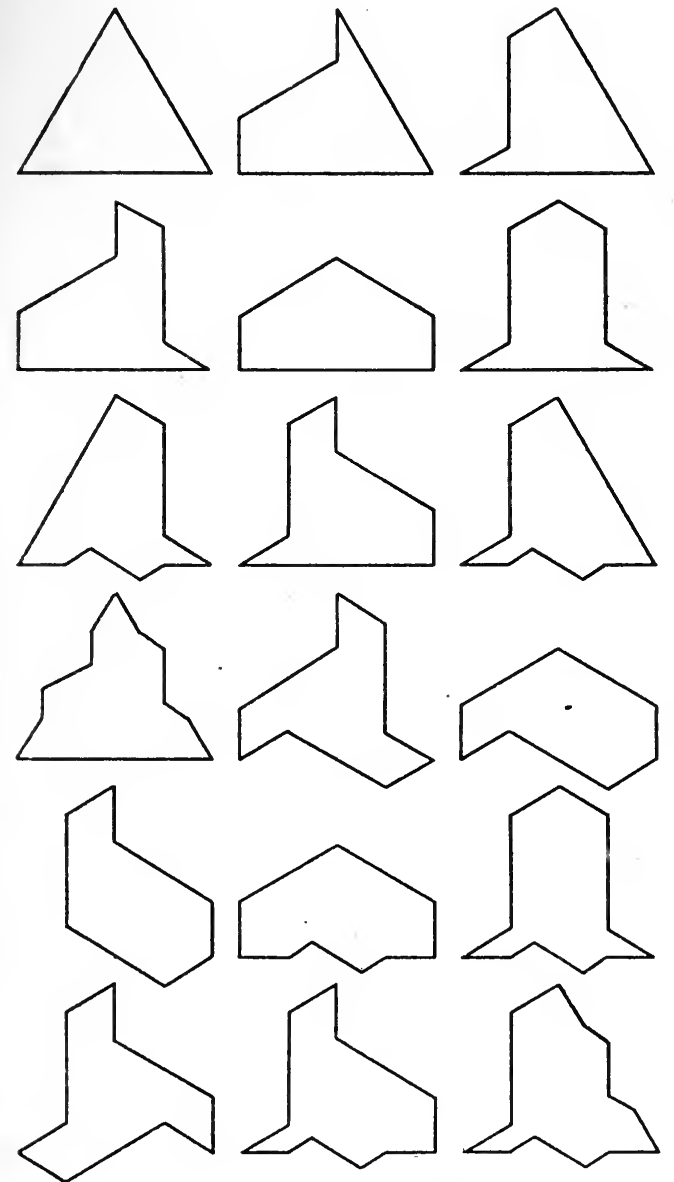

Fig. 57 .
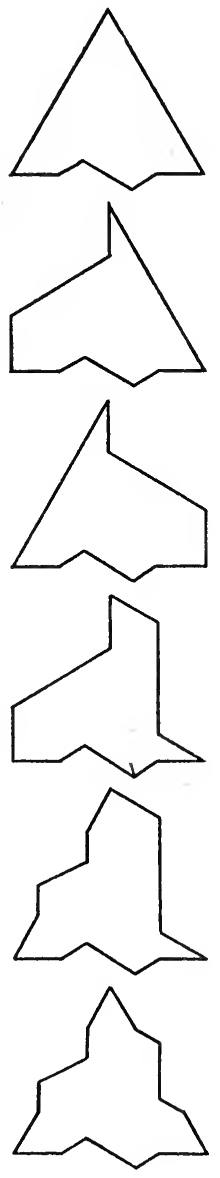
Nor think thou seest a wild disorder here: Through this illustrious chaos to the sight, Arrangement neat, and chastest order reign. The path prescribed, inviolably kept Upbraids the lawless sallies of mankind.

The Consolation.

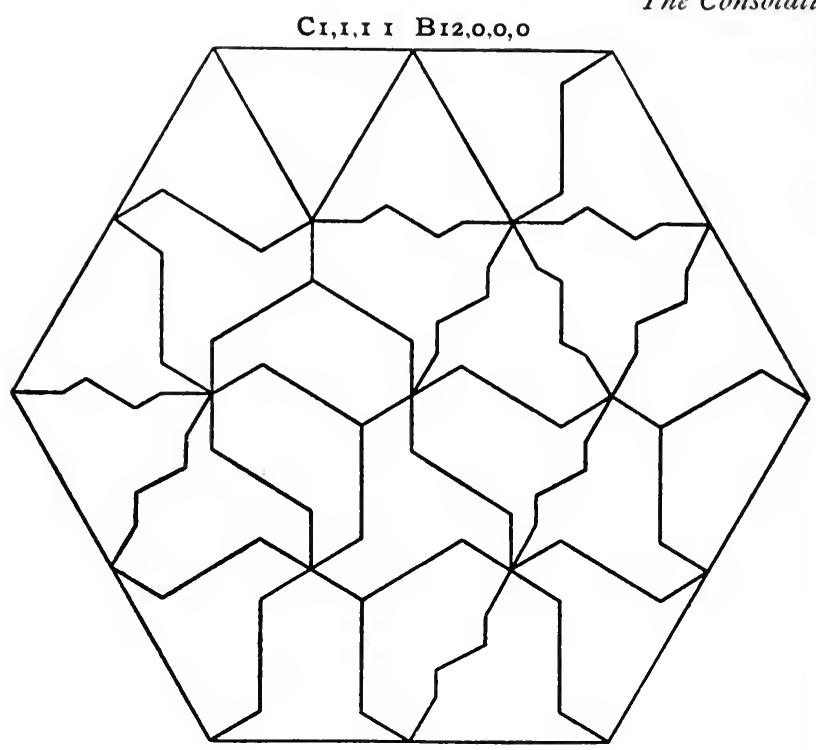

Fig. 58 .

$\mathrm{Nil}$ fuit unquam

Sic impar sibi.

HORACK.

40. For each contact system transformation leads to a different set of pieces. Thus for the system $C_{\text {I,I, }}$ or I to I, 2 to 2,3 to 4 , we choose the boundaries as in fig. 59 .

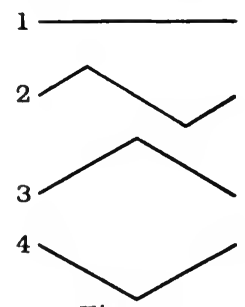

Fig. 59.

The piece each of whose compartments is coloured 4 vanishes with the transformation and we obtain the twenty-three pieces shewn in fig. 60. 
Bring then these blessings to a strict account;

Make fair deductions; see to what they 'mount' :

How much of other each is sure to cost;

How each for other oft is wholly lost.

Pope, Ess. Man, IV. 270.
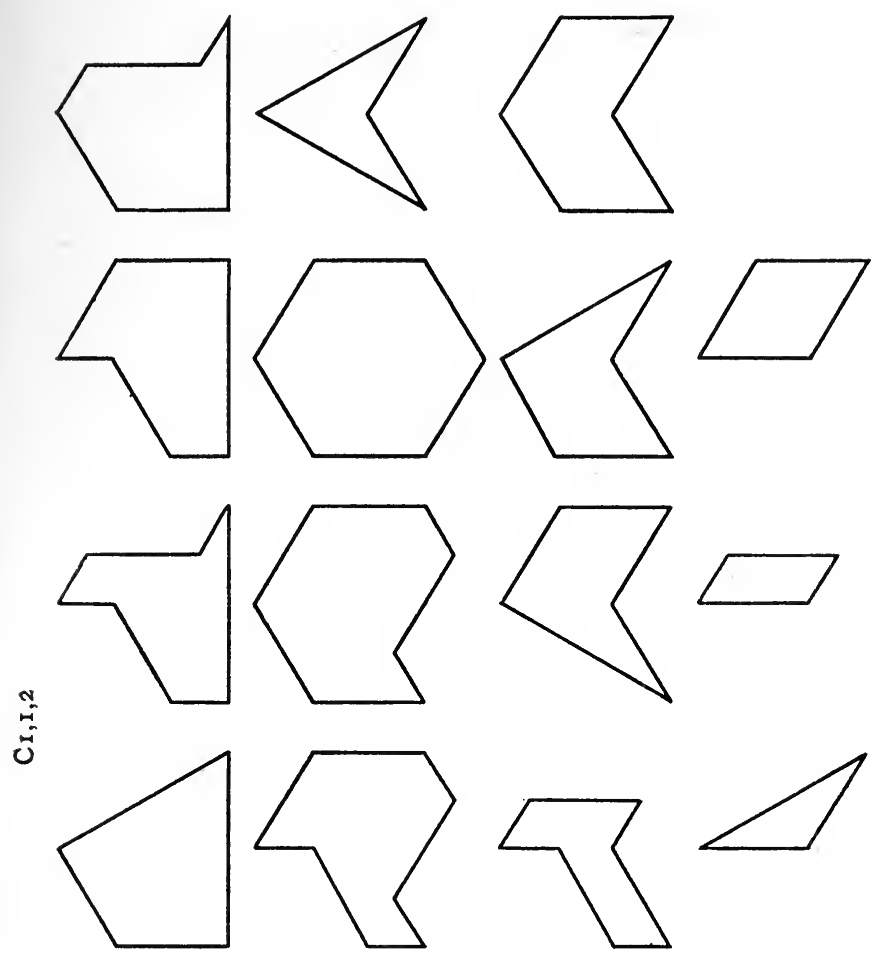

8
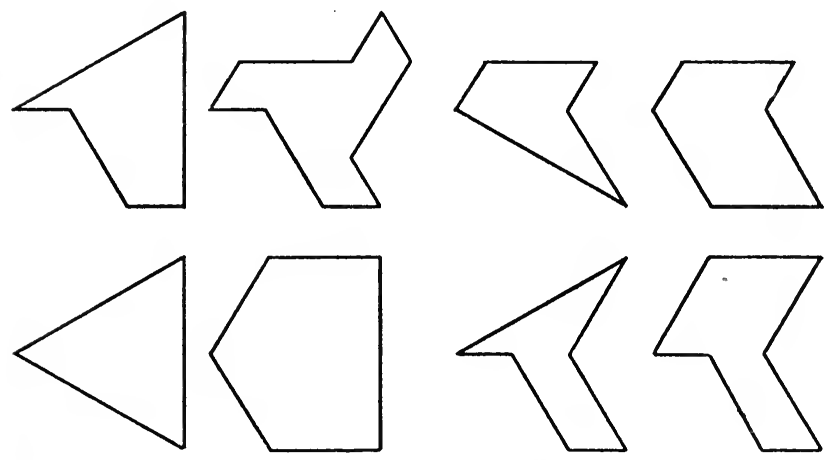
The assemblage of the pieces according to fig. $6 \mathrm{I}$, which is the figured diagram of Part I, is shewn in fig. 62.

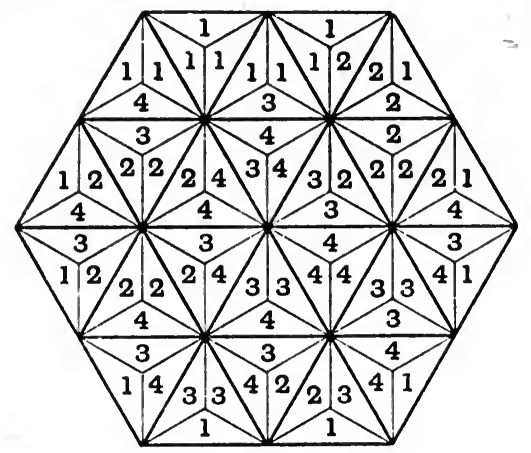

Fig. 6I.

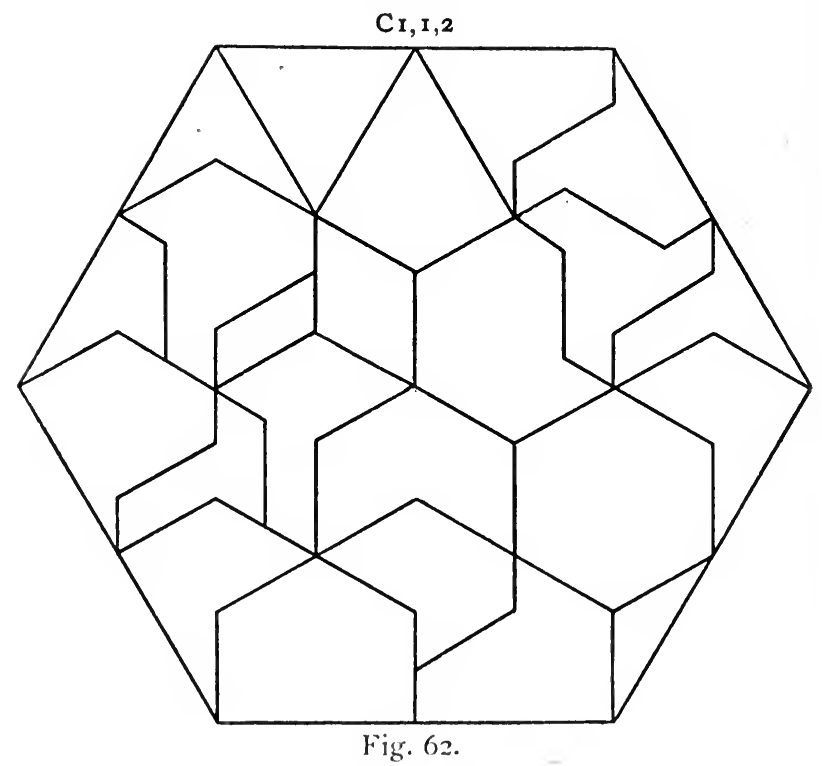

Sic ludus animo debet aliquando dari, Ad cogitandum melior ut redeat tibi.

PHAEDr. Fab. 111. 14.

41. For the third contact system $C_{2,2}$ which is I to 2 , 3 to 4 we select for the colours as in fig. 63 ; one piece again vanishes for the transformation and we are left with the twentythree pieces shewn in fig. 64 . 


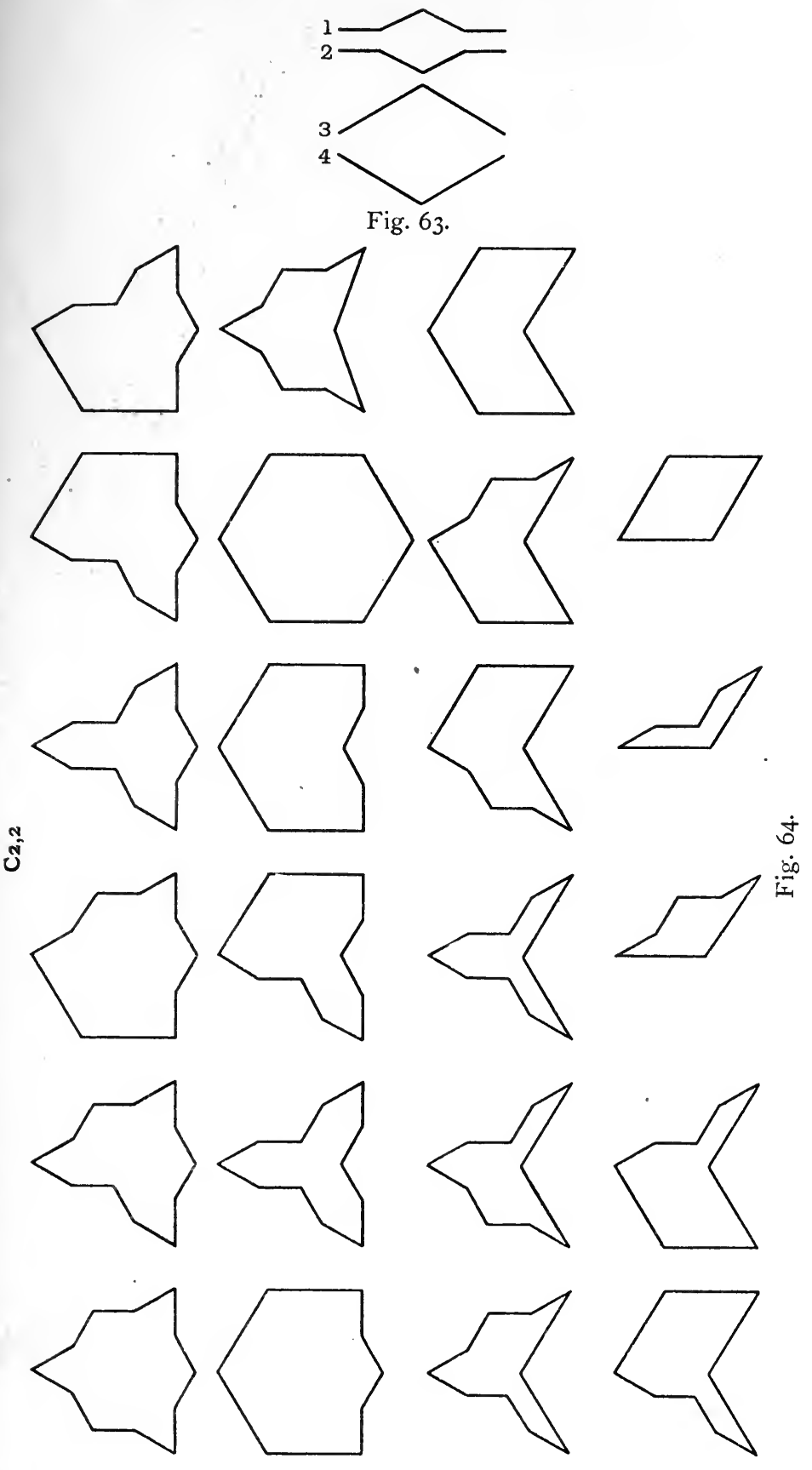


In fig. 65 the pieces are assembled according to the diagram C2,2 B6,6,0,0 of Part I.

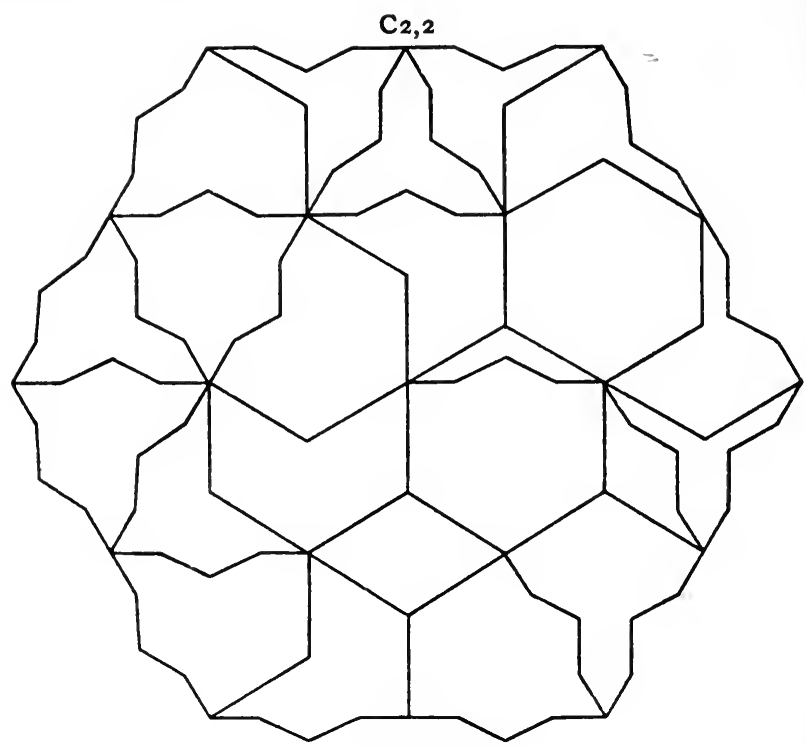

Fig. 65.

Behold, said Pas, a whole dicker of wit.

Pemiroke, Arcadia, p. 393.

42. For the transformation of the ro-piece set on the contact system $C_{1, \mathrm{I}, \mathrm{I}}$ we may take the colours as in fig. $66 a$
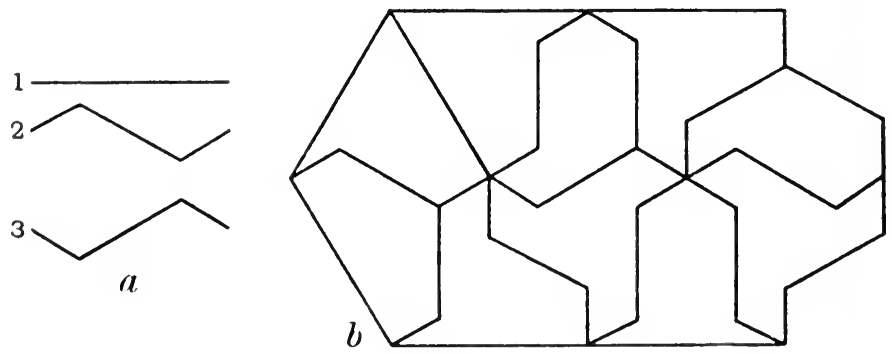

Fig. 66.

and we find for the first figured assemblage $C_{1,1,1} B_{1}, \mathrm{I}, 6$, the fig. $66 b$ shewing the shapes of the transformed pieces and of the boundary for the particular case. 
43. Skurffe by his nine-bones swears, and well he may, All know a fellon eate the tenth away.

HERRICK.

Similarly for the contact system $C_{2}$, I, taking the colours as in fig. $67 a$ for the first figured assemblage $C_{2, \mathrm{I}} B_{4,4,0}$ we have fig. $67 b$ shewing the shapes of the transformed pieces and of the particular boundary. One piece vanishes.

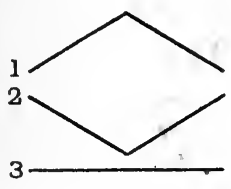

$a$

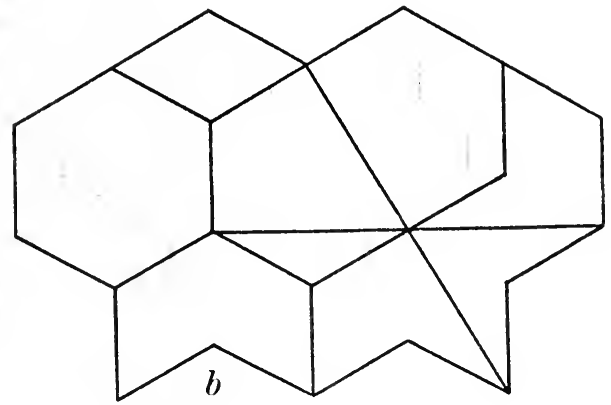

Fig. 67.

44. For the remaining contact system $C_{\mathrm{I}, 2}$ we may take the colours as in fig. $68 a$, and for the first figured assemblage $C_{1,2} B 5,3,0$ we obtain fig. $68 b$ giving the shapes of the pieces which differ from those in the preceding case. Moreover there are ten of them instead of nine.
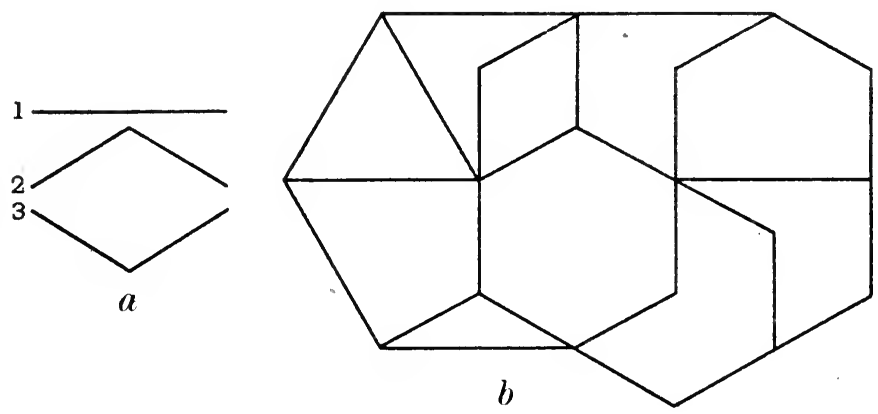

Fig. 68.

45. For the transformation of the I3-piece set we may for

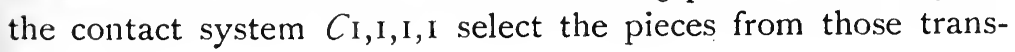


formed from the complete set of 24 for the same contact system. The second figured arrangement is then as in fig. 69 .

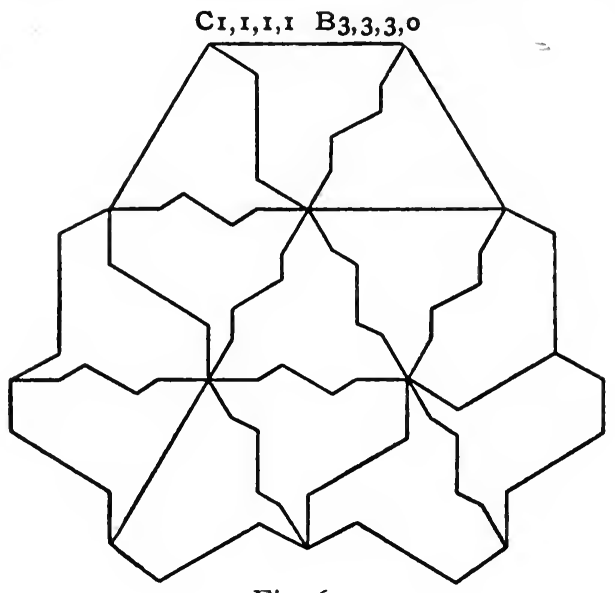

Fig. 69.

46. For the contact system $C_{\mathrm{I}, 2, \mathrm{I}}$, viz. I to I, 2 to 3,4 to 4 , we may take the colours as in fig. $70 a$; we have then fig. $70 b$.

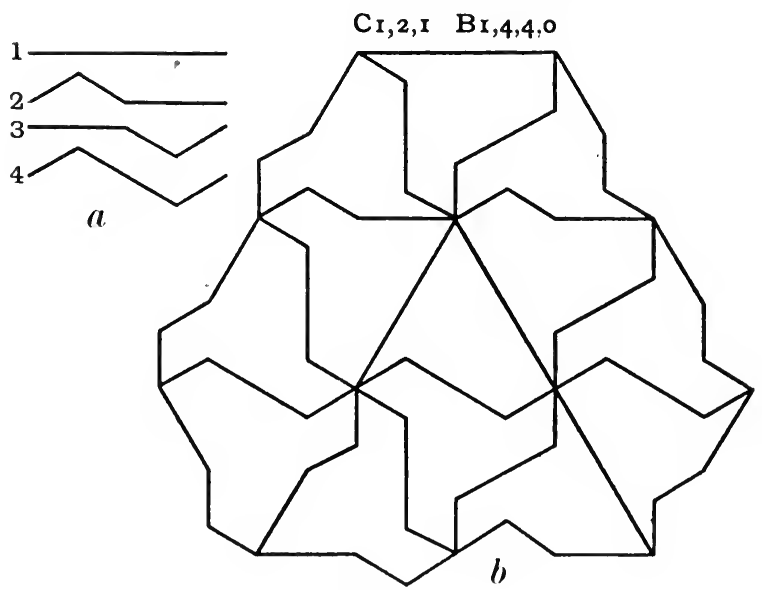

Fig. 70 .

Affirm'd the trigons, chopp'd and changed.

Hudib. II. iii.

47. Coming next to the five-colour triangle we choose, for

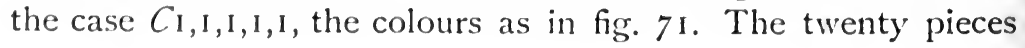


THE TRANSFORMATION OF PART I

are as in fig. 72 and the assemblage for $C_{\mathrm{I}, \mathrm{I}, \mathrm{I}, \mathrm{I}, \mathrm{I}} B_{\mathrm{I}} 2, \mathrm{O}, \mathrm{O}, \mathrm{O}, \mathrm{O}$ is as in fig. 73 .

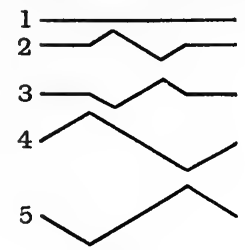

Fig. 7 I.
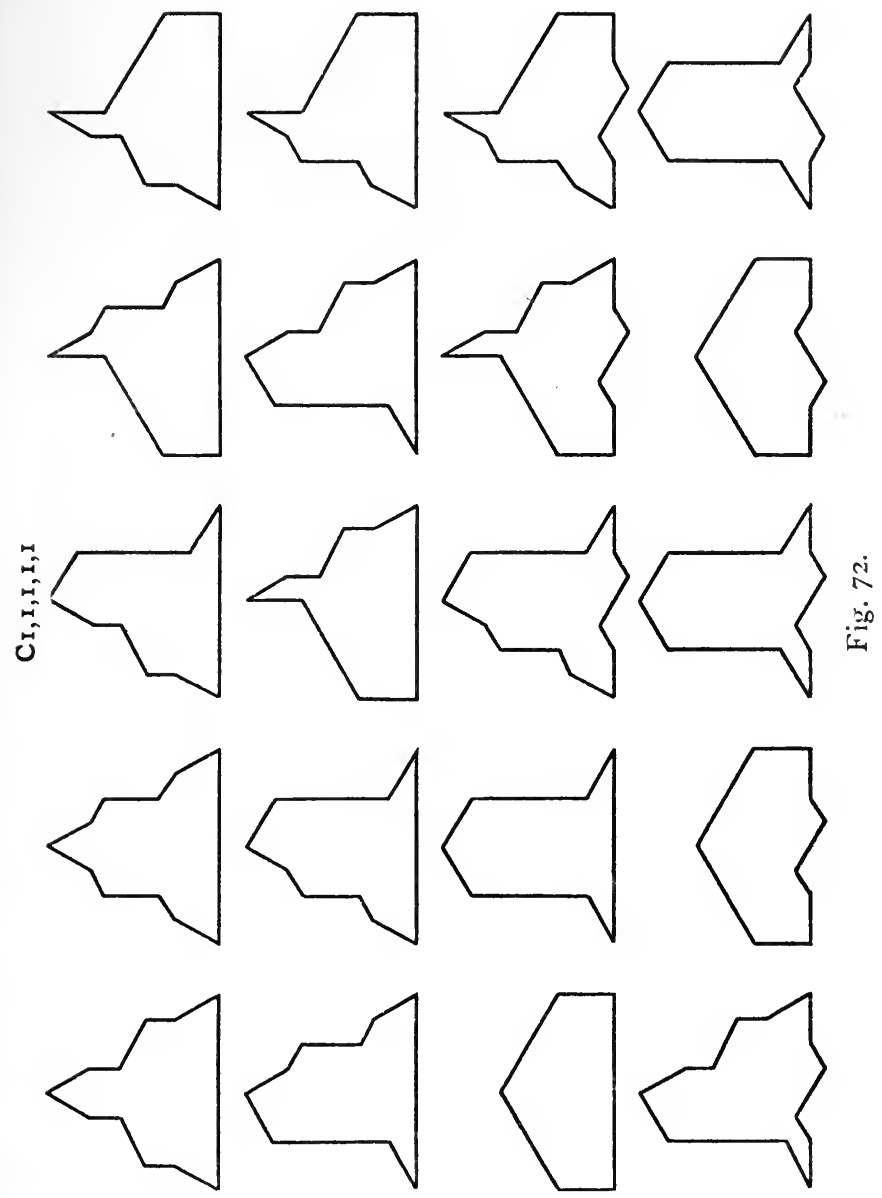


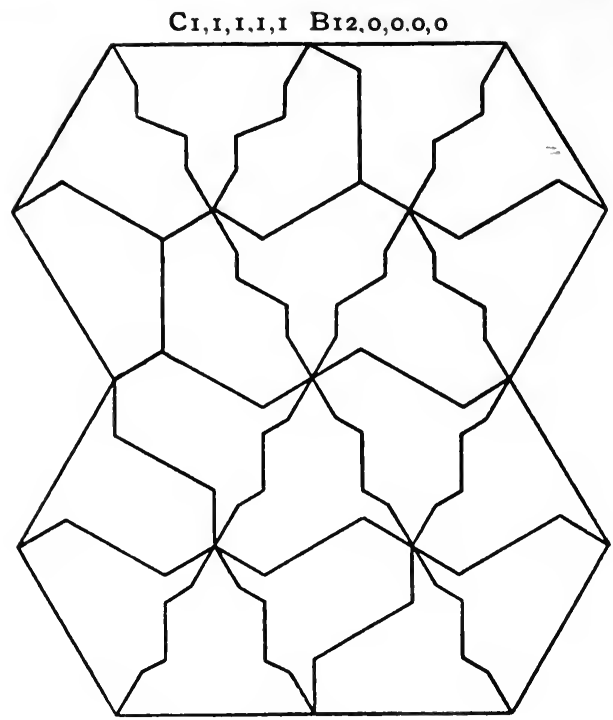

Fig. 73 .

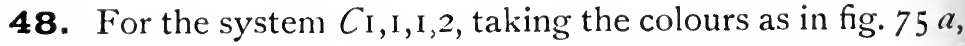
the pieces are as in fig. $75 b$, the assemblage as in fig. 74 .

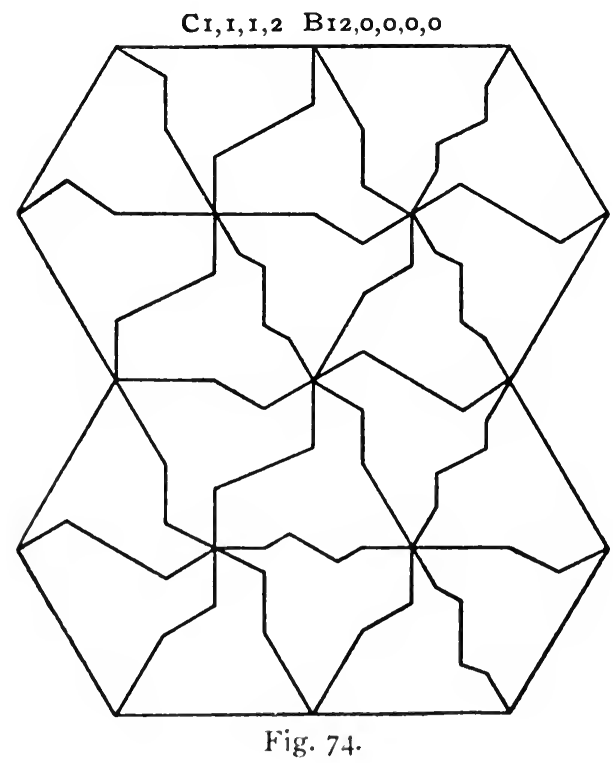



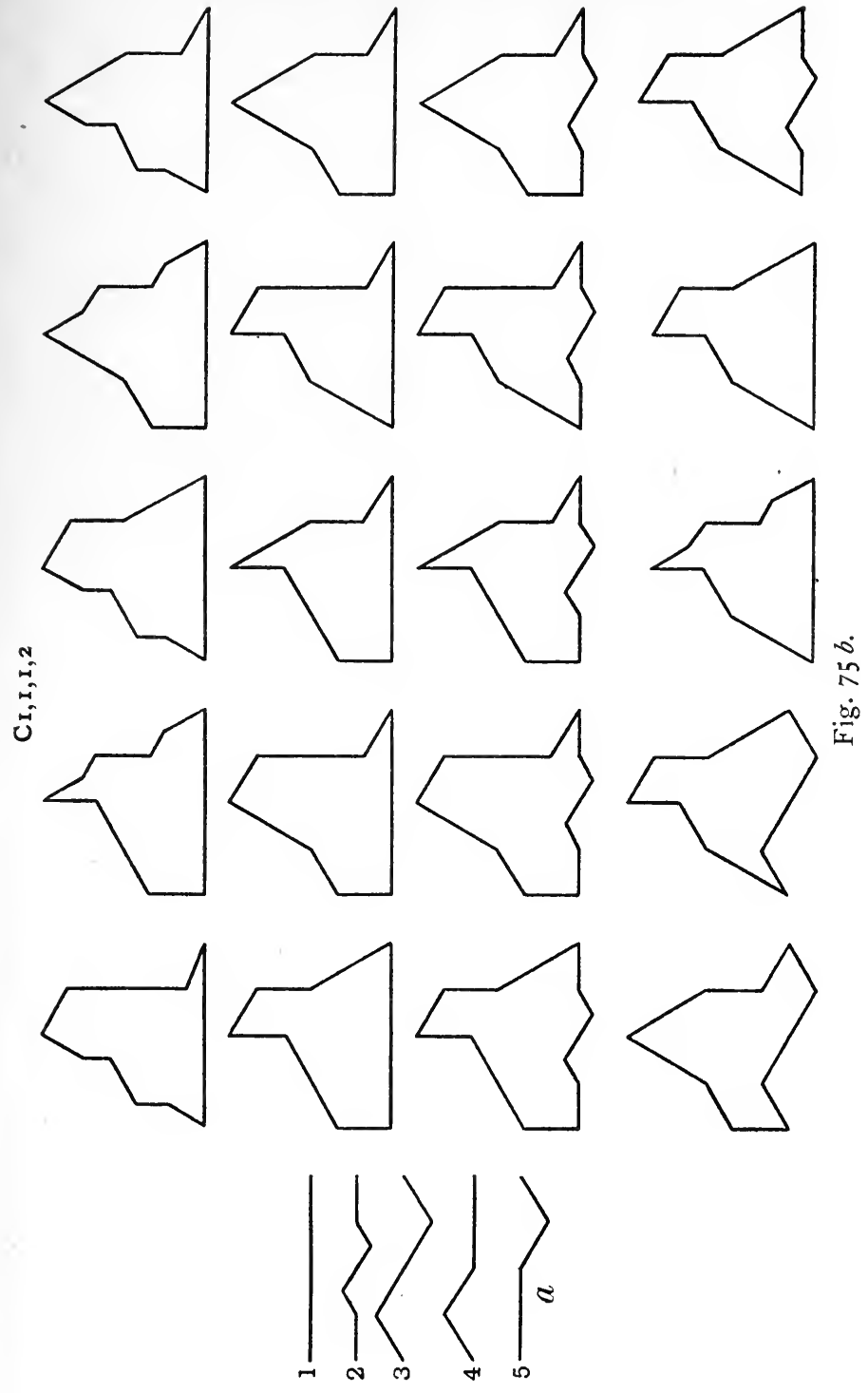

M. P. 


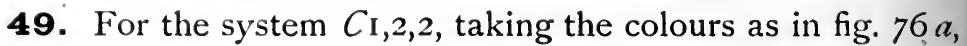
the pieces are as in fig. 76 , and the assemblage for $C_{\mathbf{I}, 2,2}$ $B$ I $2,0,0,0,0$, as in fig. 77 .
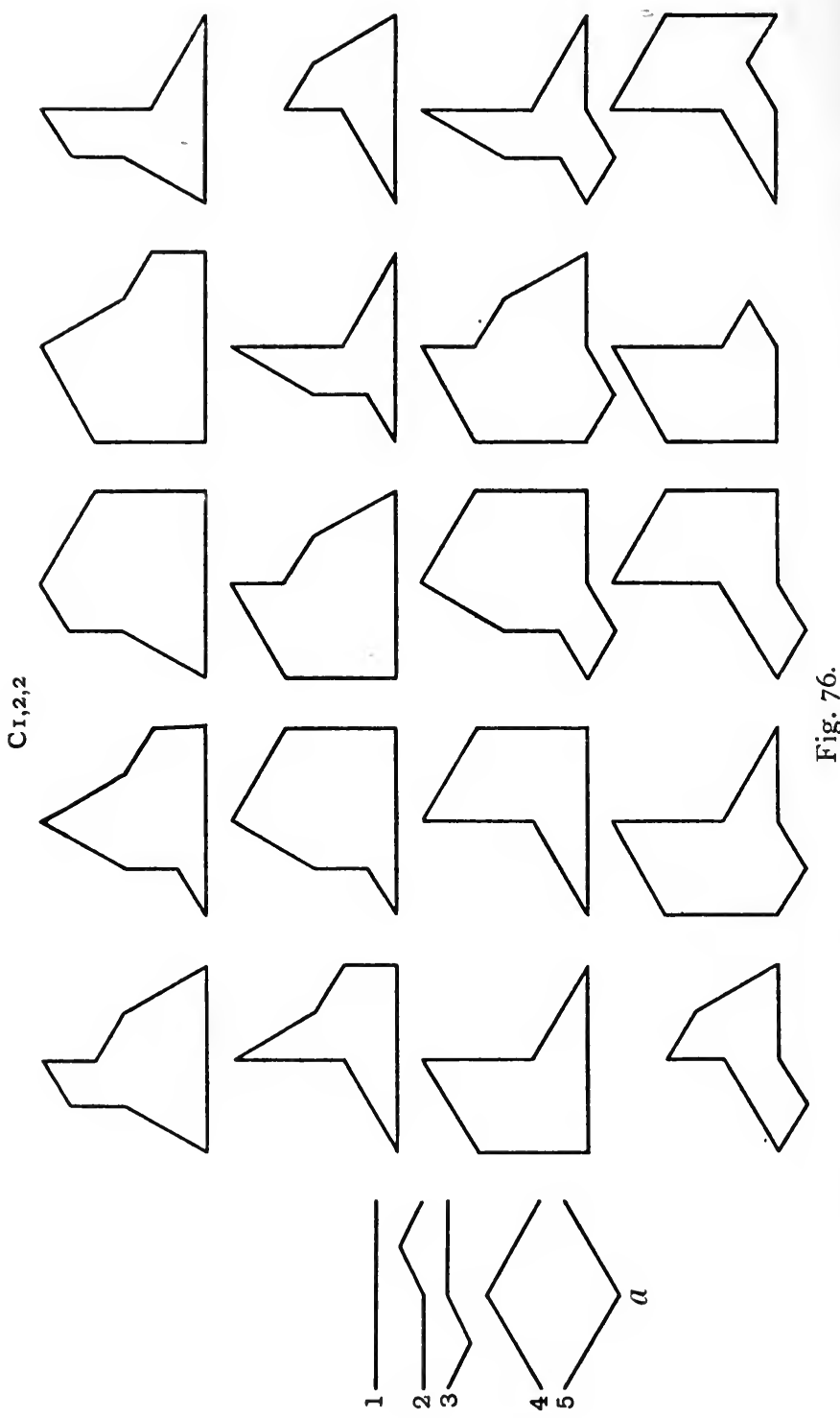


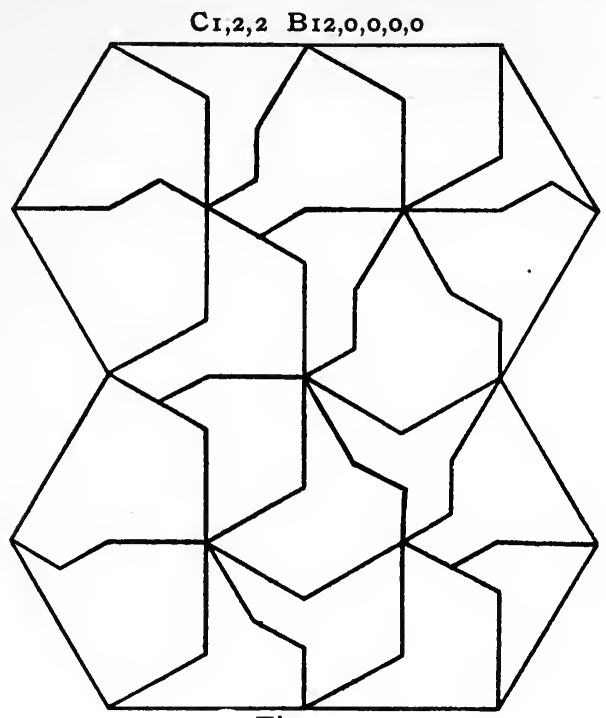

Fig. 77 .
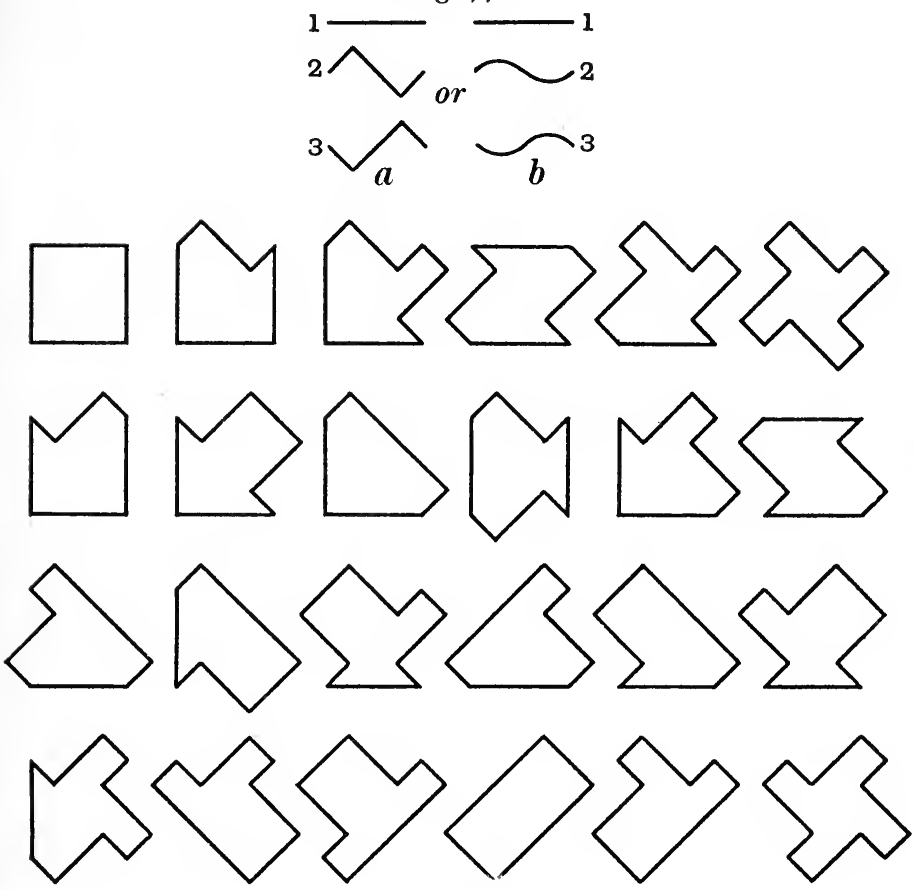

Fig. 78.

(See page 68.) 
Has matter more than motion? Has it thought, Judgment and genius? is it deeply learn'd In mathematics?

The Consolation.

50. In the case of pieces based upon the square the compartment boundaries must be modified so as to bear a convenient relation to the angles of the parallelogram of the compartments. The parallelogram is here a square and an angle of $45^{\circ}$ should be often in evidence. For the contact system $C_{\text {I, I, I suitable }}$ boundaries for the 24-piece set may be as in fig. $78 a$ or $b$, p. 67 .

The system of pieces for the first of these is given in fig. 78 .

For any given boundary defined by colours as in Part I, the figure into which the pieces may be assembled can be drawn.

Thus, as an example, if we take a type and variety such that the colours 2, 3 occur alternately we find the figure $79 b$ and so on. In each case, we make the boundary transformation.
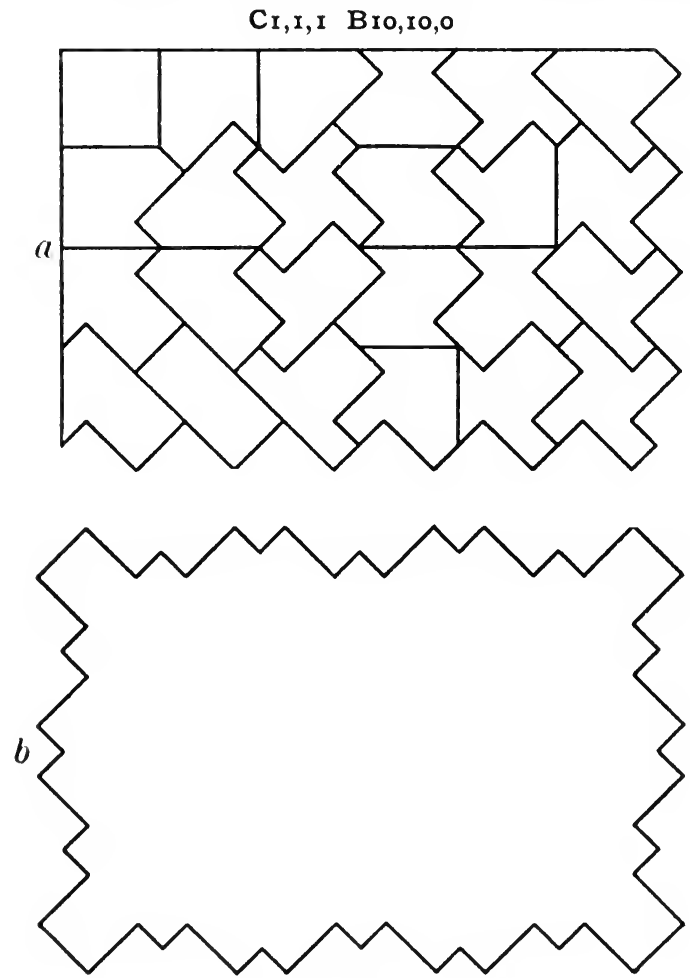

Fig. 79 . 
A great many symmetrical figures can usually be drawn, with the exercise of a little ingenuity, for any contact system.

The assemblage for $C_{\mathrm{I}, \mathrm{I}, \mathrm{I}} B \mathrm{IO}, \mathrm{IO}, \mathrm{O}$ is given in fig. $79 a$.

After mutch counsayle and great tyme contrived in their several examinations.

Pal. of Pleas. D. d. 2.

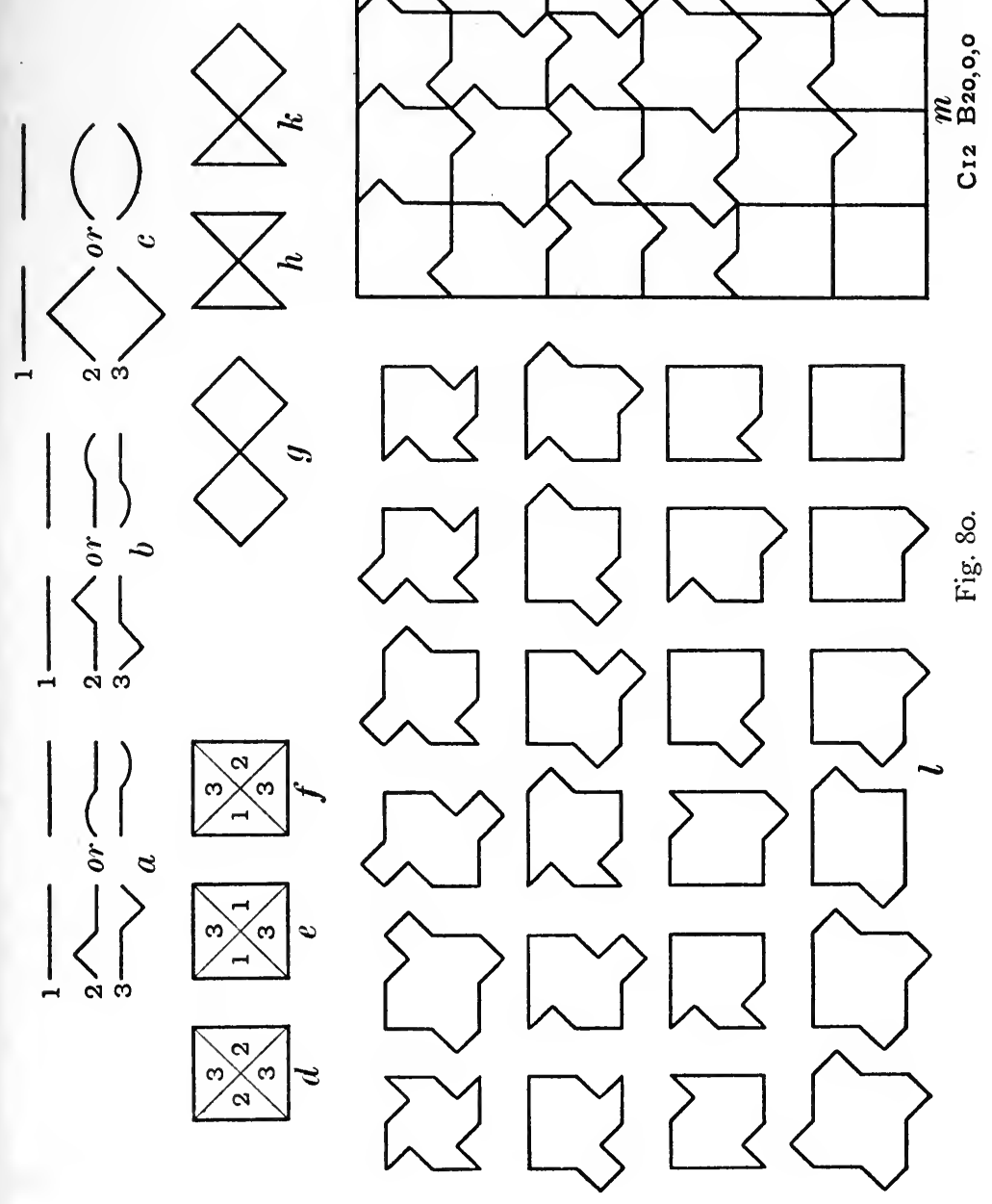


The power and corrigible authority of this lies in our wills.

Othello, 1. iii.

51. With the contact system $C_{\mathrm{I}, 2}$, viz. I to I, 2 to 3 , we have a good choice for the compartment boundaries; we may take, for example, the colours as in fig. $80 a, b, c$.

In the last straight line system of boundaries the piece, which has every compartment coloured 3 , vanishes so that the set is one of 23 pieces.

A reference to the boundary types of Part I shews that the set will fit into a large number of symmetrical boundaries.

It should be noted that this 23-piece set is really not suitable because the pieces in fig. $80 d, e, f$ transform into those in fig. $80 \mathrm{~g}, h, k$, each of which, consisting of two pieces meeting at a point, cannot be handled when constructed in cardboard or wood, but only in diagrams.

These pieces must always be avoided.

The twenty-four pieces are as in fig. $80 \mathrm{l}$ and for the assemblage $C_{\mathrm{I}, 2} B 20,0,0$ we have fig. $80 \mathrm{~m}$.

52. In the case of the 20-piece set of Pastime no. 20 and the contact system $C_{\mathrm{I}, \mathrm{I}, \mathrm{I}}$ we have only to discard the pieces in fig. $8 I$ and then to transform the coloured boundaries in the

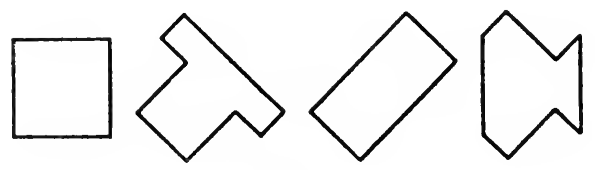

Fig. 8I.

usual manner to obtain a set of figures into which the pieces may be assembled.

53. For the contact system $C_{\mathrm{I}, 2}$, viz. I to I, 2 to 3 , we discard from the corresponding set of 24 pieces those shewn in fig. 82 and proceed in the same way.

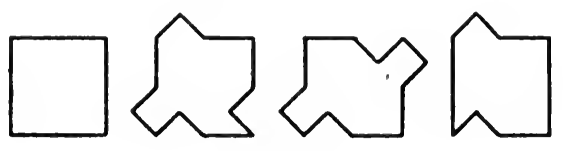

Fig. 82 . 
54. For the contact system $C_{2}$, I, viz. I to 2,3 to 3 , we may discard from the set of 24 pieces corresponding to $C_{\mathrm{I}, 2}$ the four shewn in fig. 83 and take the correspondence between colour and boundary to be that shewn in the same figure.
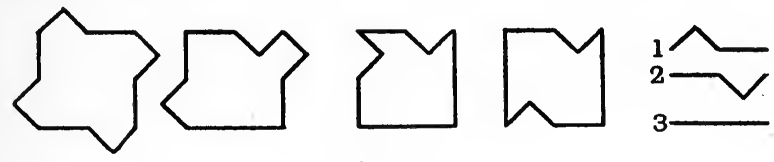

Fig. 83 .

The reader will find the 20-piece set very interesting but the pieces appear to be difficult to assemble, compared with some of the other sets.

Nihil tam difficile est quin quaerendo investigari possiet.

TERENCE.

55. The I6-piece set transformed for the contact system $C_{\mathrm{I}, \mathrm{I}, \mathrm{I}}$ is shewn in fig. 84 made up into a square of boundary type $B$ I $6,0,0$.

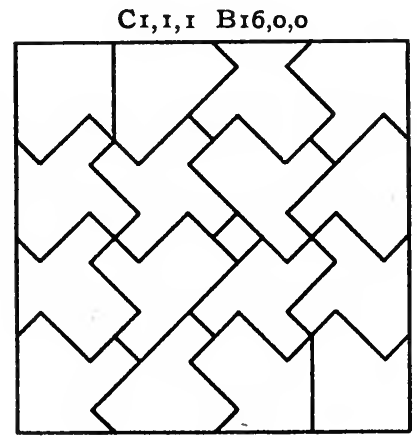

Fig. 84 .

These same pieces may be fitted into a number of symmetrical boundary lines as will be evident to the reader on comparison with the colour schemes of Part I.

56. The reader will probably have no difficulty in dealing with the contact system $C_{2}, \mathrm{I}$, viz. I to 2,3 to 3 . The author has not particularly examined it, but he recommends it with confidence.

Sempre avviene

Che dove men si sa, più si sospetta.

MaChiaVELLI. 
57. He has however put the contact system $C_{\mathrm{I}, 2}$, viz. I to I, 2 to 3 , through much experimental work, and will deal with it in some detail.

He transforms through the correspondence given in fig. $85 a$.

It is shewn in the form of I 5 pieces since the I6th piece derived from fig. $85 b$ vanishes, but it still must be regarded as a I6-piece set forming up into a square $4 \times 4$.

The assemblage for $C_{1,2} B \mathrm{I} 6,0,0$ is given in fig. $85 c$.

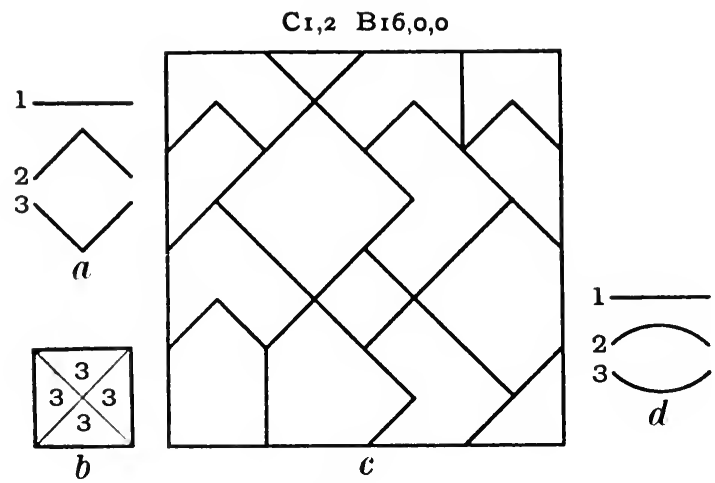

Fig. 85 .

Fifteen symmetrical boundaries, out of a large number that may be constructed, are shewn in figs. 86,87 and 88 , inside each of which the same pieces may be assembled.

\section{Much design}

Is seen in all their motions, all their modes:

Design implies intelligence and art.

The Consolation.

And soo they thre departed thens and rode forth as faste as ever they my3t tyl that they cam to the forbond of that mount.

Morte d'Arthur, I. I 39 .

For the transformation which has the correspondence of fig. $85 d$ the pieces are shewn assembled in a square and three diagrams of boundaries are also given in fig. 89 .

Mark how the labyrinthian turns they take, The circles intricate and mystic maze.

The Consolation. 

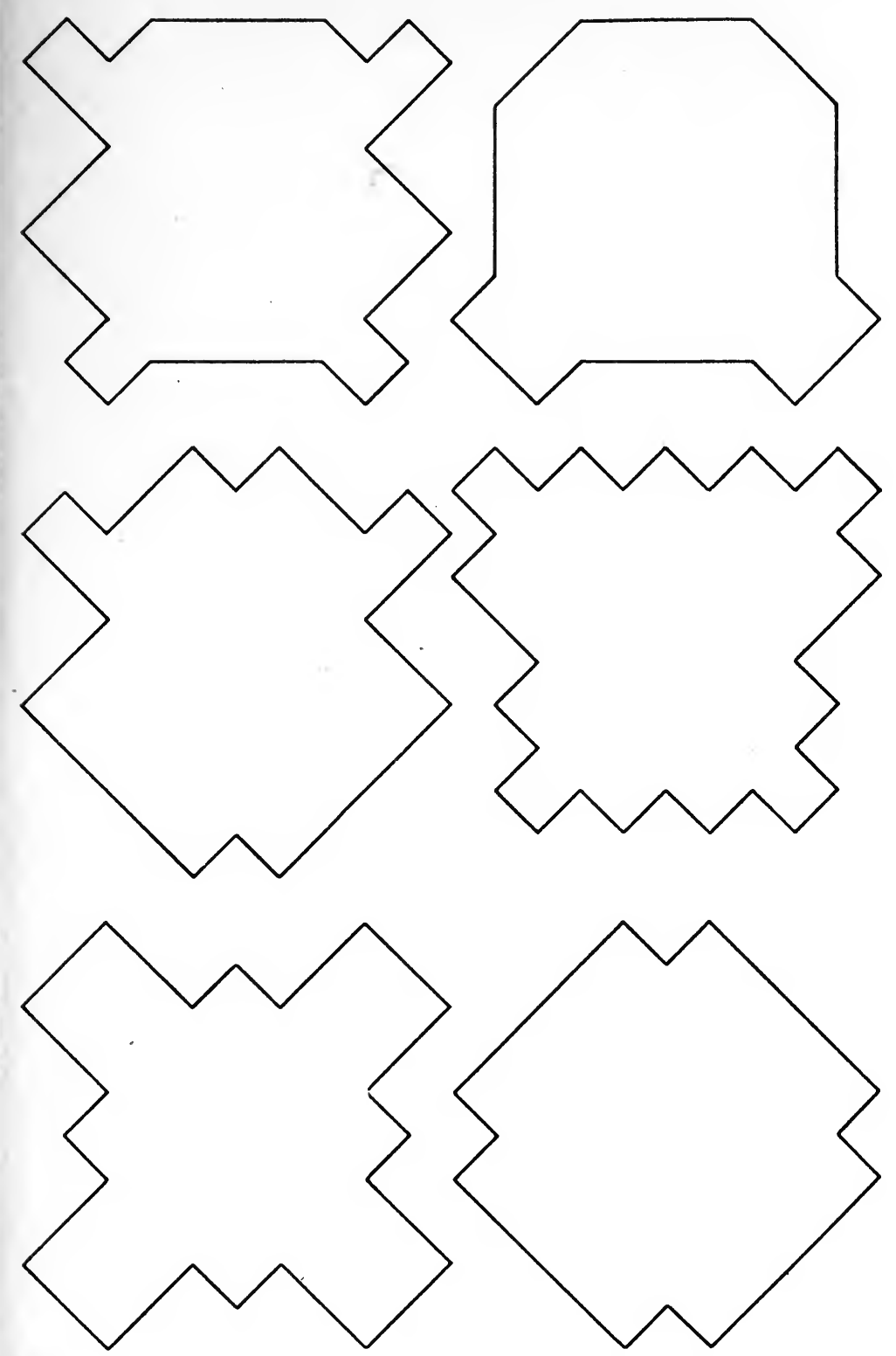

Fig. 86. 

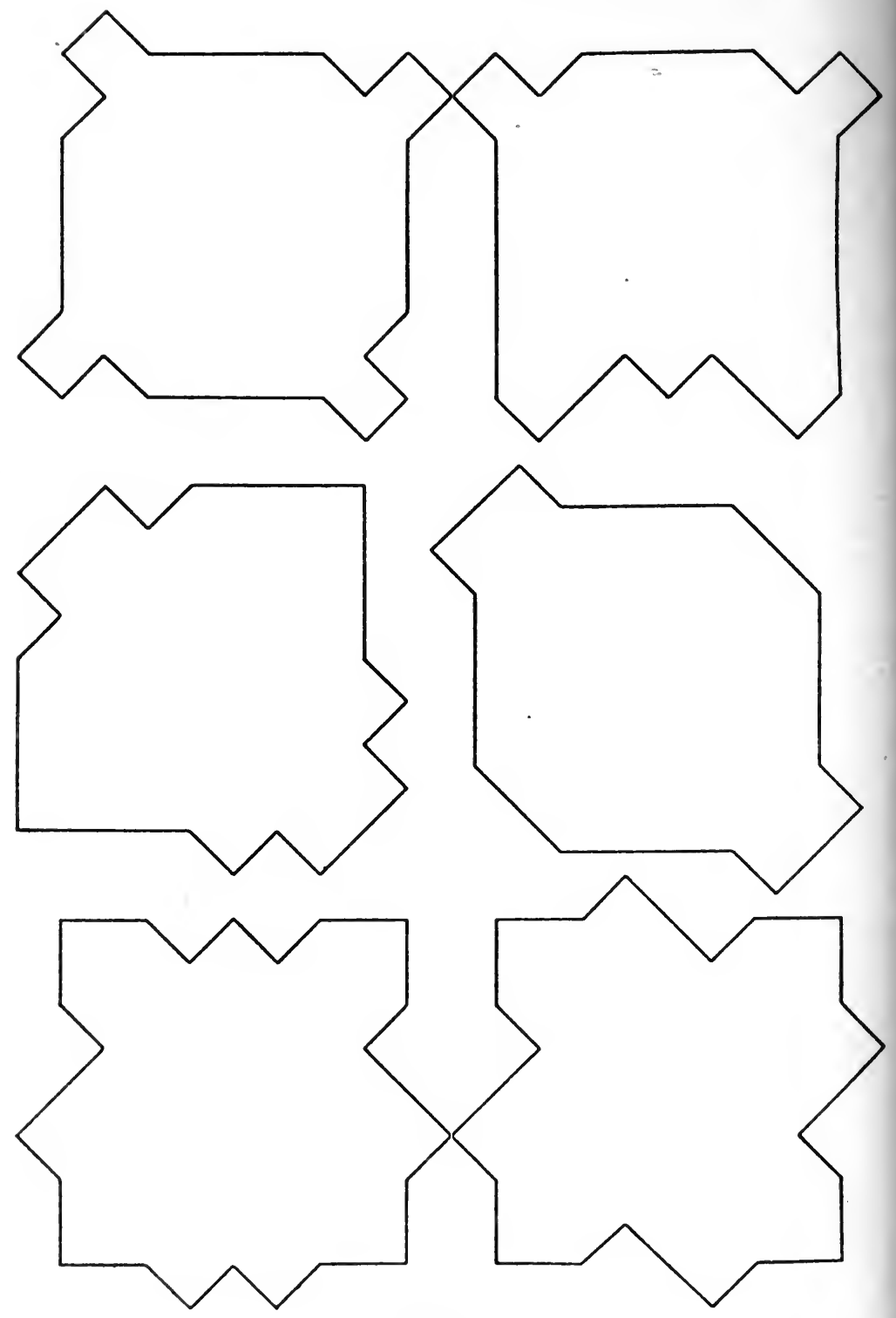

Fig. 87. 


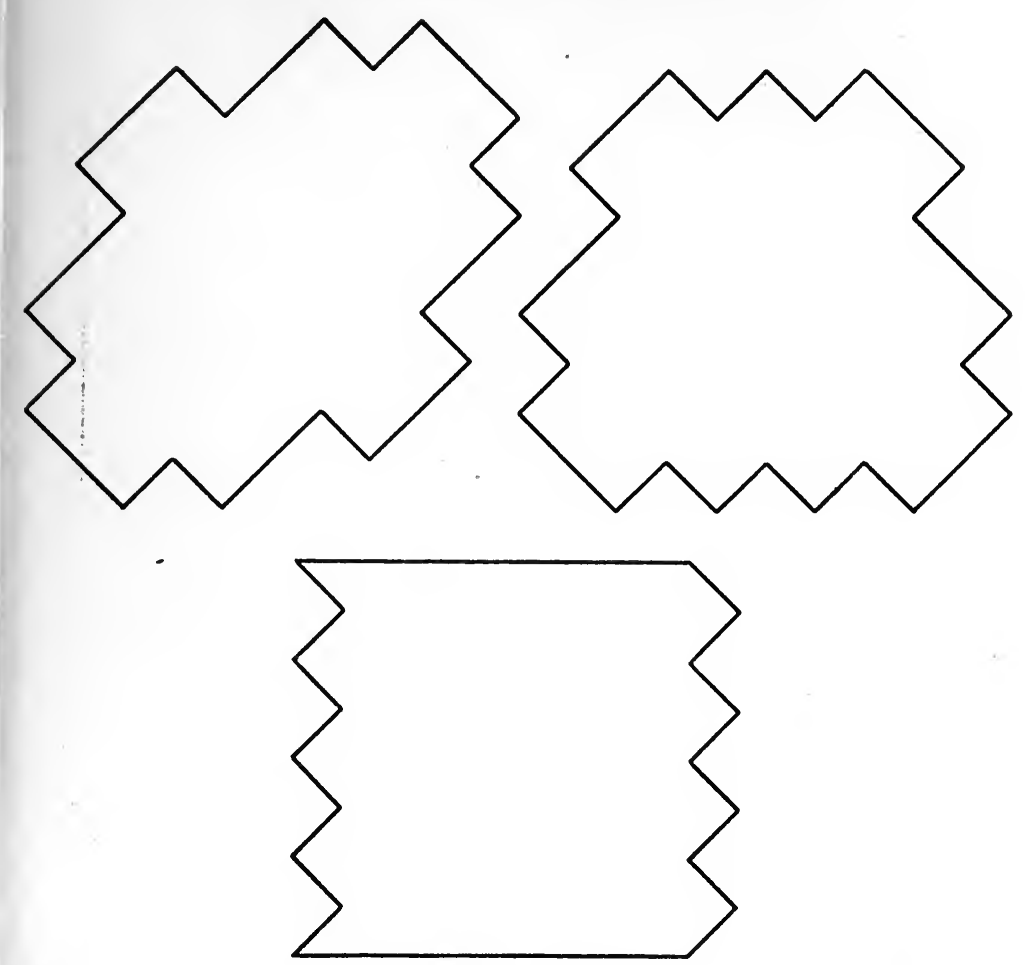

Fig. 88 .

The I5-piece set for the contact system $C_{\mathrm{I}, \mathrm{I}, \mathrm{I}}$ is shewn transformed and put up into the rectangle of fig. 90 and other boundaries for the same pieces are shewn in fig. 9I.

For the contact system $C_{\mathrm{I}, 2}$ we have similar results as in fig. 92 .

For the 9-piece set we may, for the system $C_{\mathrm{I}, \mathrm{I}, \mathrm{I}}$, transpose to the set in the two upper rows of fig. 93 and for the system $C_{\mathrm{I}, 2}$ to the set in the two lower rows of the same figure.

For the system $C_{\mathrm{I}, \mathrm{I}, \mathrm{I}}$ a symmetrical boundary $B 8,2,2$ is shewn in fig. 94 .

For the system $C_{1,2}$ symmetrical boundaries are shewn in fig. 95 . 
76

NEW MATHEMATICAL PASTIMES

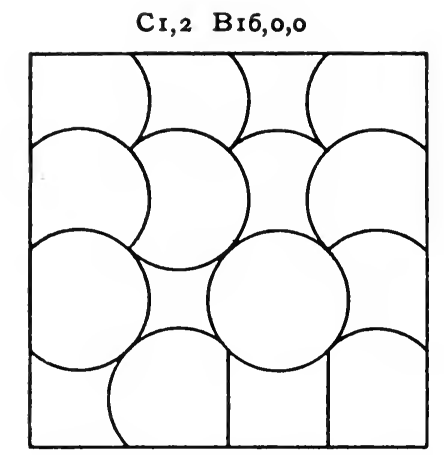<smiles>C1CCCCCCCCCCCCCCCC1</smiles><smiles>C1CCCCCCCCCCCCCCCCCCCCCCCCCCCCCCCC1</smiles><smiles>C1CCCCCCCCCCCCCCCCCCCCCCCCCCCCCCCCCCCC1</smiles>

Fig. 89 .

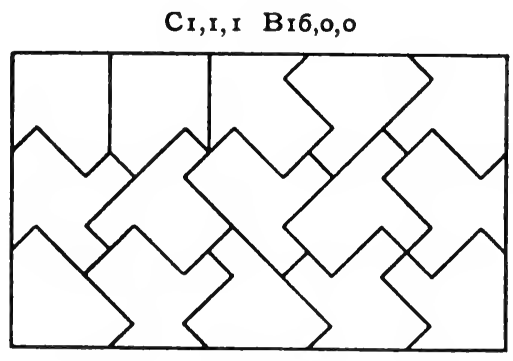

Fig. 90. 
THE TRANSFORMATION OF PARTI
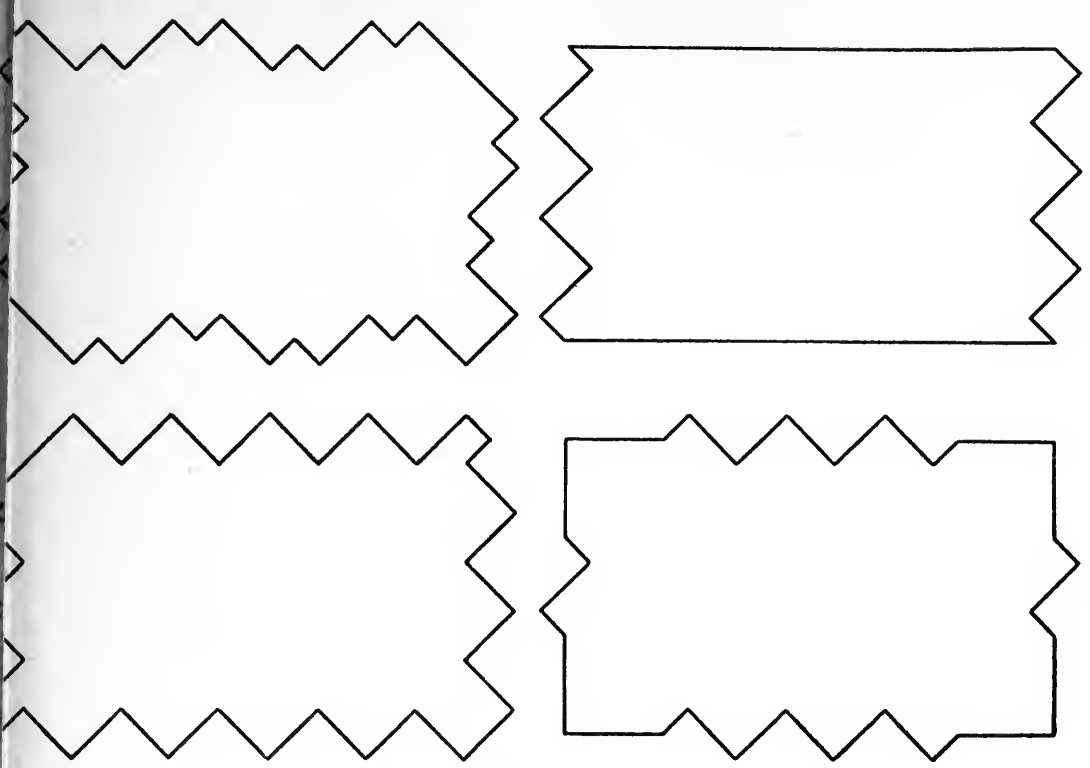

Fig. 9I.
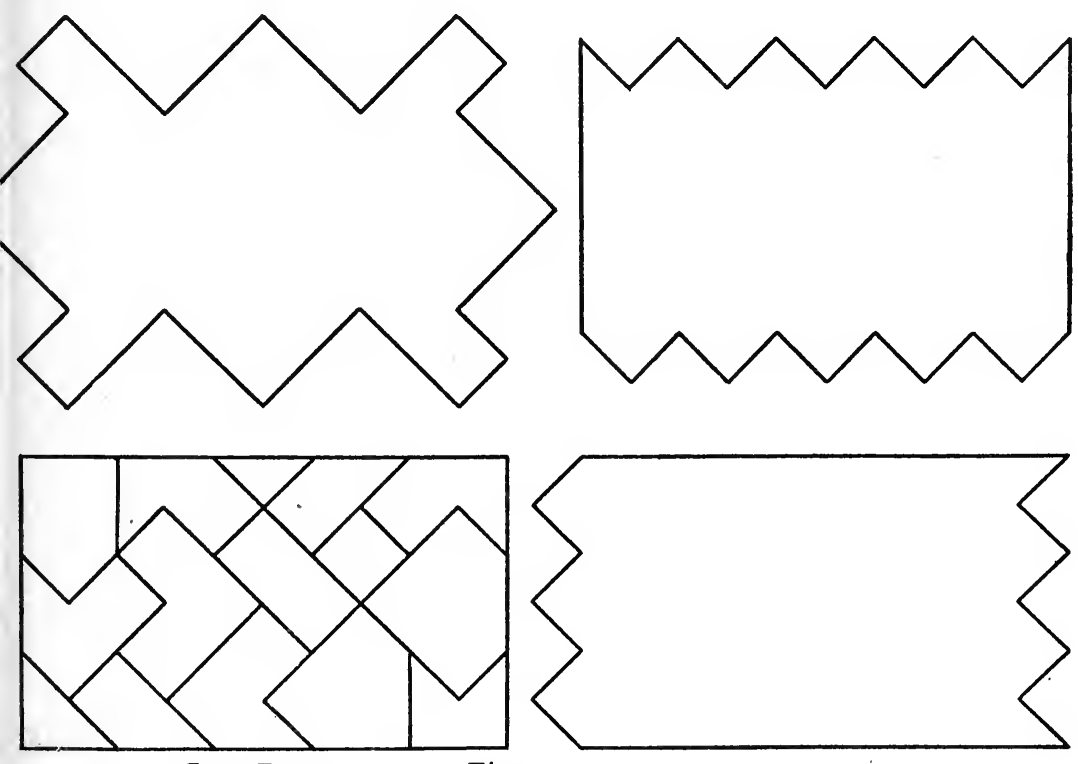

$\mathrm{CI}_{1,2} \mathrm{BI} 6,0,0$

Fig. 92. 

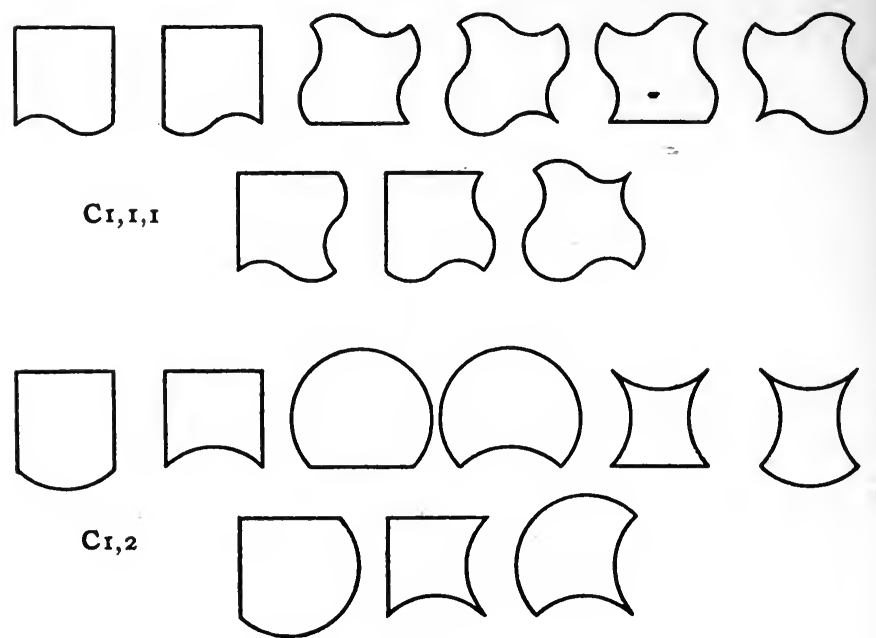

Fig. 93.

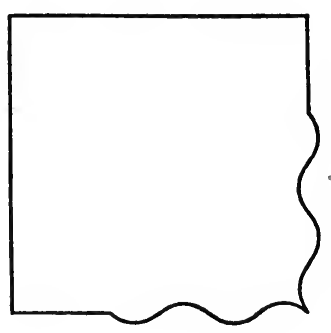

Fig. 94 .

$C_{1, I, I} \quad B 8,2,2$
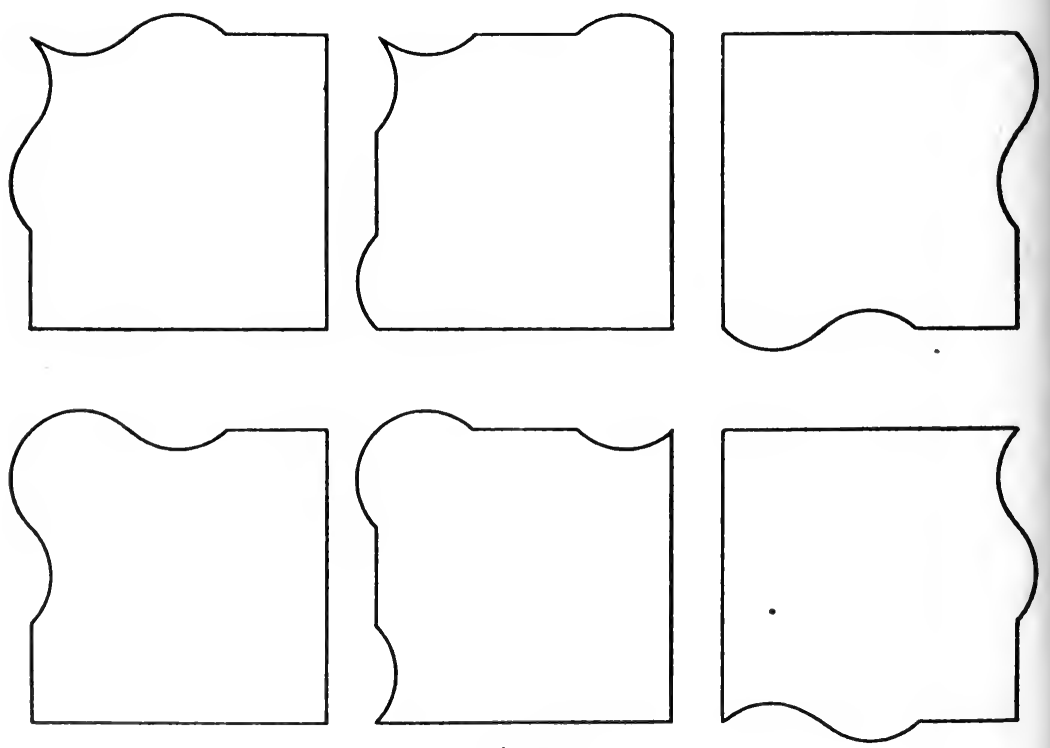

Fig. 95. 
58. In fig. $96 a$ the set of 24 squares involving five colours unrepeated, are shewn assembled for the contact system I to I, 2 to 3,4 to 5 and a transformation according to fig. $96 b$ is shewn in fig. $96 c$. The symmetry about the central horizontal line is to be remarked.

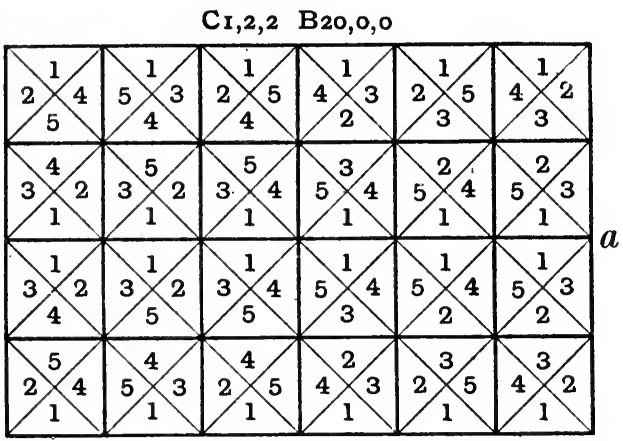

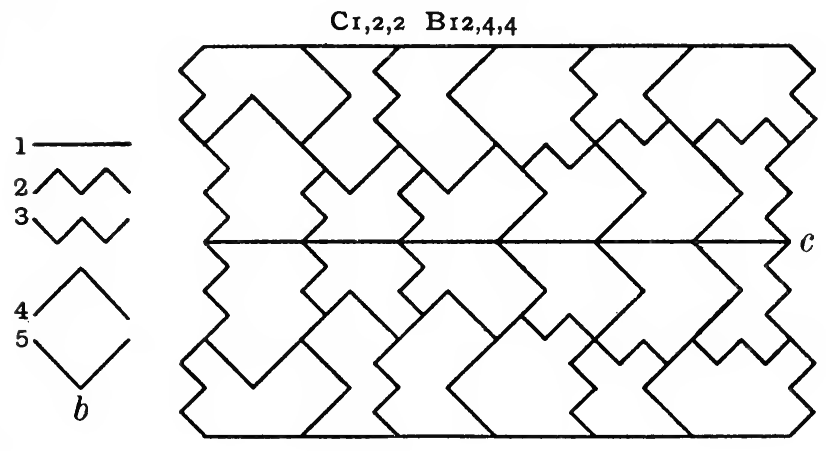

Fig. 96.

It is obvious that there is ample room for experiments with this interesting set. 


\section{PART III}

\section{'THE DESIGN OF 'REPEATING PATTERNS' FOR DECORATIVE WORK}

The story without an end that angels throng to hear.

M. F. TUPPER.

59. The ideas which have been prominent in Parts I and II lead to a most interesting Pastime-the design of repeating patterns for various kinds of decoration. In Part I the notion was to connect colours with the compartments of various polygons, usually triangles and squares, in such wise as to realise every possible combination of colours on the compartments. The set thus formed has the property that no two pieces are alike. There are no duplicates. Smaller sets are chosen from these according to definite principles and laws but the pieces are in every case differently coloured. In the transformations of Part II the passage is made from pieces of the same shape but differently coloured to pieces of the same colour but differently shaped. The transformations do not affect the cardinal property that no two pieces of any set are alike.

It was shewn that the pieces of a set can be assembled so as to fit inside a boundary which can be specified as to colour or shape and that, for a given set of pieces, the different boundaries that can be predicted exist, for various contact systems, in very large numbers.

We now examine what can be done with pieces which, far from being all different, are all exactly similar in shape and size.

Certain pieces of the same size and shape can, everybody knows, be. fitted together so as to completely cover any floor, wall, ceiling or other flat surface, and no attention need be paid to the boundary. The pieces, when assembled, can be cut along any desired boundary. For practical purposes the boundary may be ignored.

The simplest repeating patterns are shewn in fig. 97 and are met with everywhere. 
We make a systematic search for pieces of other shapes which possess the same property of completely covering any flat surface by simple repetition.

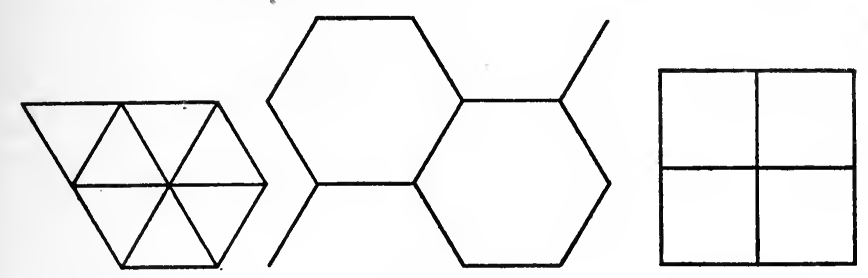

Fig. 97.

These 'repeating patterns' are everywhere visible in considerable variety.

Public buildings and private homes exhibit examples on floors, walls and ceilings. Street pavements, palings, furniture, wearing apparel, woven fabrics and artistic designs of all sorts bring them constantly before our eyes.

We feel that the ideas brought before the reader in this book will enable him to take quite a fresh interest in these matters.

Without making any pretence of exhausting the subject we base our study upon the equilateral triangle, the square, and the regular hexagon. These are probably the most important bases.

I saw it budding in beauty; I felt the magic of its smile.

M. F. TUPPER.

60. It is a very trivial fact that the equilateral triangle is a repeating pattern for any flat surface; but one useful remark may be made, viz. that in such an assemblage, a portion of which is represented above, the triangle has always just two aspects or orientations, because the fitting is invariably so that of two adjacent pieces one is always like the other, only upside down, as may be readily seen.

This is not the case in either the square or hexagonal pavements, which present only one aspect.

The equilateral triangle, which as a repeating pattern has two aspects, gives immediate rise to other repeating patterns upon very simple principles.

If we can in any manner separate it into three or more parts of the same size and shape we obtain again a repeating pattern.

M. P. 
For example we can as in fig. 98 from the centre $O$ draw perpendiculars upon the sides and obtain the equal and similar quadrilaterals of which one is $O D B F$, a repeating pattern which in an assemblage has $3 \times 2$ or 6 aspects.

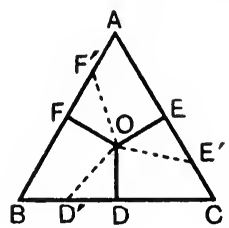

Fig. 98.

Also we may draw the lines $O D^{\prime}, O E^{\prime}, O F^{\prime}$ making equal angles with $O D, O E, O F$ and obtain the repeating pattern $O D^{\prime} B F^{\prime}$.

This system of quadrilaterals has two opposite angles $60^{\circ}$ and $120^{\circ}$ respectively.

61. Similarly from the square we can at once derive an unlimited number of repeating patterns by drawing lines, straight or not, through the centre as in fig. 99.
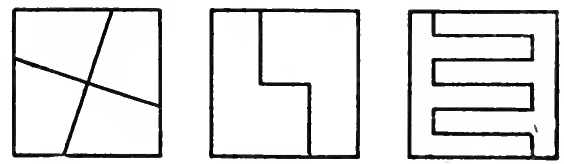

Fig. 99 .

The first of these figures shews a separation into two or four parts, the second into two parts, the third into two parts. There is no limit to the number of such separations.

Any number of squares may be placed in contact to form a pattern, and a little consideration shews that it follows that every rectangle is a repeating pattern.

Who made the spider parallels design,

Sure as de Moivre, without rule or line?

Essay on Man.

If we draw a series of equidistant parallel lines and cut them with any other series of equidistant parallels, we see at once that every parallelogram is a repeating pattern with one aspect, and since every parallelogram can be separated into two equal and similar triangles; and vice versa every triangle is the half of a 
parallelogram; it follows that every triangle is a repeating pattern, with two aspects.

As a general principle, if any repeating pattern can be divided into two or more equal and similar parts, a part thus formed is a repeating pattern*.

62. When we assemble a number of patterns so as to cover a flat space we can always observe combinations of patterns which are themselves patterns. If the pattern has a certain number of aspects in the assemblage and we draw a boundary which includes one pattern of each aspect, the figure enclosed by the boundary is a pattern with one aspect.

For example the equilateral triangle has two aspects, and we can draw a regular hexagon which encloses six triangles, three of each aspect. Thence we conclude at once that the regular hexagon is a pattern having one aspect.

63. The regular hexagon may be derived as a repeating pattern from the equilateral triangle in another interesting manner.

We can colour an assemblage of triangles as in fig. IOO $a$ with two colours so that each triangle is adjacent to three triangles of a different colour.
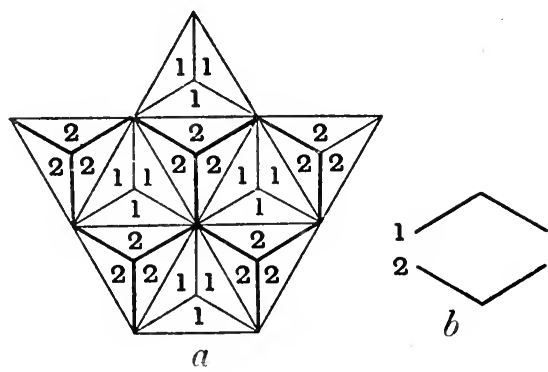

Fig. I00.

It is by others termed a fesse between two gemels. And that is as farr from the marke as the other; for a gemel ever goeth by paires, or couples, and not to be separated.

R. Holme, Academy of Armory, i. iii. 77.

We can now transform from colour to shape by the method of Part II on the contact system I to 2, taking for the colours

* Triangles may be taken to be equal and similar in plane geometry when they can be made to exactly coincide by movements in the plane; a movement such as 'turning over' is not in view. 
the boundaries as in fig. $100 b$, when we find that the triangles coloured 2 entirely disappear and the triangles coloured I become regular hexagons.

We have transformed the tessellation from being triangular to being hexagonal.

64. The mathematical reader will recall that, in space, the cube and the rhombic dodecahedron are similarly associated. In that case we start with a number of cubes and give them two colours as in a three-dimensional chessboard. The cubes coloured 2 are divided into six portions by lines joining the centre to the eight summits; each portion being a pyramid whose base is a face of the cube and whose height is equal to half of the cube's edge. Each cube coloured I is adjacent to six cubes coloured 2, and transformation upon the contact system I to 2 with pyramidal boundaries causes the cubes coloured 2 to disappear and those coloured I to become rhombic dodecahedra.

Space is thus exhibited as partitioned into rhombic dodecahedra, a fact of great importance in crystallography and other parts of applied mathematics and mathematical physics.

The foregoing considerations bring out the importance of designing patterns which possess symmetry about one or more axes; from these other patterns can be at once derived.

65. In Part II we adopted certain forms of compartment boundaries in association with the contact systems, and the design of such boundaries was subject to the transformed pieces being readily handled. This operated as a considerable restriction which can now be removed because our pieces are merely delineated; they are not handled at all. The restriction took two forms.
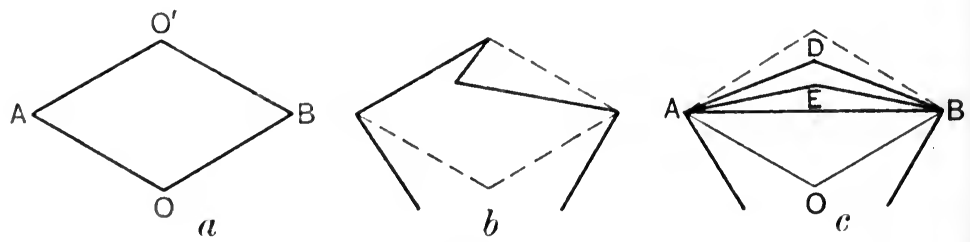

Fig. 101.

$A O^{\prime} B O$ in fig. IOI $a$ being the compartment parallelogram, in clrawing the new boundary between $A$ and $B$ and within the 
parallelogram we decided that no perpendicular to $A B$ should cut the new boundary in more than one point; because if it did so the piece would be inconvenient to handle. Again it was tacitly agreed that the portion of the piece bounded by the new boundary line and $A O B$ should have no holes in it. Both of these restrictions can now be removed and we can deal with shapes such as in fig. Ior $b$ and Ior $c$ where in the second case there is an inner as well as an outer boundary, the triangular piece $A E B$ being cut out.

\section{THE TRIANGLE BASE}

66. Consider the triangle to be divided into compartments numbered I, 2, 3 so that, travelling clockwise round the figure, the numbers are in ascending order of magnitude as in fig. IO2 $a$.
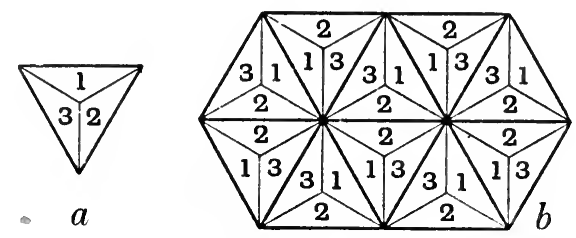

Fig. I02.

It is not quite a trivial remark that any number of triangles identical with this one can be assembled so that the compartments numbered I, 2, 3 are adjacent to others numbered I, 2, 3 respectively.

The annexed diagram, fig. I02 $b$, establishes this and shews that the number of aspects of the triangle is not increased by the numbering. We knew before that it could not be less than two. We know now that it remains precisely two.

Also the internal structure is always the same.

The reader who has become acquainted with Part II will observe that the straight line boundaries of the compartments of the triangles can be altered in many ways so that the pieces remain of the same shape and size. In other words the numbered triangular piece can have its shape altered to an indefinite extent and still preserve the property of being a repeating pattern.

The assemblage under examination has the contact system I to $\mathrm{I}, 2$ to 2,3 to 3 , and we may employ any of the boundaries 
which belong to the system. The numbers $1,2,3$ need not differ from one another.

The particular cases I, I, I; I, I, 2 are included in the discussion.

67. We are about to study the design of repeating patterns and the reader will readily realise that a pretty pattern must always be an object, and a principal object, in the designer's mind. This can best be accomplished by paying attention to symmetry of shape.

We must learn how to select boundaries which will produce symmetrical patterns. This matter must be studied in the case of each contact system and each base.

To assist us we must have a typical boundary before us, say $A$ in fig. $103 a$, and associate with it another boundary which we will call the inverse of $A$ and denote by $i A$.

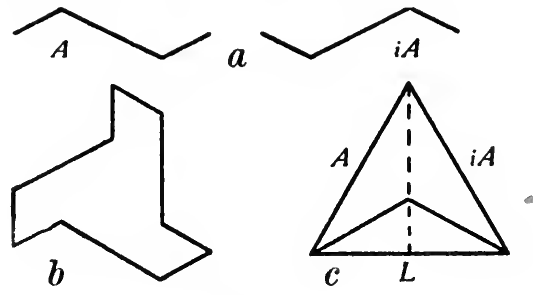

Fig. 103.

$i A$ is the reflexion of $A$ in a mirror placed to its right, or it is $A$ rotated about its right-hand extremity, in a plane perpendicular to the plane of the paper, until it occupies the position of $i A$.

It will be noticed that if $A$ be the original single straight line, inversion does not alter it.

In the first place it is clear that if the numbers I, 2, 3 are associated with the same boundary $A$, however we choose $A$, the piece will possess trilateral symmetry.

For example, for the typical boundary we get fig. $103 b$.

The only other kind of symmetry is that about an axis which bisects one of the angles of the triangle; say about the line chain-dotted in fig. $103 c$.

As regards the sides which meet at the angle fixed upon, the only possibility is to associate them with the boundaries $A$, iA respectively. 
The reader is reminded that a compartment-boundary is to be viewed from the centre of the base, looking outwards.

In regard to the third side it may remain associated with the single straight line.

We call this $L$ for short.

Notice also that the shape is altered when we interchange $A$ and $i A$.

For the typical boundary we thus get the two patterns of fig. IO4 $a$ and $b$.
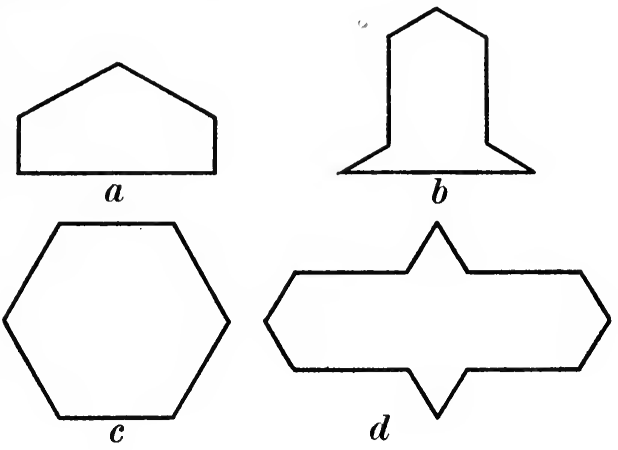

Fig. IO4.

Inspection of the numbered diagrams shews that we can pass from them to repeating patterns, fig. IO $c c$ and $d$, of which the first is the regular hexagon again. In both cases the sides $L$ have been made adjacent. Other patterns are obtained by making the sides $A$ or the sides $i A$ adjacent in each case.

68. The particular case 2 equal to 3 may be noticed. It is interesting from the circumstance that all pieces of this type may be assembled so as to have either two or six aspects. This is made clear by the diagrams in fig. IO5 in which the diagram on the left exhibits two aspects and that on the right six.

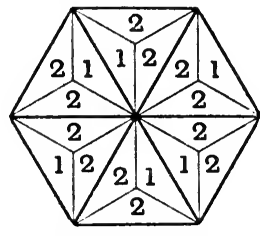

Two aspects

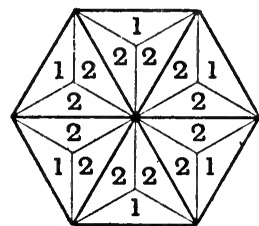

Six aspects

Fig. 105. 
In fig. 106 some patterns and assemblages belonging to this system are given and in particular the 'Helmet' pattern is
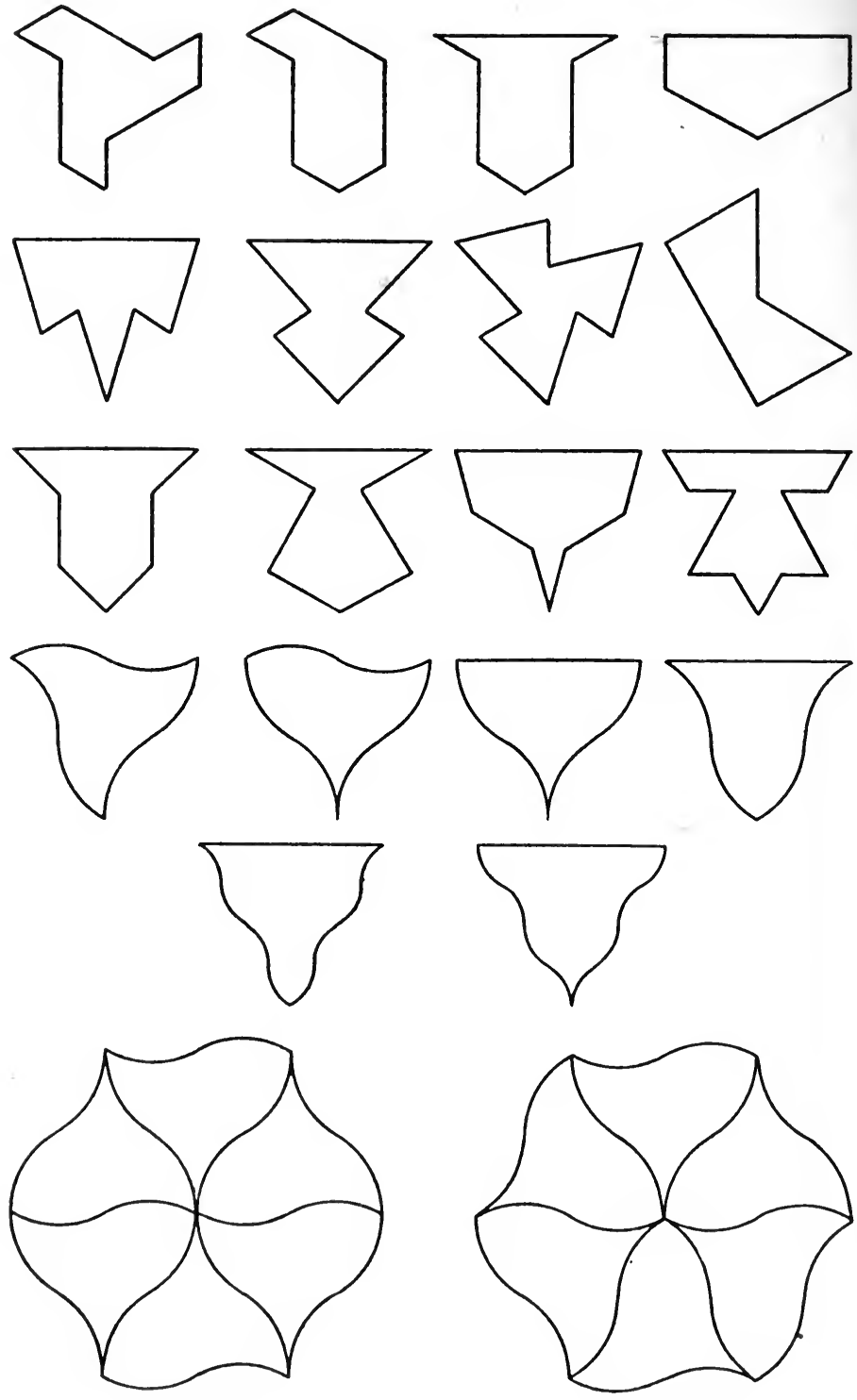

Two aspects

Six aspects

Fig. 106. 
shewn assembled so as to exhibit two aspects, on the left, and six aspects, on the right.

Besides the equilateral triangle, since every triangle is a repeating pattern, we may take as base the isosceles triangle in general as in fig. IO7 and the isosceles right-angled triangle in particular.

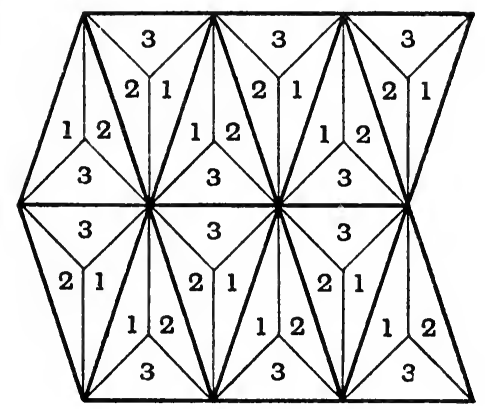

Fig. 107.

The diagram shews that, for this contact system, the treatment is the same. The reader will find no difficulty with this general case.

So that the art and practic part of life

Must be the mistress to this theoric.

Hen. $V$. I. i.

69. A second and entirely different system of patterns is obtainable from the contact system I to I, 2 to 3 which we call $C_{\mathrm{I}, 2}$.

Inspection of the depicted assemblages of six triangles in the form of a hexagon as shewn in fig. 108 shews that the piece has six aspects in each of two constructions, which are at the disposition of the designer.

Part II again shews us how to alter the shape of the piece so that it will remain a repeating pattern.

Herm. Methinks I see these things with parted eye, When ev'rything seems double.

$\mathrm{Hel}$. So methinks :

And I have found Demetrius like a gemel, Mine own, and not mine own.

Mids. N. Dr. IV. i.

The boundary of compartment $\mathrm{I}$ is altered in any manner which appertains to the first system already dealt ivith, whilst 

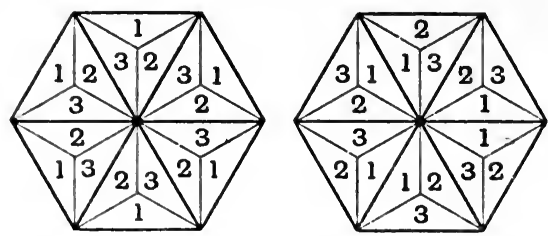

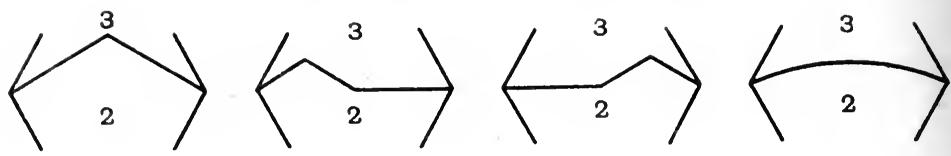
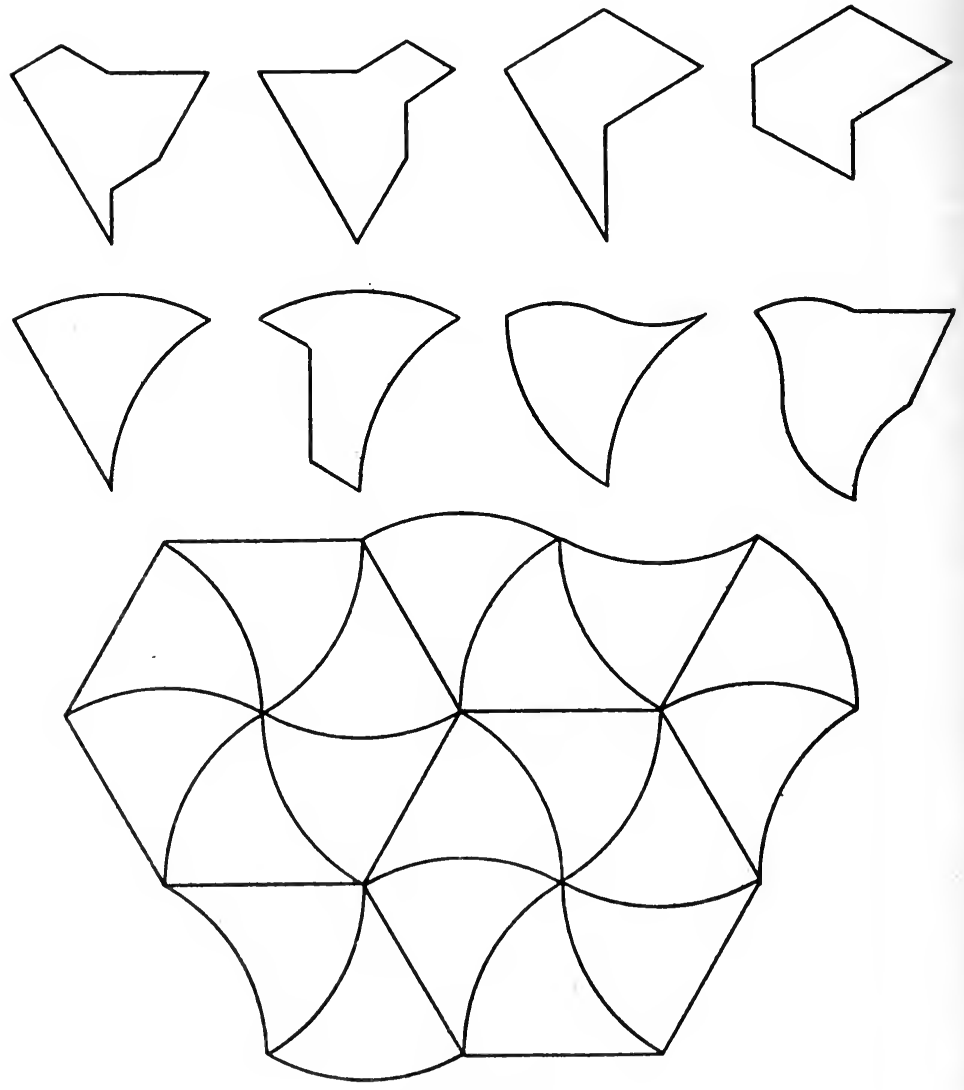

Six aspects

Fig. 108. 
for compartments 2 and 3 we have the boundaries of Part II as in the numbered row of fig. 108 where the 3 rd is the inverse of the 2 nd, while the Ist and $4^{\text {th }}$ are self-inverse.

Some examples of repeating patterns, issuing from this system, and an assemblage are given in the same fig. (IO8).

Symmetry can be secured by taking six identical pieces as in the annexed diagram, fig. $109 a$, which is a well-known pattern composed of three hexagons.
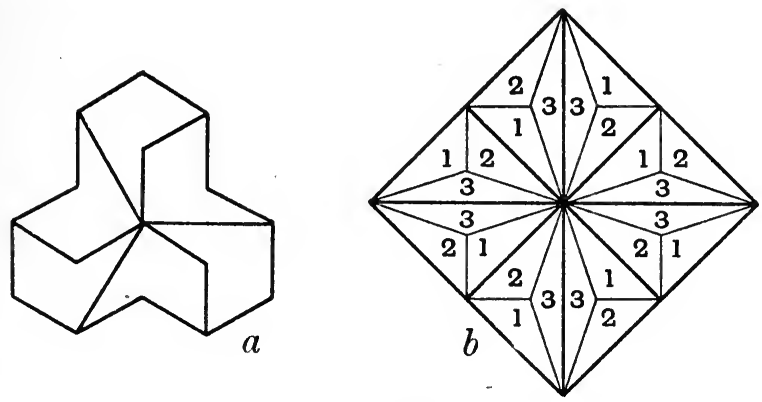

Fig. I09.

The isosceles right-angled triangle is also available for this contact system, as shewn by the diagram of fig. $109 b$. The system is I to 2,3 to 3 , and the derived patterns have four aspects when assembled.

\section{THE SQUARE BASE}

For the wals glistered with red marble and pargeting of divers colours, yea all the house was paved with checker and tesseled worke.

KNOLLES' Hist. of Turks.

But I of these will wrest an alphabet,

And by still practice learn to know thy meaning.

Tit. Andron. 111. ii.

70. We recall that the piece does not, of necessity, have more than one aspect.

Numbering the compartments as in fig. I Io $a$ we can always assemble according to the contact system I to I, 2 to 2,3 to 3 , 4 to 4 which is denoted by $C_{\mathrm{I}, \mathrm{I}, \mathrm{I}, \mathrm{I} \text {. }}$

This is shewn in fig. I Io $b$, a diagram which can be repeated indefinitely. 
The arrangement proves that it is possible in one way only and that the piece has two aspects, one being the other rotated through two right angles.

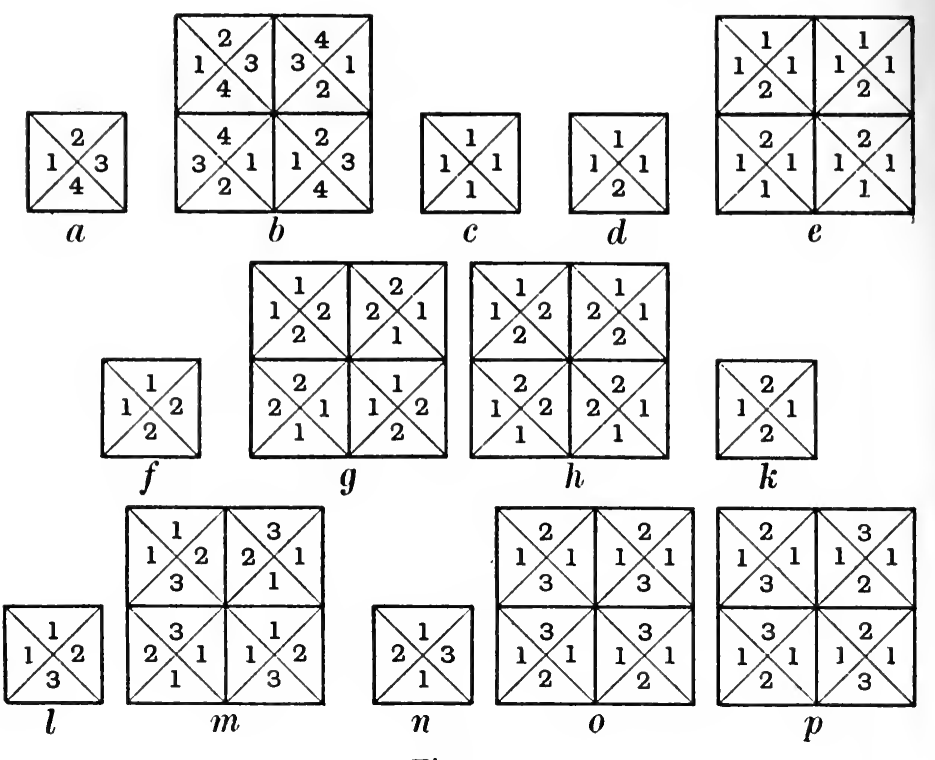

Fig. I 10.

In the particular cases: fig. I Io $c$ has one arrangement with one aspect of piece.

Fig. I IO $d$ has only one symmetrical arrangement which deserves consideration as shewn in fig. I IO $e$ with two aspects of piece.

The piece in fig. I Iof has two arrangements shewn in fig. I IO $g$ (two aspects), in fig. I Ioh (four aspects).

The piece in fig. I Io $k$ has obviously one arrangement with one aspect of piece.

The piece in fig. I Io $l$ has one arrangement with two aspects as shewn in fig. IIO $m$; and finally the piece in fig. I IO $n$ has the arrangements of fig. IIO, $o$ and $p$, each with two aspects.

71. To construct repeating patterns we take four shapes of boundary drawn from the first system of boundaries, taking care to choose those that are appropriate to the square base. 
We are restricted to the square $A O B O^{\prime}$ in fig. II I and no circular arc employed must lie outside of it at any portion of its course between $A$ and $B$. When the arc passes through $A$ the centre from which it is struck should be taken upon either $A O^{\prime}$
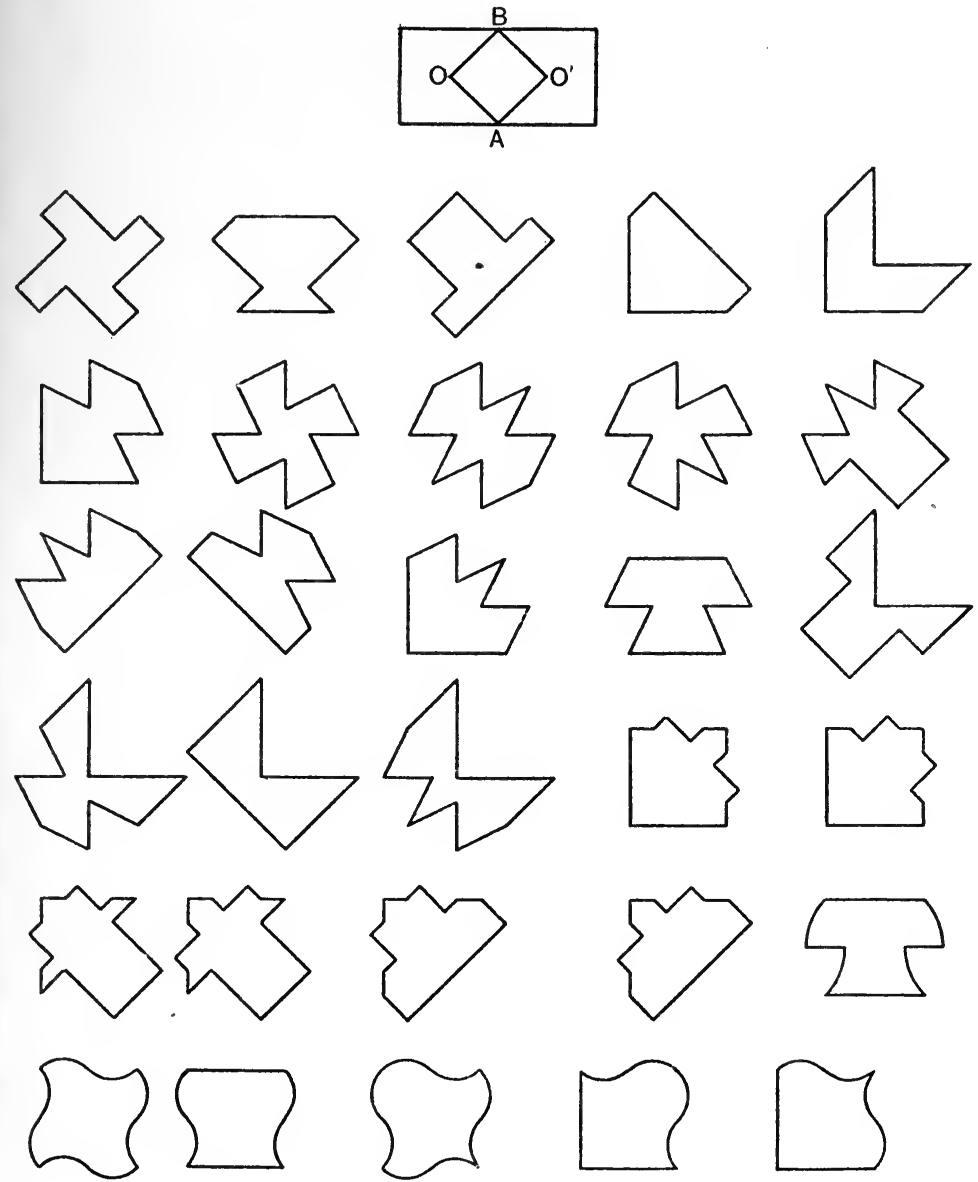

Fig. III.

or $A O$ produced if necessary so that it touches $O A$ or $O^{\prime} A$ at the point $A$. Part II points out convenient forms and leads us to a variety of patterns of which samples are shewn in fig. I I I and assemblages in fig. I 12 where $a$ and $b$ exhibit two aspects and $c$ four. 
Seizes the prompt occasion, makes the thought

Start into instant action, and at once

Plans and performs, resolves and executes!

HANNAH MORE.
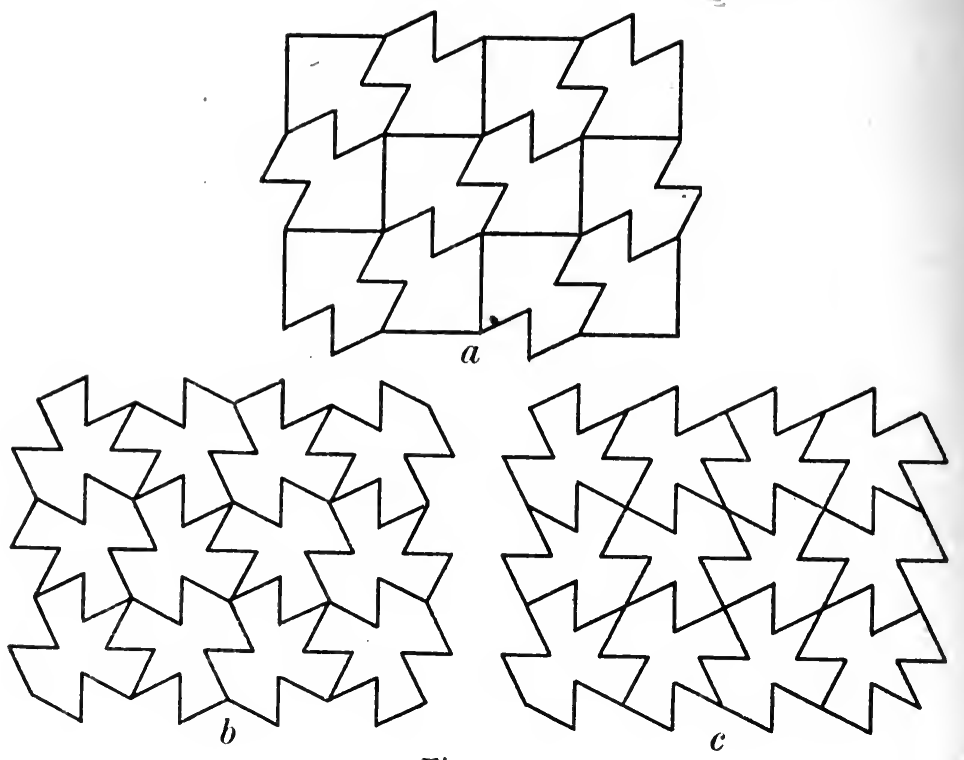

Fig. 112.

Two other assemblages are shewn in fig. I 3 where fig. $a$ exhibits two aspects and $b$ four.

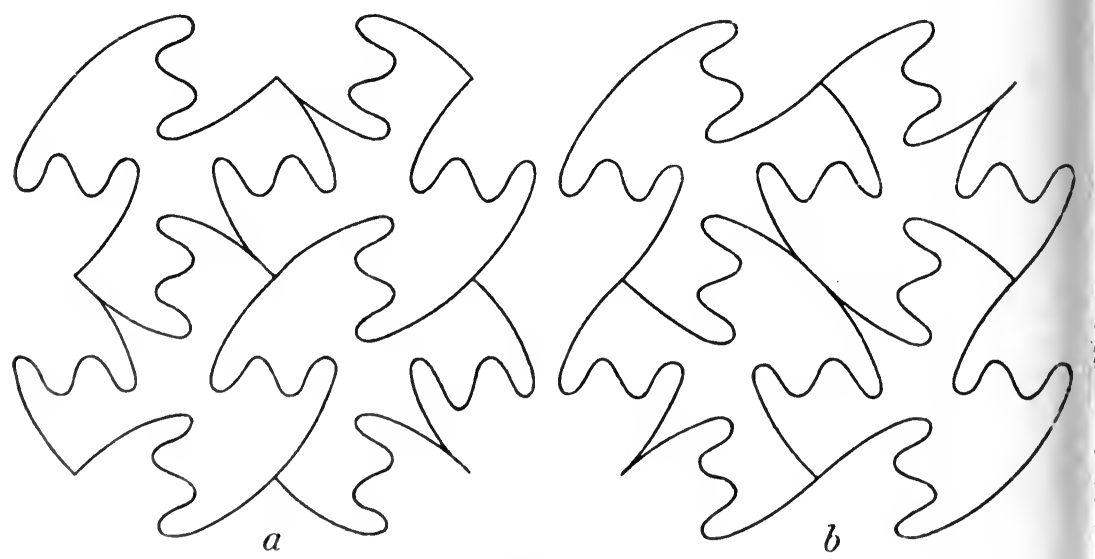

Fig. I 13 . 


\section{SYMMETRY OF PATTERN}

Veluti in speculum.

Let no face be kept in mind, But the fair of Rosalind.

As You Like It, III. ii.

72. We now consider some more principles which lead to symmetry of pattern.

We take four compartment boundaries as in fig. I $4 a$; we have already defined $i A$; we call the one beneath $i A$ the complement of $A$ and denote it by $c A$. It is called the 'complement' because the compartment with the boundary $c A$ will fit into (lie adjacent to) the compartment with the boundary $A$.

So also the boundary beneath $A$ is called the complement of the inverse of $A$.

It will be noticed that $c i A$ and $c A$ are reflexions of $A$ and $i A$ in the horizontal chain-line delineated.

Also that $i c A$ is the same as $c i A$ and that $c c A, i i A$ both leave $A$ unaltered.
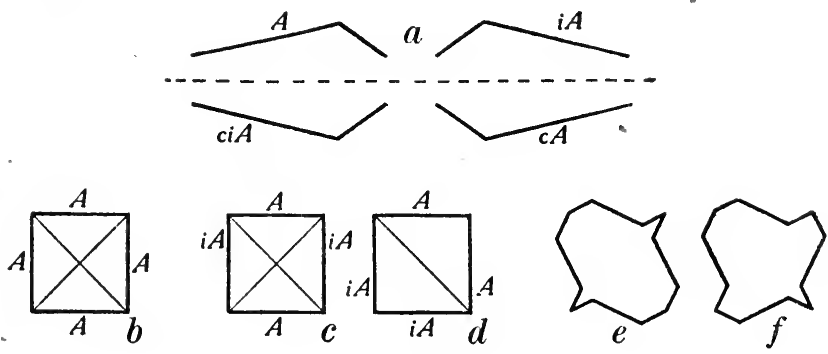

Fig. I I 4 .

$c A$ and $A$ on the one hand and $c i A$ and $i A$ on the other have to do with the second kind of contact between compartments.

We are immediately concerned only with the first kind of contact and we see that we may have, as in fig. I $44 b$, the same boundary to each compartment giving quadrilateral symmetry.

Or we may have the designs with diagonal symmetry in fig. II $4 c$ and $d$ where the first has symmetry about both diagonals, because both bisect angles contained by $A$ and $i A$; and the second has only symmetry about the diagonal drawn because the other does not satisfy the $A$ and $i A$ condition.

Examples are shewn in fig. I $4 e$ and $f$. 
We will have, if this fadge not, an antick. I beseech you, follow.

Love's L. L. v. i.

73. To obtain symmetry about a straight line through the centre parallel to a side the form that must be taken is that in fig. I I $5 a$ of which an example is fig. I $5 b$.
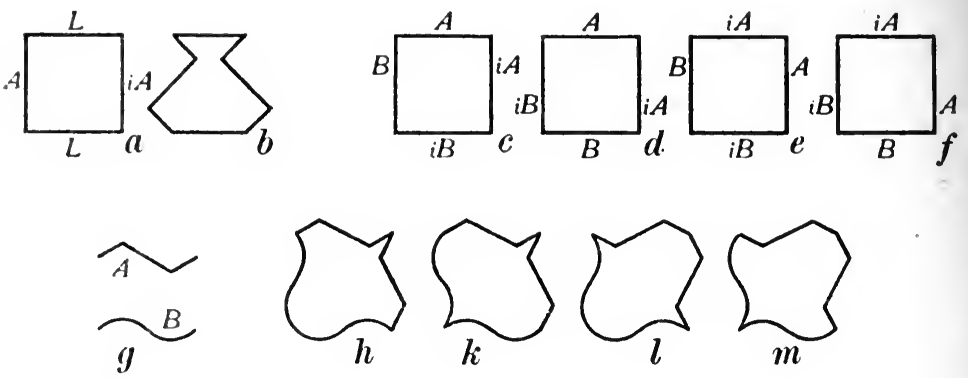

Fig. 115 .

With two boundary forms $A, B$ we have four forms, fig. I I $5 c, d, e, f$, each of which has symmetry about one diagonal.

Examples for the boundaries in fig. I $5 g$ are shewn in fig. I $5 h, k, l$, and $m$.

74. The next contact system to study is that in which we have $I$ to $I, 3$ to 3,2 to 4 and inspection of the diagram in fig. I 6 shews that there is one arrangement with two aspects of piece.

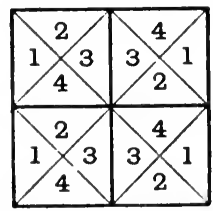

Fig. $11 \%$.

This is also the case when $I$ and 2 are identical.

For the boundaries of compartments 2 and 4 we choose from those brought forward in Part II that are appropriate to the square base.

Patterns of quite a new character emerge, as is evident from the samples in fig. 117. 

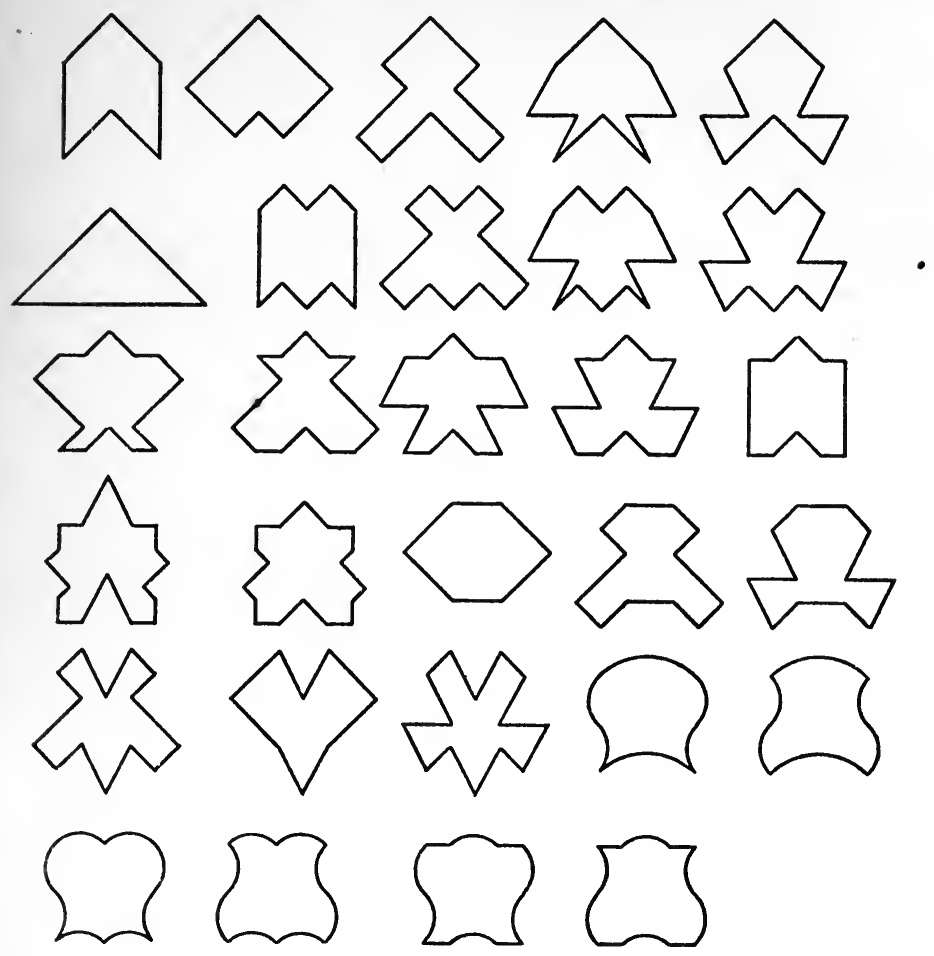

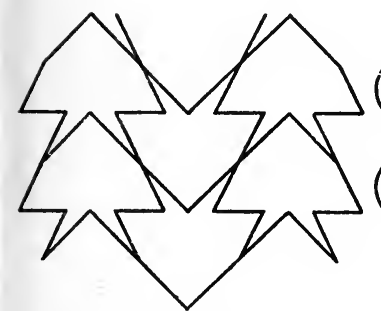

Two aspects

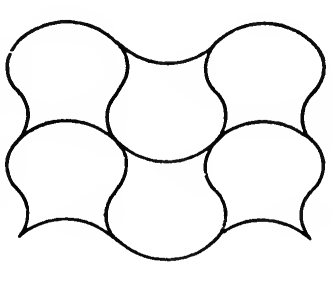

Two aspects

Fig. 117.

75. Here the only possible symmetry is that about an axis through the centre parallel to a side. The general forms are as in fig. I $8 a$ and $b$, where $B, A$ belong to the first and second kinds of contact respectively.

In particular either $A$ or $B$ may have the form $L$.

M. P. 

$A=i A$.

$A$ must also be symmetrical about its extremities, so that

As an example, for the forms in fig. I $8 c$ we find fig. I I $8 d$, a piece with windows.
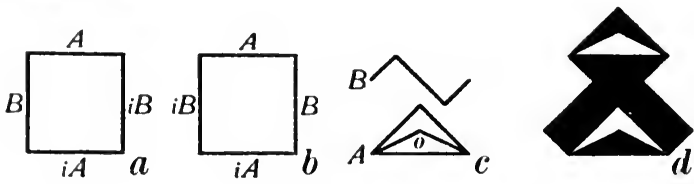

Fig. I 18 .

(Deh come è ver che) subito travato

Il bello piace a chi non è malato.

BRACCIOLINI.

76. A few minutes trial will convince the reader that the contact system I to I, 2 to 2,3 to 4 is impossible unless I and 2 are identical.

For the case specified we have the arrangement shewn in the diagram on the left of fig. I I 9 involving four aspects, and no other essentially different from it.
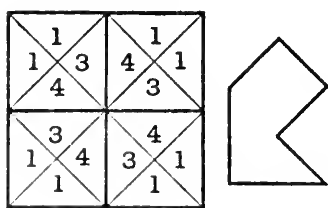

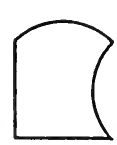

Fig. I 19.
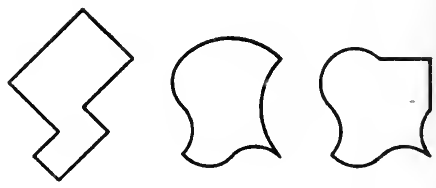

This system does not lend itsclf to interesting symmetrical patterns, for two reasons. In the first place 3 and 4 are not opposite compartments, and in the second the inverse principle cannot be applied to the two identical compartments I.

However, a few forms are given in fig. I I9.

77. A more interesting set of patterns arises from the contact system I to 3, 2 to 4. Here the contacts are of oppositely situated compartments and the inverse principle is also available.

The arrangement is shewn in fig. $120 a$. It involves only one aspect of piece.

It can be repeated to any extent on all sides.

Exceptionally the piece in fig. $120 b$ with the contact system I to 3 , can be assembled in three different ways having one, two and four aspects respectively. 
This is shewn by the diagrams $120 e$ with one aspect, $120 d$ with two aspects and $120 e$ with four aspects.

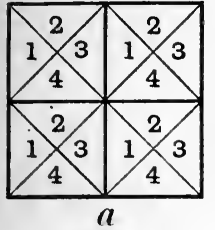

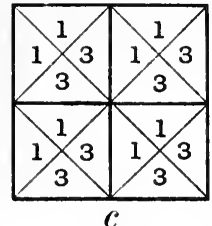

Fig. I20.
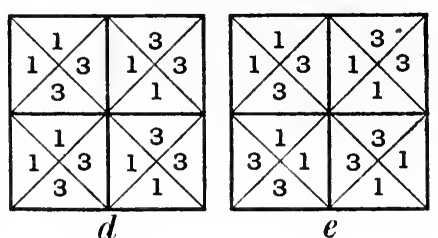

Some patterns and assemblages are shewn in fig. I 2 I where $a$ has one aspect, $b$ two aspects, $c$ four aspects and $d$ one aspect.
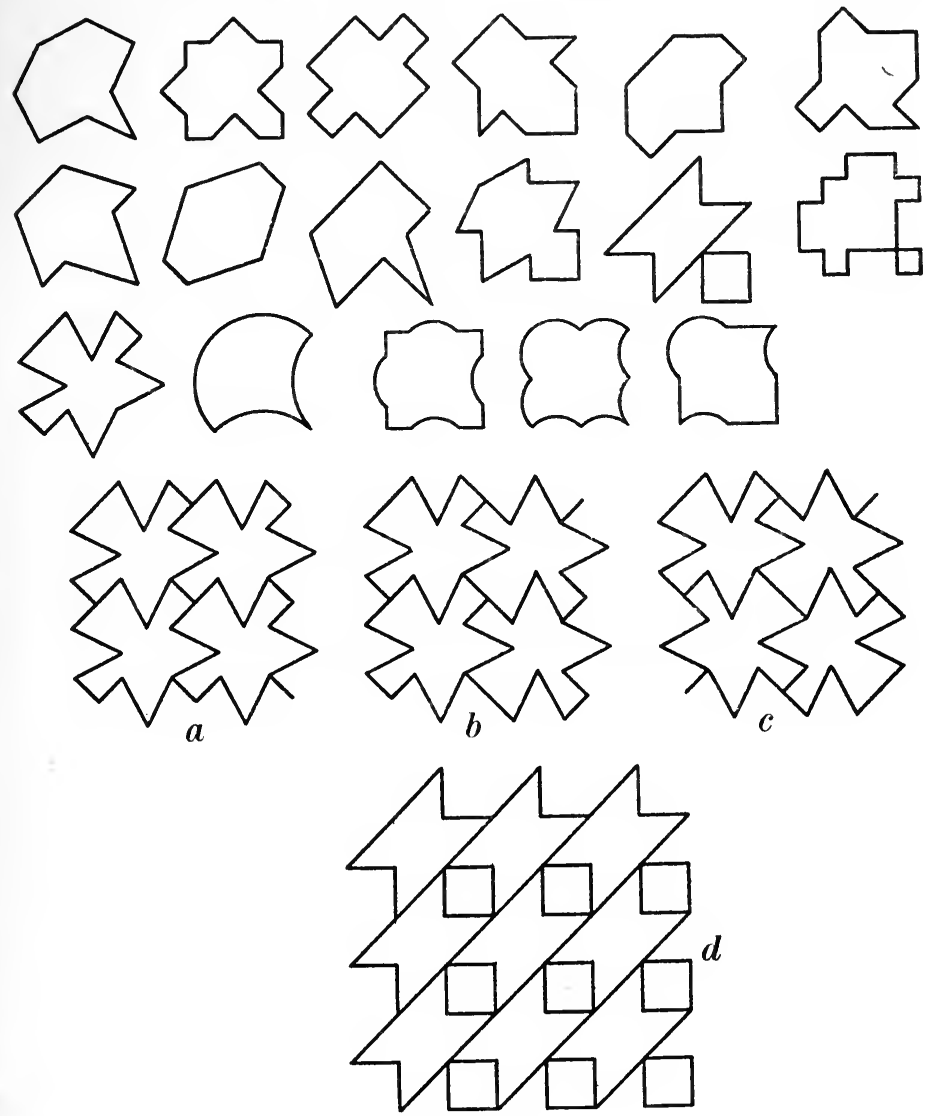

Fig. I2I. 
78. The three symmetrical forms are shewn in figs. $122 a, b$, and $c$ where for the first of these $A$ must be identical with its inverse.

Examples of the last two are figs. $122 d$ and $e$, and of the first fig. $122 f$.
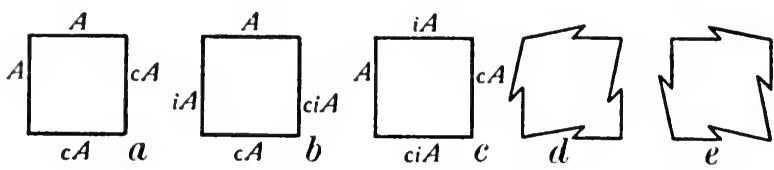

Fig. 122 .

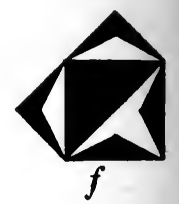

79. Lastly we have the contact system 1 to 4,2 to 3 .

The arrangement is as in fig. 123.

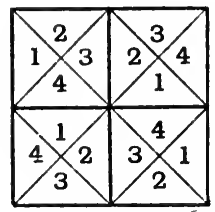

Four aspects

Fig. I 23.

The arrangement can be, clearly, repeated to any extent.

Some examples of the patterns that arise are given in fig. 124 where assemblage $a$ has two aspects and assemblage $b$ has four.

The reader will observe that the tessellation (fig. I $24 b$ ) is composed of two sets of hexagons at right angles to one another.

Each hexagon contains four equilateral pentagons, one of each aspect.

80. For symmetry about an axis parallel to a side we have fig. $125 a$ where $A$ is a form which is identical with its inverse; and for symmetry about a diagonal the two forms in fig. $125 b$ and $c$ when $A$ is as in fig. $125 d$, the triangle numbered zero being cut out; the pattern arising from the last form but one is curious, as shewn in fig. $125 e$.

It has four aspects and requires four colours when assembled.

In the assemblage shewn in fig. $125 f$ the numbers denote different colours.

Pieces having the same aspect are given the same colour. 
Mrams

$0 \Omega 5 \sqrt{3}$

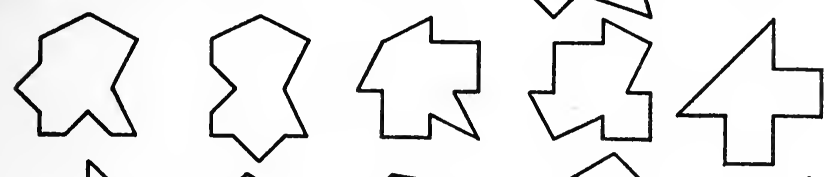

品 $\square$

Gूलूहु

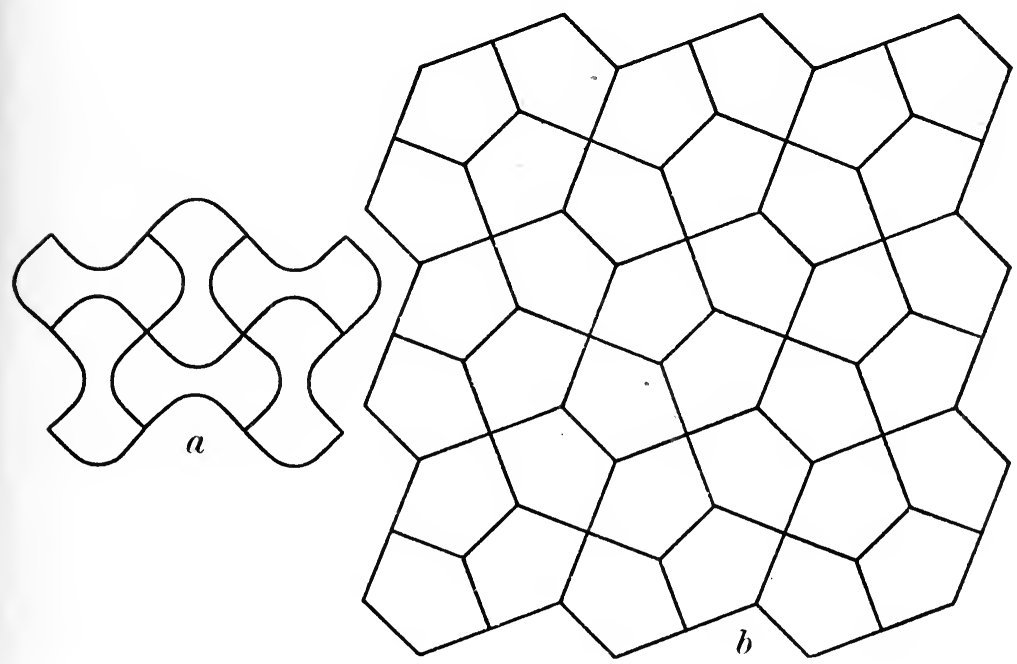

Fig. I24. 

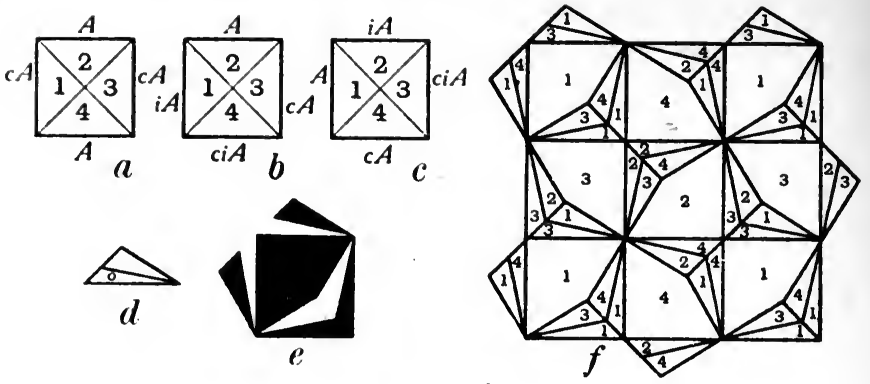

Fig. 125 .

Parfois dans un coin triste et noir pousse une fleur.

FRANÇOIS COPPÉE.

See'st thou the gaze-hound! how with glance severe From the close herd he marks the destin'd deer.

STEELE'S Miscellanie.

81. The tessellation of pentagons that has been depicted is one of the most remarkable that can be met with in the subject.

If the patterns that have been given above, for the contact system under examination 1 to 4,2 to 3 , be inspected they will be found to include three convex pentagons, out of an infinite number that it is possible to construct. In particular the first given of the three is equilateral.

Taking the base square $A B C D$ as in fig. I 26 we have to find compartment boundaries for the contacts I to 4,2 to 3 that will

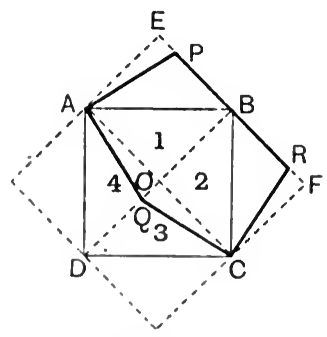

Fig. 126.

lie wholly inside the compartment square $A O B E$ and the three others similarly situated in regard to the other sides $B C, C D, D A$. In order that the resulting pattern may be a convex pentagon we choose any point $P$ in $E B$ and take a compartment boundary 
$A P B$ for the compartment $\mathrm{I}$. This necessitates the choice of a similarly situated point $Q$ on the line $O D$ and the compartment boundary $A Q D$ for the compartment 4 .

Thus the projection $A P B$ on one pattern will fit into the indent $A Q D$ in another.

We now select for the compartment 3, the boundary $D Q C$ (an indent). This selection necessitates the projecting boundary $B R C, B R C$ being similar to $D Q C$ and the inverse of $A P B$, for the compartment 2 .

The result is the convex repeating pentagonal pattern APRCQ.

It will be noticed that $P A Q$ and $R C Q$ are necessarily both right angles.

We can thus obtain an infinite number of convex pentagons as repeating patterns, by simply varying the situation of $P$ upon the line $E B$.

If $P$ coincides with $B$ the pentagon degenerates to the square $A B C D$.

If $P$ coincides with $E$ the pentagon degenerates to the rectangle $A E F C$.

In order to make the pentagon equilateral we first notice that by construction it has four sides equal, viz. $A P, A Q, Q C, C R$. The outstanding side is $P R$ in which $P B$ is, by construction, equal to $B R$. We have therefore to make $A P$ equal to twice $P B$ to make all five sides equal.

For this to be so we find that the angle $P A B$ must be (to the nearest minute) $20^{\circ} 42^{\prime}$ and thence we find that the angles $A P R, P R C$ are each $\mathrm{I} 14^{\circ} \mathrm{I} 8^{\prime}$ and the angle $A Q C \mathrm{I}_{3} \mathrm{I}^{\circ} 24^{\prime}$.

Laying off the angle $P A B$ with mathematical instruments the pentagon is constructed.

This however is not necessary because we can readily make the construction with ruler and compasses only.

On a larger scale but with corresponding letters at various points, let $R B P$ in fig. $\mathrm{r} 27$ be a side of the required pentagon, $B$ its middle point, $B Q$ a perpendicular and $B A$ drawn so as to make an angle of $45^{\circ}$ with $B P$.

With centre $P$ and radius $P R$ strike a circle to cut $B A$ at $A$.

Then $P A$ is a second side of the pentagon.

Similarly find the corresponding point $C$ by the same construction to the left of $B ; C R$ is a third side of the pentagon. 
With centre $A$ and radius $P R$ strike a circle cutting $B Q$ in $Q$.

The remaining two sides are $A Q, Q C$ and the construction is complete.

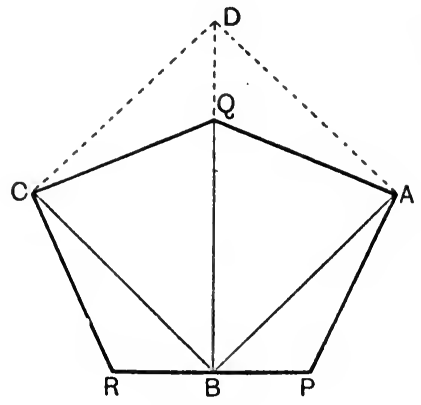

Fig. 127.

If the depicted pentagonal tessellation be studied it will be found to consist of a series of oblong hexagons in direction $\nearrow$ cut by a second similar series in direction $₫$. This observation supplies the simplest mode of construction of the tessellation.

The area of this hay-stack shaped pentagon is equal to the square upon $A B$. If we produce $B Q$ to $D$ so that $Q D=R B$ (half the length of the side of the pentagon) the figure $A B C D$ is the square upon $A B$.

This is very evident directly it is noticed that the triangles $C R B, A P B$ are equal and similar to the triangles $C Q D, A Q D$ respectively.

Of 4-sided figures, besides the square we may conveniently take as bases the various forms assumed by the rhombus and rectangle. The reader should have no difficulty in dealing with these upon the principles set forth above. In the case of the rectangle he must observe that sides of unequal lengths must be associated with different colours when designing repeating patterns.

We may adopt the equilateral pentagon as a base upon which to construct a system of repeating patterns. 


\section{THE PENTAGON BASE}

Some indeed there have been, of a more heroical strain, who striving to gaincope these ambages by venturing on a new discovery, have made their voyage in half the time.

Jон. Roвотнам to the Reader in Comenius's Janua Ling. Ed. 1659.

To take out other works in a new sampler.

MidDLETON.

When the pentagon is assembled (see fig. r 28 ) the contact system, as the reader will see at once on trial, is I to I, 2 to 3,4 to 5 .
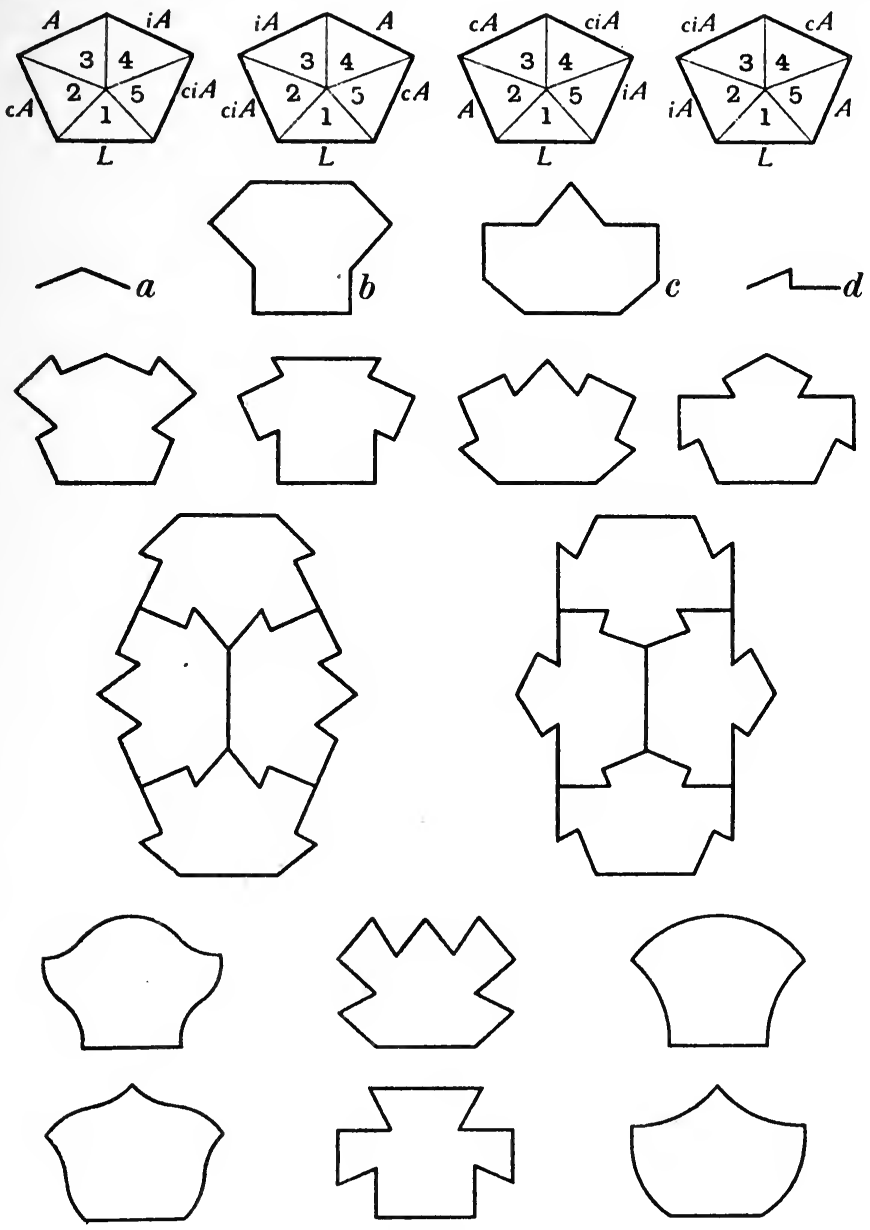

Fig. I28. 
The side being 20 millimetres, the altitude is $26.5 \mathrm{~mm}$. and the point upon the central axis to which the angular points are joined is $11 \mathrm{~mm}$. from the base.

The five compartments thus formed have no angle less than $45^{\circ}$.

The labelling of the sides shews, for any appropriate boundary $A$, four distinct symmetrical patterns.

The sides labelled $A, i A$ in the first figure are inclined to the horizontal line at the same angle that the sides labelled $c A, c i A$ are inclined to the vertical.

The angle in question is approximately $24^{\circ} \mathrm{I} 8^{\prime}$.

This fact leads us to take for $A$ in the first place the boundary as in fig. $128 \alpha$; easily constructed because the tangent of $24^{\circ} 18^{\prime}$ is '45. The base angles of the boundary are thus each $24^{\circ}$ i $8^{\prime}$.

The four resulting patterns degenerate to two, as in fig. $128 \mathrm{~b}$ and $c$, because $A=i A$ in this special case.

Next take for $A$ the form fig. I $28 d$ and we obtain the full number of patterns as shewn in the third row.

So to their work they sit, and each doth chuse

What story she will for her tapet take.

SPENSER, Muiopotmos.

I went alone to take one of all the other fragrant flowers that diapred this valley.

GREenE's Quip for an lpstart Courtier, B. 2.

\section{THE REGULAR HEXAGON BASE}

82. Numbering the compartments, as before, as in fig. I $29 a$, there are apparently only three possible arrangements. One is shewn in fig. $129 b$ in which compartments $1,2,4,5$ are adjacent

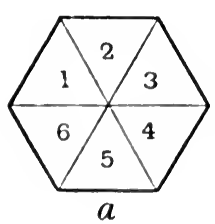

$a$

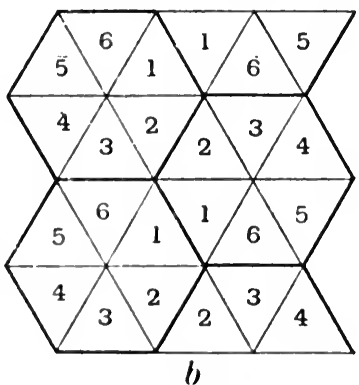

Fig. 129.

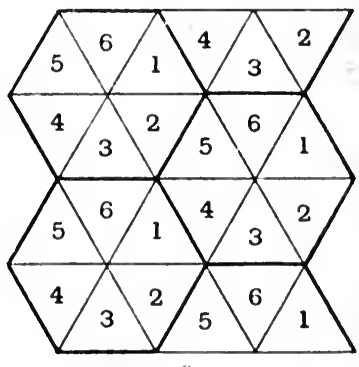

c 
to $I, 2,4,5$ respectively and 3 adjacent to 6 The piece has two aspects.

A second is shewn in fig. $129 c$ according to the contact system I to 4,2 to 5,3 to 6 . The piece has one aspect.

In these systems numbers which are not linked with different numbers may be made identical to any extent ; also two numbers linked together may be made identical and numbered at pleasure without interfering with the assemblage. The particular cases are too numerous to be separately examined in this book.

The patterns and assemblages which are shewn in fig. I 30 belong to the first of these contact systems. The assemblages exhibit each two aspects.

Nicophanes gave his mind wholly to antique pictures, partly to exemplify and take out their patterns.

Holland's Pliny.

The patterns and assemblages which are shewn in fig. I $3 \mathrm{I}$ belong to the second of these contact systems. The assemblages have each one aspect.

Take me this work out.

Othello, III. iv.

83. When the contact system is I to I, 2 to 2,3 to 6,4 to 4 , 5 to 5 the symmetry may be either about an axis perpendicular or parallel to a side. In the former case, we have for one boundary form of the first kind the set shewn in fig. I $32 a, b, c, d$.

Taking for $A$ the boundary as in fig. $132 e$ and for $B$ as in fig. $132 f$ we find the patterns given in fig. $132 g, h, k, l$.

If the symmetry be about a diagonal we have the cases of fig. I $32 m, n, o, p$.

Employing two boundary forms of the first kind $B, C$ we have fig. I $32 q$ and seven others by replacing $B$ and $C$ by $B, i C$; $i B, C ; i B, i C ; C, B ; C, i B ; i C, B ; i C, i B$ respectively.

We also have fig. $132 \mathrm{r}$ and three others by replacing $B$ and $C$ by $B, i C ; i B, C ; i B, i C$ respectively.

84. When the contact system is I to 4,2 to 5,3 to 6 we have symmetrical arrangements when the axis of symmetry is perpendicular to a side and when parallel to a side. Thus we have fig. I $33 a$ and three others obtained by replacing $B$ by $c B$, $i B, c i b$. WVe also have fig. $133 b$ and $c$ for the diagonal symmetry. 

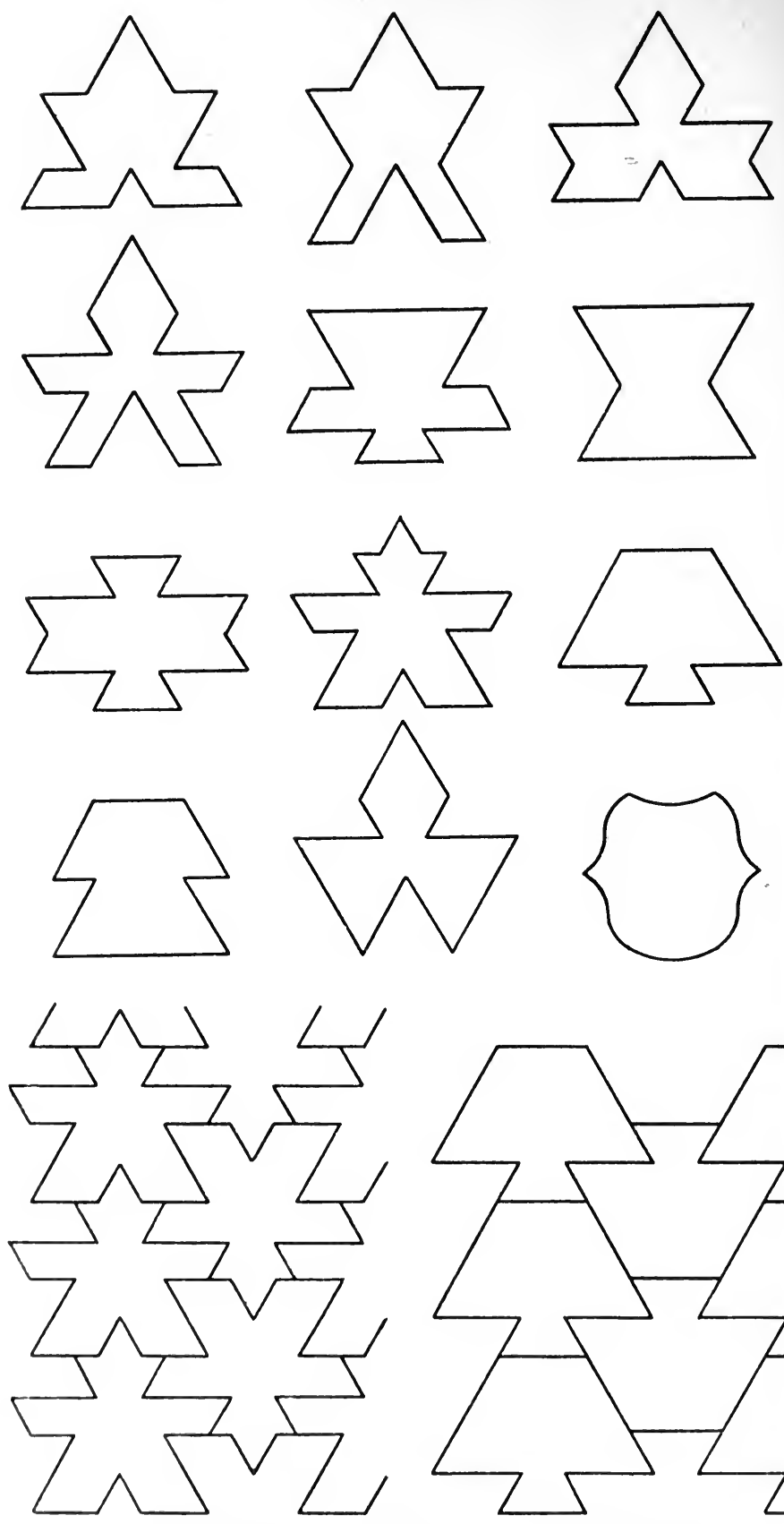

Two aspects

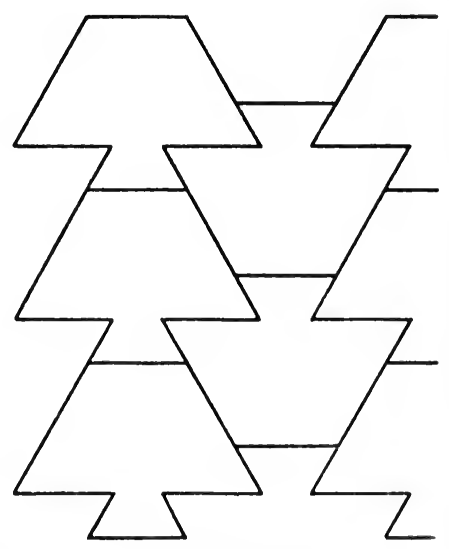

Fig. 130.

Two aspects 

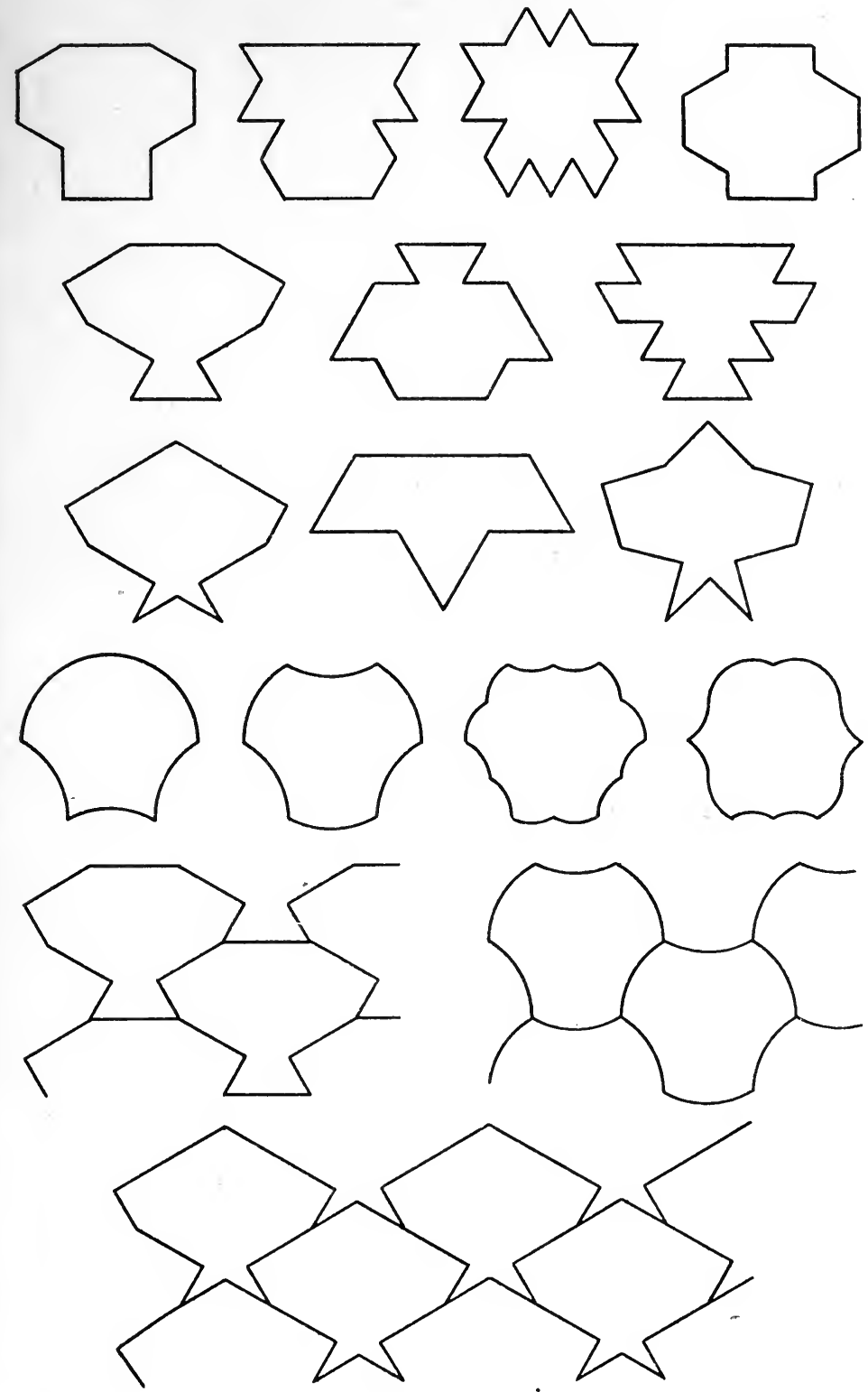

One aspect

Fig. I3I. 

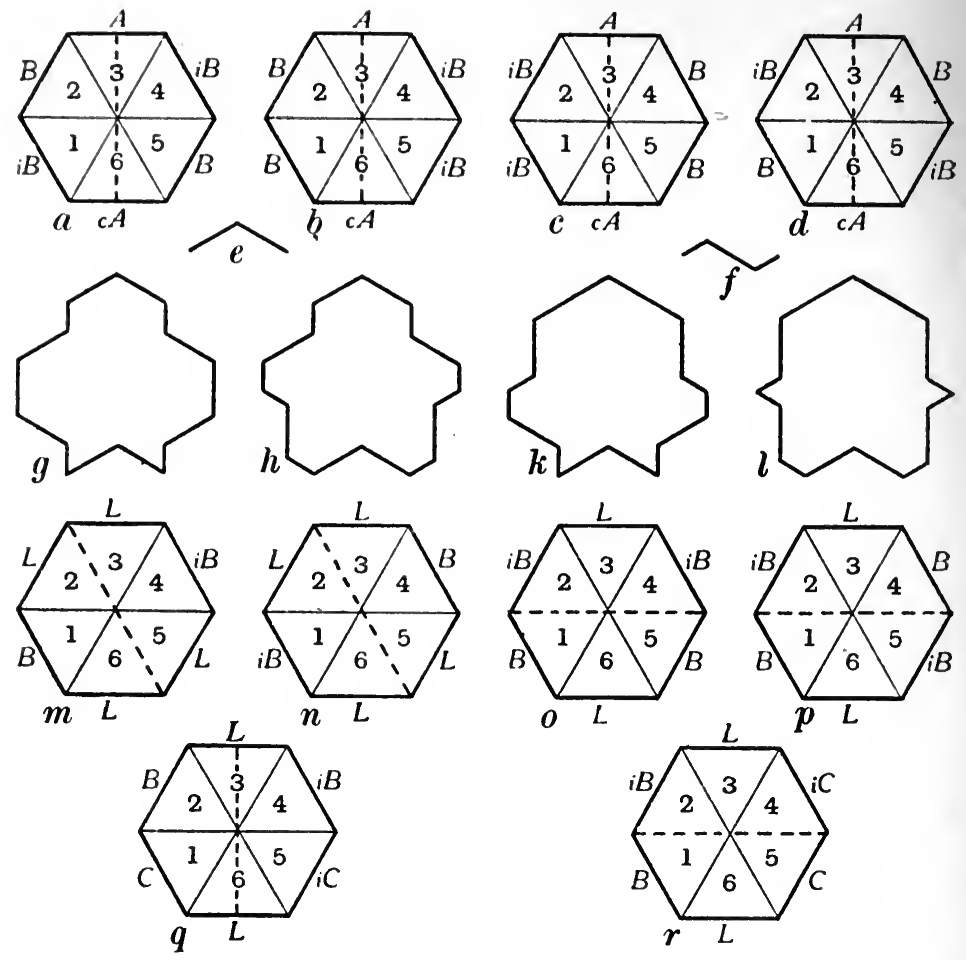

Fig. 132.

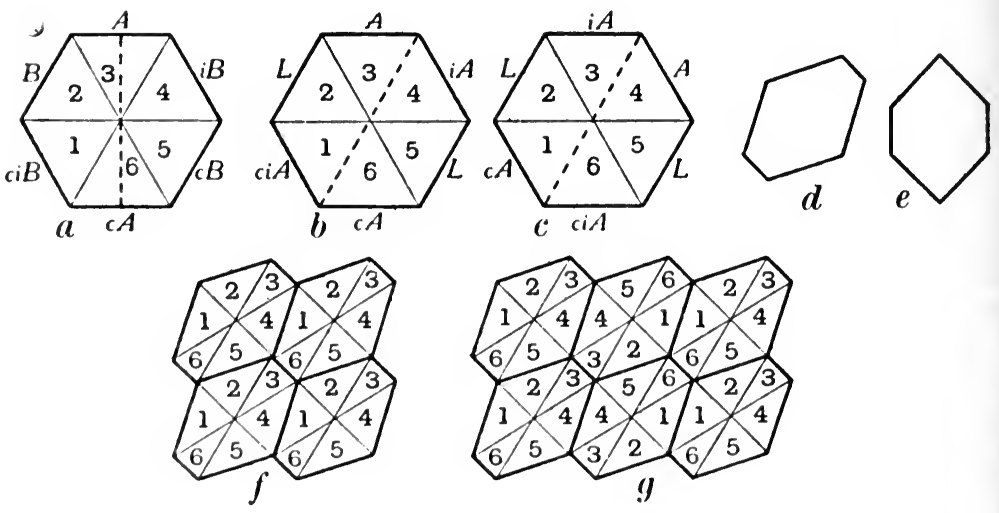

Fig. 133. 
$L$ is suitable because it is the simplest form of the second kind which is both its own complement and its own inverse.

Other hexagons may be taken as bases; such for instance as arise from the square base and are shewn in fig. $133 d$ and $e$ giving assemblages as in fig. $133 f$ ( $I$ to 4,2 to 5,3 to 6 ) and as in fig. $133 g$ ( $\mathrm{I}$ to I, 2 to 5,3 to 3,4 to 4,6 to 6 ). As with the regular hexagon two contact systems are available.

The third contact system I to 2,3 to 4,5 to 6 is established by the diagram shewn in fig. I $34 a$, which exhibits three aspects.
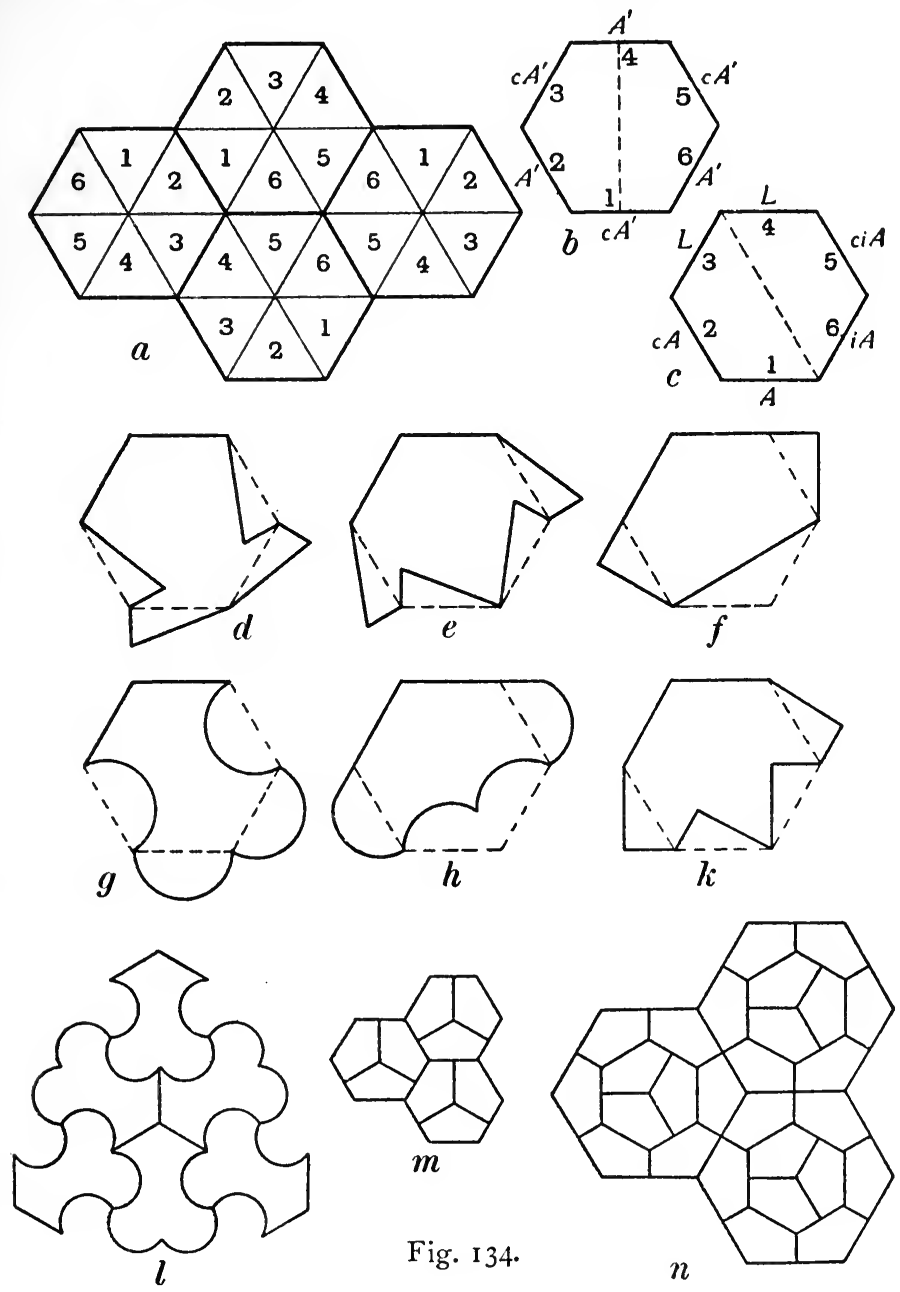
Symmetry of pattern can be obtained either about a line bisecting opposite sides or about a line bisecting opposite angles as in fig. $134 b$ and $c$ respectively.

In the first diagram $A^{\prime}$ is a self-inverse boundary.

In the second diagram $A$ is any boundary.

$L$ as usual is the unaltered straight line.

The first of these does not require attention because it is derivable from the contact system I to 4,2 to 5,3 to 6 which has been already considered.

But giving $A$ various forms, as in fig. I $34 d$ to $k$, in the second we find assemblages of which those shewn in fig. I $34 l, m, n$ are examples.

Exceptionally the pentagon pattern, which appears, can be assembled so as to exhibit either three (fig. $134 \mathrm{~m}$ ) or six aspects (fig. I $34 n$ ).

It will be noticed that the pentagon is a repeating pattern on the principle given early in this Part-that if a repeating pattern can be dissected into a number of parts of the same size and shape, the shape involved is also a repeating pattern. Here the regular hexagon is divisible into either 3 or 9 similar and equal pentagons, the pentagon being the one before us.

85. The equilateral triangle, the square, and the regular hexagon are satisfactory bases for the construction of repeating patterns because they are themselves repeating patterns. In fact any repeating pattern may be taken as a basis for the evolution of other repeating patterns. This general principle will occasionally and exceptionally be found of interest and importance.

In order to throw some light upon this development we will consider a very well known repeating pattern for linoleum, pavements, etc., viz. the combination of the regular octagon and the square (fig. $135 a$ ).

How can we obtain this form upon the principles that have been set forth?

When, as in this case, we are given a repeating pattern and we wish to determine the base and contact system to which it appertains, we have a problem which sometimes requires cleverness and ingenuity for its solution.

Take a square base as in fig. $135 b$ and the contact system 1 to 3,2 to 4 with the boundaries as in fig. $135 \mathrm{c}$. 
The result is the repeating pattern of fig. I $35 d$.

Numbering its compartments, take it as a new base as in fig. I $35 e$ with the contact system I to 4,2 to 5,3 to 6 and with the boundaries as in fig. $135 f$.
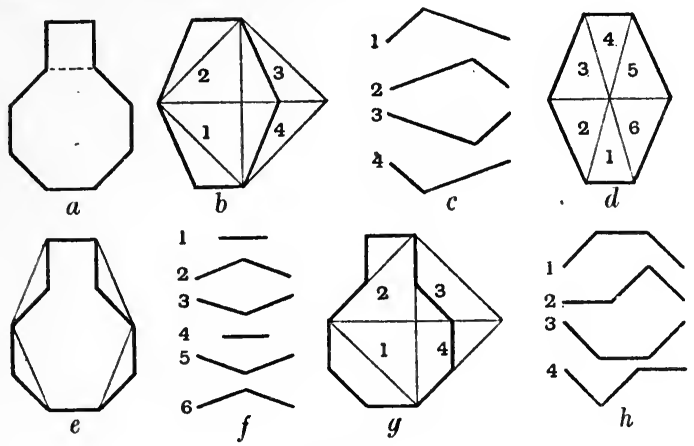

Fig. I 35 .

The result is the repeating pattern (fig. I $35 a$ ) of which we are in search. It has emerged by combining a square base and the contact system I to 3,2 to 4 with an hexagonal base having the contact system I to 4,2 to 5,3 to 6 .

We now find that the pattern may be made to emerge at once from the square base as in fig. $135 \mathrm{~g}$ with the contact system I to 3,2 to 4 by taking the boundaries as in fig. I $35 h$.

In colouring assemblages of repeating patterns it is a useful rule to employ at least as many colours as the pattern has aspects.

Usually each aspect would be associated with a separate colour, but this need not be the invariable rule.

\section{THE CONSTRUCTION OF THE PASTIMES}

Thou art a three-pil'd piece, I'll warrant thee.

Meas. for Meas. I. ii.

86. In Part I the triangles may be made at home out of stout cardboard and have a side two-and-a-half or three inches in length. The compartments should be coloured with good water-colours or oils. Four suitable colours are black, white, red and blue, as they are readily distinguishable at night. A more permanent set should be made of good dense wood. The writer's set has a three-inch side and the thickness is one-fifth of an inch. 
Oil colours are used and for the five-colour set the colours are black, white, red, olive green and dark orange. If made at home, in cardboard, pains should be taken to make the shape as accurate as possible. The pleasure of handling the pieces is much increased when they fit accurately. The ideal pieces would be as heavy as ordinary dominoes. The heavier they are the better.

The square pieces may be of one-and-a-half or two inches side and one-sixth or one-fifth of an inch thick.

It is desirable to have boards upon which to assemble the pieces, boards with a rim against which the boundary pieces may be placed. For the equilateral triangular pieces, assuming a piece of three inches side, the board should be a regular hexagon of slightly over six inches side so that the pieces will just fit in comfortably.

For the 20-set 5-colour triangular pieces the board should be the figure-of-eight shape as depicted. For the square pieces the only board required is the $6 \times 4$ board and for the right-angled triangles the shape of the hexagon used.

The irregularly shaped pieces of Part II and the designing of Part III will be much facilitated by the use of squared millimetre paper. This is preferable to squared paper in subdivisions of the inch because, at any rate in this country, it appears to be of more reliable accuracy.

The pieces should be set up with some simple boundary and then drawn on the millimetre paper. This can then be pasted upon 3-ply or other suitable wood and handed over to some one with a fret-saw to cut out. The pattern-maker should be called in if sets of great accuracy are required, and they should be as heavy as possible. 


\section{BI BLIOGRAPHY}

Bachet De Mesiriac. Problemes plaisans et delectables qui se font par les nombres. I6I2. A. Labosne. Paris, I884.

Leuvechon, JeAN. Récréation mathématique, etc. I624.

LEAKE. Mathematical Recreations. London, 1653.

OUghtred, William. Mathematical Recreations. London, I674.

Ozanan, JacQUes. Récréations mathématiques, I694. English translation

by Charles Hutton. London, I8I4.

Berckenhamp, J. A. Les amusements math. Paris, 1749.

HOOPER, W. Rational Recreations. London, 1774.

Allizeau, M. A. Les métamorphoses ou amusements géométriques. Paris, 18 I8.

JACKSON. Rational Amusements for winter evenings. London, I824.

Hugonlin. Première collection de récréations mathématiques. Paris, 1828.

Robinson, N. H. Mathematical Recreations. Albany, i851.

Lecot, V. Récréations math. Paris, i 853.

La Garrigne, F. Curiosités math. Clichy, I874.

CANTOR, M. Zahlentheoretische Spielerei. Zeitschr. für Math. I875.

Tissandikr, Gaston. Les Récréations math. ou l'enseignement par les jeux. Paris, I88I.

LUCAS, ED. Récréations math. Paris, 1882-1894.

Héraud, A. Jeux et récréations scientifiques. Paris, 1884-1903.

Rivelly, AlF. I giuochi matematici. Napoli, 1887.

LUCAS, ED. Jeux scientifiques pour servir à l'histoire, à l'enseignement et à la pratique du calcul et du dessin. Paris, s 889.

Levy, Lucien. Sur les pavages à l'aide de polygones réguliers. Bull. Soc. philomath. I 890 .

LUCAS, ED. Théories des nombres. Paris, 189I.

BAll, W. W. Rouse. Math. Recreations and Problems. 5th ed., I9II.

Sundara, Row. T. Geometrical Exercises in Paper Folding. Madras, I893.

VINOT, J. Récréations math. Paris, 1893.

LUCAS, ED. L'arithmétique amusante. Paris, 1895.

SChubert, H. Math. Mussestunden. Leipzig, I 898.

Fourrey, E. Récréations math. Paris, 1899.

Ahrens, W. Math. Unterhaltungen und Spiele. Leipzig, I90r.

Ignatiev, E. J. Math. Spiele, Rätsel und Erholungen. Petersburg, I903.

TeyssonneaU, E. Cent récréations math.; curiosités scientifiques. Paris, 1904.

Fourrey, E. Curiosités géométriques. Paris, 1907.

Dudeney, H. E. The World's Best Puzzles. Strand Magazine. IgoS. 
DUdeney, H. E. The Canterbury Puzzles. 1908.

Ahrens, W. Mathematische Unterhaltungen und Spiele. Leipzig. Aufl. I9I0; II, I9I8.

ERNST, E. Math. Unterhaltungen. Ravensburg, I9II-I2.

Ghersi, Itai.o. Matematica dilettevole e curiosa. Milano, 19I3.

GenaU, A. Math. Überraschungen. Arnsberg,1913.

Dudeney, H. E. Amusements in Mathematics. London and New York, 1917.

AHRENS, W. Altes und Neues aus der Unterhaltungsmathematik. Berlin, 19 I 8.

And now he has pour'd out his ydle mind In dainty delices and lavish joys.

SPENSER, F. Q. 11. v. 28.

P'RINTED IN ENGLAND BY J. B. PEACE, M.A. AT THF, CAMBRHIGE, UNIVERSITY PRESS 


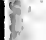

, 

PLEASE do NOT REMOVE

CARDS OR SLIPS FROM THIS POCKET

UNIVERSITY OF TORONTO LIBRARY 
